\title{
Estruturas de Poisson Não Comutativas
}

\author{
Marcos Alexandre Laudelino Orseli
}

\author{
DisSERTAÇÃo APRESENTADA \\ Instituto de MATEMÁticA E Estatística \\ Universidade DE SÃo PaUlo \\ PARA
OBTENÇÁO DO TÍTULO \\ Mestre EM CiÊnCIAS
}

Programa: Matemática

Orientador: Prof. Dr. Cristián Ortiz

Durante o desenvolvimento deste trabalho o autor recebeu auxílio financeiro CNPQ, processo: $132975 / 2017-3$

Sâo Paulo, Fevereiro de 2019 



\section{Estruturas de Poisson Não Comutativas}

Esta é a versão original da dissertação elaborada pelo candidato Marcos Alexandre Laudelino Orseli, tal como submetida à Comissão Julgadora. 



\section{Agradecimentos}

Agradeço ao CNPQ pelo financiamento e a todos que tornaram possível a produção desta dissertação. 



\section{Resumo}

Introduzimos o conceito de estrutura de Poisson não comutativa em álgebras associativas e mostra como este conceito se relaciona com o caso clássico, quando a álgebra em questão é a álgebra de funções em uma variedade de Poisson. Mostramos como quocientes simpléticos, não necessariamente suaves, fornecem exemplos de estruturas de Poisson não comutativas.

Palavras-chave: Cohomologia de Hochschild. Geometria de Poisson. Geometria Não Comutativa. 



\section{Abstract}

We introduce the concept of noncommutative Poisson structure on associative algebras and shows how this concept is related to the classical case, that is, the algebra under study is the algebra of functions on a Poisson manifold. We also show how symplectic quotients, not necessarily smooth, provides examples of noncommutative Poisson structures.

Keywords: Hochschild Cohomology. Poisson Geometry. Noncommutative Geometry. 



\section{Sumário}

$\begin{array}{lll}0 & \text { Introdução } & 7\end{array}$

1 Geometria de Poisson $\quad 9$

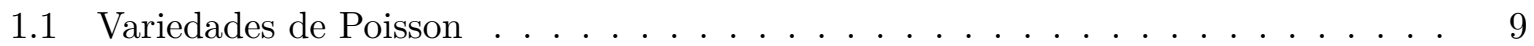

1.2 Bivetor de Poisson . . . . . . . . . . . . . . . . . . . . . 11

L.3 Colchete de Schouten . . . . . . . . . . . . . . . . . . . . . . 13

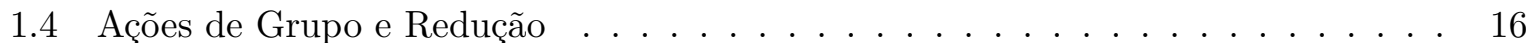

1.5 Cohomologia de Poisson $\ldots \ldots \ldots \ldots \ldots \ldots$

1.6 Cohomologia de Poisson e Cohomologia de de Rham . . . . . . . . . . . . . 19

2 Geometria Não Comutativa 20

2.1 Grupoides . . . . . . . . . . . . . . . . . . . . . . . . . . . 20

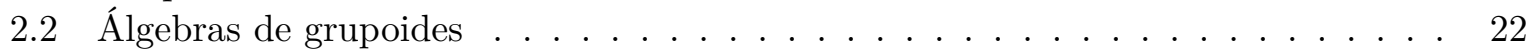

3 Cohomologia de Hochschild 26

3.1 Definição de Homologia e Cohomologia de Hochschild . . . . . . . . . . . 26

3.2 Interpretação em Graus Baixos $\ldots \ldots \ldots$. . . . . . . . . . . . . . 30

3.3 Produto Cup . . . . . . . . . . . . . . . . . . . . . . . 31

3.4 Colchete de Gerstenhaber . . . . . . . . . . . . . . . . . . . . 31

3.5 versão Topológica . . . . . . . . . . . . . . . . . . . . . . . . . . . 32

3.6 Equivalência de Morita . . . . . . . . . . . . . . . . . . . . . . . . . . . 34

3.7 Hochschild-Kostant-Rosenberg $\ldots \ldots \ldots \ldots \ldots \ldots \ldots$

4 Geometria de Poisson Não Comutativa $\quad 39$

4.1 Estruturas de Poisson em Álgebras . . . . . . . . . . . . . . . . . . . . . . . . 39

4.2 Cohomologia de Poisson . . . . . . . . . . . . . . . . . . . . . . . 40

4.3 Redução de Poisson $\ldots \ldots \ldots \ldots$. . . . . . . . . . . . . . . . . . . 42

4.4 Toro näo comutativo . . . . . . . . . . . . . . . . . . . . 46

4.5 Outras Aplicaçóes $\ldots \ldots \ldots \ldots$. . . . . . . . . . . . . . . . . . 53

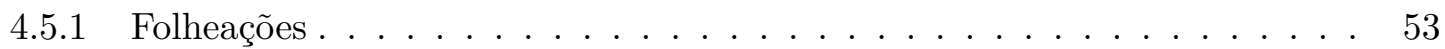

4.52 Orbifolds . . . . . . . . . . . . . . . . . . . 54

4.5 .3 Dinâmica Hamiltoniang . . . . . . . . . . . . . . . . . 54

$\begin{array}{ll}\text { Referências Bibliográficas } & 55\end{array}$ 



\section{Capítulo 0}

\section{Introdução}

Em Geometria Diferencial os objetos fundamentais de estudo são as variedades diferenciáveis. Muitas vezes, uma variedade $M$ vem munida de uma noção de simetria a qual determina uma relação de equivalência $\mathcal{R} \subseteq M \times M$. Por exemplo, a relação de equivalência pode ser dada pela ação de um grupo de Lie ou pelas folhas de uma folheação regular. Neste contexto, o problema principal consiste em estudar o espaço topológico quociente $M / \sim$ definido pela relação de equivalência $\mathcal{R} \subseteq M \times M$. Este espaço quociente pode ser suave, mas em geral isto não acontece e o espaço quociente $M / \sim$ pode ter singularidades, e.g. orbifolds. O Critério de Godement dá condições para que um quociente $M / \sim$ seja suave. De forma precisa, se $\mathcal{R} \subseteq M \times M$ é uma subvariedade fechada e as projeções $p r_{1}, p r_{2}: \mathcal{R} \subseteq M \times M \rightarrow M$ são submersões sobrejetoras, então $M / \sim$ herda uma estrutura de variedade diferenciável carcaterizada pela projeção $M \rightarrow M / \sim$ ser uma submersão sobrejetora. Decorre deste resultado que se $G \times M \rightarrow M$ é uma ação livre e própria, então o espaço de órbitas $M / G$ é uma variedade suave.

O critério de Godement pode ser interpretado em termos de grupoides de Lie. Um grupoide de Lie é um grupoide interno na categoria das variedades diferenciáveis. Toda submersão sobrejetora $p: M \rightarrow N$ determina o grupoide de submersão $M \times_{N} M$ sobre $M$, que nada mais é do que o gráfico da relação de equivalência em $M$ dada por $x \sim y$ se $p(x)=p(y)$. Todo grupoide de Lie tem um espaço de órbitas, o qual se reduz a $N$ no caso do grupoide de submersão $M \times_{N} M$. O critério de Godement de fato diz que todo quociente suave de uma variedade diferenciável é realizado como o espaço de órbitas de um grupoide de submersão.

Grupoides de Lie unificam várias estruturas, incluindo submersões, grupos de Lie, variedades, ações de grupos de Lie, folheações, entre outras. Em cada caso, temos um espaço de órbitas que pode não ser suave. Para lidarmos com estes quocientes não suaves, nesta dissertação usamos ideias da Geometria não comutativa. A ideia é substituir a álgebra de funções no quociente por uma álgebra associativa canonicamente associada, tipicamente a álgebra de convolução de um grupoide.

Outro problema que aparece no estudo de simetrias em variedades é o problema da redução de estruturas geométricas. Por exemplo, em geometria simplética muitas vezes precisamos estudar reduções simpléticas e reduções de Poisson, onde é essencial que a ação que temos na variedade seja livre e própria para que os quocientes façam sentido como variedades simpléticas ou de Poisson. De forma precisa, se $(M, \pi)$ é uma variedade de Poisson e $G$ é um grupo de Lie que age em $M$ preservando a estrutura de Poisson, então a álgebra de funções invariantes $C^{\infty}(M)^{G} \subseteq$ $C^{\infty}(M)$ é uma subálgebra de Poisson. Em particular, se a ação é livre e própria, a variedade quociente $M / G$ herda uma estrutura de Poisson através da identificação $C^{\infty}(M)^{G} \cong C^{\infty}(M / G)$.

Porém as vezes precisamos encarar a situação em que os quocientes usuais no processo de redução não são suaves e gostaríamos de entender em que sentido o quociente $M / G$ admite uma estrutura de Poisson. Esta pergunta é a motivação principal da presente dissertação, na qual estudamos o conceito de estrutura de Poisson não comutativa, i.e. uma estrutura de Poisson em uma álgebra não necessariamente comutativa. Veremos que um quociente não suave $M / G$ de 
uma variedade de Poisson pode ser pensado como uma estrutura de Poisson não comutativa em uma álgebra produto cruzado.

O conceito de estrutura de Poisson não comutativa foi introduzido por Xu ( $\mathrm{X} u$, [1994) e estudado por outros autores ((Halbout \& Tang, 2010) (Tang, 2004), (Block \& Getzler, 1992)), (Kordyukov, [2010)). A ideia central é a seguinte: uma versão do Teorema de Hochschild-Kostant-Rosenberg B.7.] estabelece um isomorfismo

$$
H H^{*}\left(C^{\infty}(M)\right) \cong \Gamma^{\infty}\left(\Lambda^{*} T M\right),
$$

entre a cohomologia de Hochschild da álgebra de funções $C^{\infty}(M)$ em uma variedade $M$ e o espaço dos multivetores em $M$. Em particular, uma estrutura de Poisson $\pi \in \Gamma\left(\Lambda^{2} T M\right)$ corresponde a um 2-cociclo de Hochschild $\tilde{\pi}$ em $C^{\infty}(M)$ tal que o colchete de Gerstenhaber $\llbracket \tilde{\pi}, \tilde{\pi} \rrbracket$ é um 3cobordo de Hochschild. Desta forma, se $A$ é uma álgebra associativa qualquer, uma estrutura de Poisson não comutativa em $A$ é definida por um 2-cociclo de Hochschild $\tilde{\pi}$ em $A$ tal que o colchete de Gerstenhaber $\llbracket \tilde{\pi}, \tilde{\pi} \rrbracket=0$ é zero na cohomologia de Hochschild de grau 3.

Nesta dissertação estudamos estruturas de Poisson não comutativas e apresentamos as principais propriedades e exemplos. A dissertação esta organizada da seguinte forma. No Capítulo 1 apresentamos os conceitos básicos da Geometria de Poisson, incluindo bivetores de Poisson, exemplos principais bem como a definição da cohomologia de Poisson de uma variedade de Poisson. No capítulo 2, discutimos brevemente como a álgebras de convolução de um grupoides serve como modelo para a álgebra de funções de um espaço singular. O Capítulo 3 apresenta de forma detalhada o complexo de Hochschild de uma álgebra associativa, bem como a descrição da cohomologia de Hochschild em termos de funtores derivados. Também é introduzido o colchete de Gerstenhaber e a prova de uma versão do Teorema de Hochschild-Kostant-Rosenberg. O Capítulo 4 introduz o conceito de estrutura de Poisson não comutativa e representa a parte principal deste trabalho. Mostramos como um quociente de uma variedade de Poisson determina uma estrutura de Poisson não comutativa em uma álgebra produto cruzado (Teorema 4.3.3). Também provamos que o toro não comutativo possui uma estrutura de Poisson não comutativa natural (Teorema 4.4.2). Encerramos o trabalho apresentando brevemente outros resultados existentes em geometria de Poisson não comutativa. 


\section{Capítulo 1}

\section{Geometria de Poisson}

\subsection{Variedades de Poisson}

Em mecânica clássica o estado de um sistema com $n$ graus de liberdade no tempo $t$ é dado por um ponto $\left(q_{i}(t), p_{i}(t)\right), i=1, \ldots, n$ em $\mathbf{R}^{2 n}$, onde $q_{i}$ são chamadas coordenadas posição, $p_{i}$ coordenadas momento e $\mathbf{R}^{2 n}$ é chamado espaço de fase do sistema. Temos que a evolução do sistema no tempo é governada por uma função $H: \mathbf{R}^{2 n} \rightarrow \mathbf{R}$ chamada Hamiltoniano no sentido de que o sistema satisfaz as equações de Hamilton:

$$
\left\{\begin{array}{l}
\dot{q}_{i}=\frac{\partial H}{\partial p_{i}} \\
\dot{p}_{i}=-\frac{\partial H}{\partial q_{i}}
\end{array} \quad(i=1, \ldots, n)\right.
$$

Definindo o colchete

$$
\{f, g\}:=\sum_{i=1}^{n} \frac{\partial f}{\partial p_{i}} \frac{\partial g}{\partial q_{i}}-\frac{\partial f}{\partial q_{i}} \frac{\partial g}{\partial p_{i}},
$$

vemos que dado um Hamiltoniano $H: \mathbb{R}^{2 n} \rightarrow \mathbb{R}$ as equações de Hamilton são dadas por:

$$
\dot{x}_{i}=\left\{H, x_{i}\right\}, \quad(i=1, \ldots, n)
$$

onde $x_{i}$ é uma das coordenadas $p_{i}$ ou $q_{i}$.

Este colchete possui as seguintes propriedades:

1. Antissimetria: $\{f, g\}=-\{g, f\}$

2. $\mathbb{R}$-bilinearidade: $\{f, a g+b h\}=a\{f, g\}+b\{f, h\}$

3. Identidade de Jacobi: $\{f,\{g, h\}\}+\{g,\{h, f\}\}+\{h,\{f, g\}\}=0$

4. Identidade de Leibniz: $\{f, g h\}=g\{f, h\}+\{f, g\} h$

Note que a identidade de Leibniz nos dá que $\{H, \cdot\}$ é uma derivação ou seja, existe um campo de vetores $X_{H}$ tal que

$$
X_{H}(f)=\{H, f\},
$$

tal campo é chamado de campo Hamiltoniano associado a $H$. Observe que o campo Hamiltoniano é escrito em coordenadas da seguinte maneira:

$$
X_{H}=\sum_{i=1}^{n} \frac{\partial H}{\partial p_{i}} \frac{\partial}{\partial q_{i}}-\frac{\partial H}{\partial q_{i}} \frac{\partial}{\partial p_{i}}
$$

logo as equações de Hamilton são apenas as equações das curvas integrais de $X_{H}$. Isto é,

$$
\dot{x}(t)=X_{H}(x(t)) \text {. }
$$


Desta forma nós escrevemos as equações de Hamilton de uma forma geométrica, dependendo apenas do colchete. O fato dos espaços de fase não serem sempre espaços euclidianos motiva a seguinte definição:

Definição 1.1.1. Um colchete de Poisson em uma variedade $M$ é uma aplicação

$$
\{\cdot, \cdot\}: C^{\infty}(M) \times C^{\infty}(M) \rightarrow C^{\infty}(M)
$$

que satisfaz:

1. Antissimetria: $\{f, g\}=-\{g, f\}$

2. $\mathbb{R}$-bilinearidade: $\{f, a g+b h\}=a\{f, g\}+b\{f, h\}$

3. Identidade de Jacobi: $\{f,\{g, h\}\}+\{g,\{h, f\}\}+\{h,\{f, g\}\}=0$

4. Identidade de Leibniz: $\{f, g h\}=g\{f, h\}+\{f, g\} h$

para $f, g, h \in C^{\infty}(M)$.

Uma variedade de Poisson $(M,\{\cdot, \cdot\})$ é uma variedade suave $M$ com um colchete de Poisson em $C^{\infty}(M)$.

Exemplo 1.1.1. $\mathbb{R}^{2 n}$ com o colchete definido anteriormente é uma variedade de Poisson.

Exemplo 1.1.2. Toda variedade suave possui um colchete de Poisson trivial definido por $\{f, g\}=0, \forall f, g \in C^{\infty}(M)$.

Exemplo 1.1.3. O espaço dual de uma álgebra de Lie de dimensão finita possui uma estrutura de Poisson canônica chamada estrutura de Lie-Poisson. Primeiramente observe que se $\mathfrak{g}^{*}$ é o espaço dual de uma álgebra de Lie $\mathfrak{g}$, podemos identificar o espaço tangente em cada ponto de $\mathfrak{g}^{*}$ com $\mathfrak{g}^{*}$. Assim, dada $f \in C^{\infty}\left(\mathfrak{g}^{*}\right)$ temos que

$$
\mathrm{d} f_{\alpha}: T_{\alpha} \mathfrak{g}^{*}=\mathfrak{g}^{*} \rightarrow \mathbb{R}
$$

pode ser identificada com um elemento de $\mathfrak{g}$. Logo definimos o colchete de Poisson em $\mathfrak{g}^{*}$ da seguinte forma

$$
\begin{aligned}
\{f, g\}: \mathfrak{g}^{*} & \rightarrow \mathbb{R} \\
\theta & \mapsto-\theta[\mathrm{d} f, \mathrm{~d} g],
\end{aligned}
$$

onde $f, g \in C^{\infty}\left(\mathfrak{g}^{*}\right)$.

Exemplo 1.1.4. Suponha que $(M, \omega)$ é uma variedade simplética, isto é, uma variedade suave $M$ com uma 2-forma não degenerada $\omega$. Podemos definir uma estrutura de Poisson em $M$ da seguinte maneira

$$
\{f, g\}=\omega\left(X_{g}, X_{f}\right),
$$

onde $X_{g}$ é o campo Hamiltoniano associado a $g$, i.e, $\omega\left(X_{g}, \bullet\right)=\mathrm{d} g$.

Uma função $f \in C^{\infty}(M)$ é chamada integral primeira de um campo vetorial $X$ se

$$
\frac{d}{d t}(f(x(t)))=0
$$

para cada curva integral $x(t)$ de $X$. Observe que $f$ é integral primeira se, e somente se, $X(f)=0$. Conhecer integrais primeiras nos permite reduzir o número de variáveis no problema de encontrar as curvas integrais de um campo vetorial. Quando o campo for um campo Hamiltoniano $X_{h}$ temos que $f$ é integral primeira se, e somente se,

$$
X_{h}(f)=\{h, f\}=0
$$

disto segue imediatamente que o Hamiltoniano é uma quantidade conservada: 
Teorema 1.1.1. O Hamiltoniano $h$ é uma integral primeira do campo Hamiltoniano $X_{h}$.

Demonstração. Pela antissimetria do colchete de Poisson temos que

$$
X_{h}=\{h, h\}=0
$$

O colchete de Poisson também nos permite encontrar mais integrais primeiras:

Teorema 1.1.2. Se $f, g \in C^{\infty}(M)$ são integrais primeiras do campo Hamiltoniano $X_{h}, h \in$ $C^{\infty}(M)$, então $\{f, g\}$ também é uma integral primeira de $X_{h}$.

Demonstração. Da identidade de Jacobi segue que:

$$
X_{h}(\{f, g\})=\{h,\{f, g\}\}=\{\{g, h\}, f\}+\{\{h, f\}, g\}=0 .
$$

\subsection{Bivetor de Poisson}

Nesta seção expressaremos as estruturas de Poisson em uma variedade suave $M$ de uma forma geométrica. Isto é, em termos de tensores.

Sejam $M$ uma variedade suave e $q$ um inteiro positivo. Denotaremos por $\Lambda^{q}(T M)$ o espaço $q$-vetores em $M . \Lambda^{q}(T M)$ é um fibrado vetorial sobre $M$ cuja fibra sobre um ponto $p \in M$ é o espaço vetorial $\Lambda^{q}\left(T_{p} M\right)$ que é o produto exterior de $q$ cópias de $T_{p} M$. Se $\left(x_{1}, \ldots, x_{n}\right)$ é um sistema de coordenadas locais em $p \in M$ então os elementos $\frac{\partial}{\partial x_{i_{1}}} \wedge \cdots \wedge \frac{\partial}{\partial x_{i_{q}}}(p) \operatorname{com} i_{1}<\cdots<i_{q}$ formam uma base de $\Lambda^{q}\left(T_{p} M\right)$ e além disso, note que como $T M$ é trivial sobre o domínio de qualquer sistema de coordenadas, o fibrado $\Lambda^{q}(T M)$ também é.

Um campo suave de q-vetores em $M$ é uma seção suave de $\Lambda^{q}(T M)$. Isto é, uma função suave $\Pi: M \rightarrow \Lambda^{q}(T M)$ que satisfaz $\Pi(p) \in \Lambda^{q}\left(T_{p} M\right)$ para todo $p \in M$. Em coordenadas locais $x=\left(x_{1}, \ldots, x_{n}\right), \Pi$ tem a expressão

$$
\Pi(x)=\sum_{i_{1}<\cdots<i_{q}} \Pi_{i_{1} \ldots i_{q}}(x) \frac{\partial}{\partial x_{i_{1}}} \wedge \ldots \wedge \frac{\partial}{\partial x_{i_{q}}}
$$

onde as componentes $\Pi_{i_{1} \ldots i_{q}}$ são funções suaves que chamaremos de coeficientes de $\Pi$.

Podemos interpretar os $q$-vetores como objetos duais às $q$-formas diferenciais da seguinte maneira. Se $\Pi$ é um $q$-vetor e $\omega$ é uma $q$-forma diferencial que são expressados em coordenadas por $\Pi(x)=\sum_{i_{1}<\cdots<i_{q}} \Pi_{i_{1} \ldots i_{q}} \frac{\partial}{\partial x_{i_{1}}} \wedge \ldots \wedge \frac{\partial}{\partial x_{i_{q}}}$ e $\omega(x)=\sum_{i_{1}<\cdots<i_{q}} \omega_{i_{1} \ldots i_{q}} \mathrm{~d} x_{i_{1}} \wedge \ldots \wedge \mathrm{d} x_{i_{q}}$, onde $\Pi_{i_{1} \ldots i_{q}}$ e $\omega_{i_{1} \ldots i_{q}}$ são funções suaves, então temos um pareamento

$$
<\omega, \Pi>=\sum_{i_{1}<\cdots<i_{q}} \omega_{i_{1} \ldots i_{q}} \Pi_{i_{1} \ldots i_{q}}
$$

Usaremos também a notação $\omega(\Pi)$ para $<\omega, \Pi>$.

Observe que a definição de $\langle\omega, \Pi>$ é independente da escolha de coordenadas. De fato, se $y=\left(y_{1}, \ldots, y_{n}\right)$ é outro sistema de coordenadas no qual temos $\Pi(y)=\sum_{i_{1}<\cdots<i_{q}} \Pi_{i_{1} \ldots i_{q}}^{\prime} \frac{\partial}{\partial y_{i_{1}}} \wedge$ $\ldots \wedge \frac{\partial}{\partial y_{i_{q}}}$ e $\omega(y)=\sum_{i_{1}<\cdots<i_{q}} \omega_{i_{1} \ldots i_{q}}^{\prime} \mathrm{d} y_{i_{1}} \wedge \ldots \wedge \mathrm{d} y_{i_{q}}$ então

$$
\omega_{i_{1} \ldots i_{q}}=\omega_{i_{1} \ldots i_{q}}^{\prime} \frac{\partial\left(y_{i_{1}}, \ldots, y_{i_{q}}\right)}{\partial\left(x_{i_{1}}, \ldots, x_{i_{q}}\right)} \text { e } \Pi i_{1} \ldots i_{q}=\Pi_{i_{1} \ldots i_{q}}^{\prime} \frac{\partial\left(x_{i_{1}}, \ldots, x_{i_{q}}\right)}{\partial\left(y_{i_{1}}, \ldots, y_{i_{q}}\right)} .
$$


Portanto

$$
\begin{aligned}
\omega_{i_{1} \ldots i_{q}} \Pi i_{1} \ldots i_{q} & =\omega_{i_{1} \ldots i_{q}}^{\prime} \frac{\partial\left(y_{i_{1}}, \ldots, y_{i_{q}}\right)}{\partial\left(x_{i_{1}}, \ldots, x_{i_{q}}\right)} \Pi_{i_{1} \ldots i_{q}}^{\prime} \frac{\partial\left(x_{i_{1}}, \ldots, x_{i_{q}}\right)}{\partial\left(y_{i_{1}}, \ldots, y_{i_{q}}\right)} \\
& =\omega_{i_{1} \ldots i_{q}}^{\prime} \Pi_{i_{1} \ldots i_{q}}^{\prime} .
\end{aligned}
$$

Desta discussão segue que podemos considerar os $q$-vetores como operadores $C^{\infty}(M)$-lineares de $q$-formas diferenciais para $C^{\infty}(M)$ e as $q$-formas diferenciais como operadores $C^{\infty}(M)$-lineares de $q$-vetores para $C^{\infty}(M)$.

Assim como campos vetoriais podem ser identificados com derivações da álgebra $C^{\infty}(M)$, dado um $q$-vetor $\Pi$ podemos definir uma aplicação $\mathbb{R}$-multilinear anti-simétrica de $C^{\infty}(M) \underbrace{\times \cdots \times}_{q \text {-vezes }} C^{\infty}(M)$ em $C^{\infty}(M)$ por

$$
\Pi\left(f_{1}, \ldots, f_{q}\right):=\Pi\left(\mathrm{d} f_{1} \wedge \cdots \wedge \mathrm{d} f_{q}\right) .
$$

De modo inverso temos

Lema 1.2.1. Uma aplicação $\mathbb{R}$-multilinear $\Pi: C^{\infty}(M) \underbrace{\times \cdots \times}_{q \text {-vezes }} C^{\infty}(M) \rightarrow C^{\infty}(M)$ vem de um q-vetor pela fórmula 1.$]$ se, e somente se, $\Pi$ é anti-simétrica e satisfaz a regra de Leibniz

$$
\Pi\left(f g, f_{2}, \ldots, f_{q}\right)=f \Pi\left(g, f_{2}, \ldots, f_{q}\right)+\Pi\left(f, f_{2}, \ldots, f_{q}\right) g .
$$

Chamaremos uma aplicação que satisfaz as condições acima de multi-derivação. O que o lema acima nos diz é que podemos identificar campos de multi-vetores com multi-derivações.

Demonstração. Observe que o "somente se"segue diretamente da fórmula ㄸ. . Agora primeiramente temos que mostrar que o valor de $\Pi\left(f_{1}, \ldots, f_{q}\right)$ em um ponto $p \in M$ depende apenas do valor de $\mathrm{d} f_{1}, \ldots, \mathrm{d} f_{q}$ em $p$. Equivalentemente basta verificar que se $\mathrm{d} f_{1}(p)=0$ então $\Pi\left(f_{1}, \ldots, f_{q}\right)=0$. Se d $f_{1}(p)=0$ temos, pelo teorema de Taylor, $f_{1}=c+\sum_{i} x_{i} g_{i}$, para alguma constante $c$, onde $x_{i}$ e $g_{i}$ são funções suaves que se anulam em $p$. Pela regra de Leibniz temos

$$
\Pi\left(1.1, \ldots, f_{q}\right)=\Pi\left(1, \ldots, f_{q}\right)+\Pi\left(1, \ldots, f_{q}\right) \Rightarrow \Pi\left(1, \ldots, f_{q}\right)=0
$$

e, portanto,

$$
\begin{aligned}
\Pi\left(f_{1}, \ldots, f_{q}\right)(p) & =\Pi\left(c+\sum_{i} x_{i} g_{i}, \ldots, f_{q}\right)(p)=\Pi\left(c, \ldots, f_{q}\right)(p)+\sum_{i} \Pi\left(x_{i} g_{i}, \ldots, f_{q}\right)(p) \\
& =c \Pi\left(1, \ldots, f_{q}\right)(p)+\sum_{i} x_{i}(p) \Pi\left(g_{i}, \ldots, f_{q}\right)(p)+\sum_{i} g_{i}(p) \Pi\left(x_{i}, \ldots, f_{q}\right)(p) \\
& =0 .
\end{aligned}
$$

Logo, uma multi-derivação é um operador $C^{\infty}(M)$-linear no espaço das formas diferenciais e portanto pode ser identificada com um multi-vetor.

Um caso particular do lema anterior são os colchetes de Poisson. Como um colchete de Poisson é uma aplicação $\mathbb{R}$-multilinear $\{\cdot, \cdot\}: C^{\infty}(M) \times C^{\infty}(M) \rightarrow C^{\infty}(M)$ anti-simétrica e que satisfaz a regra de Leibniz segue do lema que

$$
\{f, g\}=\pi(\mathrm{d} f \wedge \mathrm{d} g)
$$

para algum bivetor $\pi$, onde $f, g \in C^{\infty}(M)$.

Um bivetor $\pi$ tal que o colchete $\{f, g\}:=\pi(\mathrm{d} f \wedge \mathrm{d} g)$ é um colchete de Poisson é chamado bivetor de Poisson. 
Dadas coordenadas $\left(x_{1}, \ldots, x_{n}\right)$, um bivetor de Poisson $\pi$ é dado nessas coordenadas por

$$
\pi=\sum_{i<j} \pi_{i j} \frac{\partial}{\partial x_{i}} \wedge \frac{\partial}{\partial x_{j}}=\frac{1}{2} \sum_{i, j} \pi_{i j} \frac{\partial}{\partial x_{i}} \wedge \frac{\partial}{\partial x_{j}}
$$

onde $\pi_{i j} \in C^{\infty}(M)$ é a função dada por $\pi_{i j}=\pi\left(\mathrm{d} x_{i} \wedge \mathrm{d} x_{j}\right)=\left\{x_{i}, x_{j}\right\}$, e

$$
\{f, g\}=\sum_{i<j} \pi_{i j} \frac{\partial}{\partial x_{i}} \wedge \frac{\partial}{\partial x_{j}}\left(\sum_{i, j} \frac{\partial f}{\partial x_{i}} \frac{\partial g}{\partial x_{j}} \mathrm{~d} x_{i} \wedge \mathrm{d} x_{j}\right)=\sum_{i, j} \pi_{i j} \frac{\partial f}{\partial x_{i}} \frac{\partial g}{\partial x_{j}} .
$$

\subsection{Colchete de Schouten}

Nesta seção introduziremos um colchete para multivetores que estende de forma natural o colchete de Lie de campos vetoriais. Este colchete nos dará uma condição geométrica para um bivetor ser um bivetor de Poisson.

Se $A=\sum_{i} a_{i} \frac{\partial}{\partial x_{i}}$ e $B=\sum_{i} b_{i} \frac{\partial}{\partial x_{i}}$ são dois campos de vetores escritos em um sistema de coordenadas $\left(x_{1}, \ldots, x_{n}\right)$, o colchete de Lie de $A$ e $B$ é dado por

$$
[A, B]=\sum_{i} a_{i}\left(\sum_{j} \frac{\partial b_{j}}{\partial x_{i}} \frac{\partial}{\partial x_{j}}\right)-\sum_{i} b_{i}\left(\sum_{j} \frac{\partial a_{j}}{\partial x_{i}} \frac{\partial}{\partial x_{j}}\right) .
$$

Escrevendo $\zeta_{i}=\frac{\partial}{\partial x_{i}}$ e tomando a álgebra gerada pelos $\zeta_{i}$ com as relações $\zeta_{i} \zeta_{j}=-\zeta_{j} \zeta_{i}$, podemos escrever formalmente os campos de vetores como $A=\sum_{i} a_{i} \zeta_{i}$ e $B=\sum_{i} b_{i} \zeta_{i}$ e considerá-los como funções das variáveis $\left(x_{i}, \zeta_{i}\right)$, lineares em $\zeta_{i}$. Desta forma o colchete de Lie de $A$ e $B$ se escreve como

$$
[A, B]=\sum_{i} \frac{\partial A}{\partial \zeta_{i}} \frac{\partial B}{\partial x_{i}}-\sum_{i} \frac{\partial B}{\partial \zeta_{i}} \frac{\partial A}{\partial x_{i}}
$$

$\mathrm{Se}$

$$
\Pi=\sum_{i_{1}<\cdots<i_{p}} \Pi_{i_{1} \ldots i_{p}} \frac{\partial}{\partial x^{i_{1}}} \wedge \ldots \wedge \frac{\partial}{\partial x^{i_{p}}} \in \Gamma\left(\Lambda^{p} T M\right)
$$

é um $p$-multivetor dado nas coordenadas $\left(x_{1}, \ldots, x_{n}\right)$, escrevendo nas coordenadas $\left(x_{i}, \zeta_{i}\right)$ obtemos

$$
\Pi=\sum_{i_{1}<\cdots<i_{p}} \Pi_{i_{1} \ldots i_{p}} \zeta_{i_{1}} \ldots \zeta i_{p} .
$$

Vamos utilizar a seguinte regra para derivadas nas variáveis $\zeta_{i}$ :

$$
\frac{\partial\left(\zeta_{i_{1}} \ldots \zeta_{i_{p}}\right)}{\partial \zeta_{i_{k}}}:=(-1)^{p-k} \zeta_{i_{1}} \ldots \widehat{\zeta_{i_{k}}} \ldots \zeta_{i_{p}}
$$

Se $A=\sum_{i_{1}<\cdots<i_{a}} A_{i_{1} \ldots i_{a}} \zeta_{i_{1}} \ldots \zeta i_{a}$ e $B=\sum_{i_{1}<\cdots<i_{b}} B_{i_{1} \ldots i_{b}} \zeta_{i_{1}} \ldots \zeta i_{b}$ são dois multivetores, a expressão que obtemos para o colchete de Lie nos motiva a definir

$$
[A, B]=\sum_{i} \frac{\partial A}{\partial \zeta_{i}} \frac{\partial B}{\partial x^{i}}-(-1)^{(a-1)(b-1)} \sum_{i} \frac{\partial B}{\partial \zeta_{i}} \frac{\partial A}{\partial x^{i}} .
$$

Temos que $[A, B]$ é um polimômio homogêneo nas variáveis $\zeta_{i}$ de grau $a+b-1$, e portanto $[A, B]$ é um $(a+b-1)$-multivetor.

Teorema 1.3.1. O colchete definido anteriormente possui as seguintes propriedades:

1. Anti-comutatividade graduada: se $A$ é um a-multivetor e $B$ é um b-multivetor então

$$
[A, B]=(-1)^{(a-1)(b-1)}[B, A] .
$$


2. Regra de Leibniz graduada: se $A$ é um a-multivetor, $B$ é um b-multivetor e $C$ é um cmultivetor então

$$
\begin{aligned}
& {[A, B \wedge C]=[A, B] \wedge C+(-1)^{(a-1) b} B \wedge[A, C],} \\
& {[A \wedge B, C]=A \wedge[B, C]+(-1)^{(c-1) b}[A, C] \wedge B .}
\end{aligned}
$$

3. Identidade de Jacobi graduada: se $A$ é um a-multivetor, $B$ é um b-multivetor e $C$ é um c-multivetor então

$$
(-1)^{(a-1)(c-1)}[A,[B, C]]+(-1)^{(b-1)(a-1)}[B,[C, A]]+(-1)^{(c-1)(b-1)}[C,[A, B]]=0 .
$$

4. Se A é um campo de vetores e $B$ é um b-multivetor então

$$
[A, B]=\mathcal{L}_{A} B
$$

onde $\mathcal{L}_{A}$ é a derivada de Lie por A. Em particular temos que se $B$ é um campo de vetores então o colchete que definimos coincide com o colchete de Lie de campos. Se $B=f$ é uma função então $[A, f]=A(f)=d f(A)$.

Demonstração. A demonstração de 1 segue diretamente da definição.

2: A regra de derivação implica que

$$
\frac{\partial B \wedge C}{\partial \zeta_{i}}=B \frac{\partial C}{\partial \zeta_{i}}+(-1)^{c} \frac{\partial B}{\partial \zeta_{i}} C
$$

Logo

$$
\begin{aligned}
{[A, B \wedge C]=} & \sum_{i} \frac{\partial A}{\partial \zeta_{i}} \frac{\partial(B \wedge C)}{\partial x^{i}}-(-1)^{(a-1)(b+c-1)} \sum_{i} \frac{\partial(B \wedge C)}{\partial \zeta_{i}} \frac{\partial A}{\partial x^{i}} \\
= & \sum_{i} \frac{\partial A}{\partial \zeta_{i}} \frac{\partial B}{\partial x^{i}} C+\sum_{i} \frac{\partial A}{\partial \zeta_{i}} B \frac{\partial C}{\partial x^{i}}-(-1)^{(a-1)(b+c-1)} \sum_{i} B \frac{\partial C}{\partial \zeta_{i}} \frac{\partial A}{\partial x^{i}} \\
& -(-1)^{(a-1)(b+c-1)+c} \sum_{i} \frac{\partial B}{\partial \zeta_{i}} C \frac{\partial A}{\partial x^{i}} \\
= & \sum_{i} \frac{\partial A}{\partial \zeta_{i}} \frac{\partial B}{\partial x^{i}} C-(-1)^{(a-1)(b+c-1)+c+a c} \sum_{i} \frac{\partial B}{\partial \zeta_{i}} \frac{\partial A}{\partial x^{i}} C \\
& +(-1)^{(a-1) b}\left(-(-1)^{(a-1)(c-1)} \sum_{i} B \frac{\partial C}{\partial \zeta_{i}} \frac{\partial A}{\partial x^{i}}+\sum_{i} B \frac{\partial A}{\partial \zeta_{i}} \frac{\partial C}{\partial x^{i}}\right) \\
= & {[A, B] \wedge C+(-1)^{(a-1) b} B \wedge[A, C] }
\end{aligned}
$$

e

$$
\begin{aligned}
{[A \wedge B, C]=} & -(-1)^{(c-1)(a+b-1)}[C, A \wedge B] \\
= & -(-1)^{(c-1)(a+b-1)}[C, A] \wedge B-(-1)^{(c-1)(a+b-1)}(-1)^{(c-1) a} A \wedge[C, B] \\
= & -(-1)^{(c-1)(a+b-1)}\left(-(-1)^{(a-1)(c-1)}\right)[A, C] \wedge B \\
& -(-1)^{(c-1)(a+b-1)}(-1)^{(c-1) a}\left(-(-1)^{(b-1)(c-1)}\right) A \wedge[B, C] \\
= & A \wedge[B, C]+(-1)^{(c-1) b}[A, C] \wedge B .
\end{aligned}
$$

3: Calculando diretamente temos

$$
(-1)^{(a-1)(c-1)}[A,[B, C]]=S_{1}+S_{2}+S_{3}+S_{4}
$$


onde

$$
\begin{aligned}
S_{1} & =(-1)^{(a-1)(c-1)} \sum_{i, j} \frac{\partial A}{\partial \zeta_{j}} \frac{\partial^{2} B}{\partial x_{j} \partial \zeta_{i}} \frac{\partial C}{\partial x_{i}}-(-1)^{(a-1)(b-1)} \sum_{i, j} \frac{\partial B}{\partial \zeta_{i}} \frac{\partial^{2} C}{\partial x_{i} \partial \zeta_{j}} \frac{\partial A}{\partial x_{j}}, \\
S_{2} & =(-1)^{(a-1)(c-1)} \sum_{i, j} \frac{\partial A}{\partial \zeta_{j}} \frac{\partial B}{\partial \zeta_{i}} \frac{\partial^{2} C}{\partial x_{i} x_{j}}-(-1)^{(c-1)(b-1)} \sum_{i, j} \frac{\partial C}{\partial \zeta_{i}} \frac{\partial A}{\partial \zeta_{j}} \frac{\partial^{2} B}{\partial x_{i} \partial x_{j}}, \\
S_{3} & =(-1)^{(b-1)(a-1)} \sum_{i, j} \frac{\partial^{2} B}{\partial \zeta_{j} \partial x_{i}} \frac{\partial C}{\partial \zeta_{i}} \frac{\partial A}{\partial x_{j}}-(-1)^{(c-1)(b-1)} \sum_{i, j} \frac{\partial^{2} C}{\partial \zeta_{i} \partial x_{j}} \frac{\partial A}{\partial \zeta_{j}} \frac{\partial B}{\partial x_{i}}, \\
S_{4} & =(-1)^{(b-1)(a+c)+b} \sum_{i, j} \frac{\partial^{2} C}{\partial \zeta_{i} \partial \zeta_{j}} \frac{\partial A}{\partial x_{i}} \frac{\partial B}{\partial x_{j}}-(-1)^{(a-1)(b-1)+c} \sum_{i, j} \frac{\partial^{2} B}{\partial \zeta_{j} \partial \zeta_{i}} \frac{\partial C}{\partial x_{i}} \frac{\partial A}{\partial x_{j}} \\
& =(-1)^{(b-1)(c-1)+a} \sum_{i, j} \frac{\partial^{2} C}{\partial \zeta_{j} \partial \zeta_{i}} \frac{\partial A}{\partial x_{i}} \frac{\partial B}{\partial x_{j}}-(-1)^{(a-1)(b-1)+c} \sum_{i, j} \frac{\partial^{2} B}{\partial \zeta_{j} \partial \zeta_{i}} \frac{\partial C}{\partial x_{i}} \frac{\partial A}{\partial x_{j}} .
\end{aligned}
$$

Cada um dos $S_{i}$ será zero quando somados com um termo similar de $(-1)^{(b-1)(a-1)}[B,[C, A]]$ e $(-1)^{(c-1)(b-1)}[C,[A, B]]$.

4: Se $f$ é uma função e $X=\sum_{i} \xi_{i} \frac{\partial}{\partial x_{i}}$ é um campo vetorial, então $\frac{\partial f}{\partial \zeta_{i}}=0$ e $[X, f]=$ $\sum \frac{\partial X}{\partial \zeta_{i}} \frac{\partial f}{\partial x_{i}}=\sum \xi \frac{\partial f}{\partial x_{i}}=X(f)$. Claramente da definição do colchete segue que ele coincide com o colchete de Lie quando $a=b=1$. Se $A$ é um campo vetorial e $B$ é um $b$-multivetor, $[A, B]=\mathcal{L}_{A} B$ segue por indução usando a regra de Leibniz.

Observe que o colchete definido anteriormente depende de uma escolha de coordenadas locais a princípio. Porém da regra de Leibniz segue que o cálculo do colchete de multivetores se reduz ao cálculo de colchetes de Lie de campos de vetores. Como o colchete de Lie de campos de vetores não depende da escolha de coordenadas locais segue que o colchete de multivetores não depende da escolha de coordenadas locais. Logo dados multivetores $A, B$ tem-se que $[A, B]$ é um multivetor bem definido.

Definição 1.3.1. Se $M$ é uma variedade suave, $A$ é um $a$-multivetor e $B$ é um $b$-multivetor, então o único $(a+b-1)$-multivetor $[A, B]$ definido anteriormente em cada sistema de coordenadas locais é chamado o colchete de Schouten de $A$ e $B$.

O colchete de Schouten nos dá uma forma muito conveniente de caracterizar estruturas de Poisson e campos Hamiltonianos.

Teorema 1.3.2. Um bivetor $\Pi$ é um bivetor de Poisson se e somente se

$$
[\Pi, \Pi]=0 \in \Gamma\left(\Lambda^{3} T M\right) .
$$

Se П é um bivetor de Poisson e $f \in C^{\infty}(M)$ é uma função, o campo Hamiltoniano associado a $f$ satisfaz

$$
X_{f}=-[\Pi, f] .
$$

Demonstração. Se em um sistema de coordenadas locais $\left(x_{1}, \ldots, x_{n}\right)$ temos

$$
\Pi=\sum_{i<j} \Pi_{i j} \frac{\partial}{\partial x_{i}} \wedge \frac{\partial}{\partial x_{j}}
$$

então

$$
-[\Pi, f]=-\left[\Pi=\sum_{i<j} \Pi_{i j} \frac{\partial}{\partial x_{i}} \wedge \frac{\partial}{\partial x_{j}}, f\right]=-\sum_{i j} \Pi_{i j} \frac{\partial}{\partial x_{i}} \frac{\partial f}{\partial x_{j}}=\sum_{i j} \Pi_{i j} \frac{\partial f}{\partial x_{i}} \frac{\partial}{\partial x_{j}}=X_{f} .
$$




\subsection{Ações de Grupo e Redução}

Lembre que uma ação à esquerda de um grupo de Lie $G$ em uma variedade suave $M$ é uma aplicação suave $\rho: G \times M \rightarrow M$ tal que

$$
\rho(e, p)=p, \forall p \in M
$$

onde $e$ é a identidade do grupo $G$ e

$$
\rho(g h, p)=\rho(g, \rho(h, p)), \forall g, h \in G, \forall p \in M .
$$

De forma análoga uma ação à direita de um grupo de Lie $G$ em uma variedade suave $M$ é uma aplicação suave $\rho: M \times G \rightarrow M$ tal que

$$
\rho(p, e)=p, \forall p \in M
$$

e

$$
\rho(p, g h)=\rho(\rho(p, g), h), \forall g, h \in G, \forall p \in M .
$$

Motivados pelo fato de os espaços de fase muitas vezes admitirem simetrias somos levados ao estudo de ações de grupos de Lie em variedades de Poisson. Se um grupo de Lie $G$ age em uma variedade de Poisson $(M, \pi)$ de forma que a ação preserva a estrutura de Poisson $\pi$ então o espaço $C^{\infty}(M)^{G}$ de funções $G$-invariantes em $M$ é uma álgebra de Poisson no seguinte sentido

Definição 1.4.1. Uma estrutura de Poisson em uma álgebra associativa comutativa $A$ é um colchete anti-simétrico $\{\cdot, \cdot\}: A \times A \rightarrow A$ que satisfaz a identidade de Leibniz e a identidade de Jacobi. Dizemos que uma álgebra com uma estrutura de Poisson é uma álgebra de Poisson.

Lembre que uma ação de um grupo de Lie $G$ em uma variedade suave $M$ é própria se a aplicação $\rho: G \times M \rightarrow M \times M$ é própria. Em particular, se $G$ é compacto a ação sempre é própria. Se a ação for livre e própria sabemos que o quociente $M / G$ é uma variedade e podemos identificar $C^{\infty}(M / G)$ com $C^{\infty}(M)^{G}$ através do pullback pela aplicação quociente $M \rightarrow M / G$. Portanto se $M$ é uma variedade de Poisson temos que $M / G$ herda uma estrutura de Poisson de forma que a aplicação $M \rightarrow M / G$ é um morfismo de Poisson, esta estrutura é chamada estrutura de Poisson reduzida. Observe que se tivermos um campo Hamiltoniano $X_{h}$ em $M$, com $h \in C^{\infty}(M)^{G}$, podemos projetar este campo para um campo Hamiltoniano $X_{\tilde{h}}$ em $M / G$ através da projeção $M \rightarrow M / G$. Note que $\operatorname{dim} M / G=\operatorname{dim} M-\operatorname{dim} G$ e portanto para encontrar as curvas integrais de $X_{\tilde{h}}$ precisaremos resolver um número menor de equações com menos variáveis do que para encontrar as curvas integrais de $X_{h}$, e portanto uma simplificação do sistema Hamiltoniano dado por $h$.

Exemplo 1.4.1. Suponha que $S^{1}$ age em $S^{2}$ por rotações em torno de um eixo fixado. Temos que essa ação é de Poisson pois ela preserva a estrutura simplética dada pela forma volume. Como esta ação não é livre (todos elementos do grupo fixam o ponto $(0,0)$ ) temos que o espaço quociente não é uma variedade suave. Neste caso, veremos no Capítulo 4 que não temos uma estrutura de Poisson no quociente porém teremos uma estrutura de Poisson não comutativa.

\subsection{Cohomologia de Poisson}

Veremos nesta seção que podemos definir uma cohomologia para variedades de Poisson utilizando o colchete de Schouten. No capítulo 4 veremos que também podemos definir uma cohomologia de Poisson para estruturas de Poisson não comutativas de forma análoga, utilizando o colchete de Gerstenhaber.

Baseados no seguinte lema podemos definir uma cohomologia de variedades de Poisson. 
Lema 1.5.1. Seja $(M, \pi)$ uma variedade de Poisson e A um a-campo multivetorial. Então

$$
[\pi,[\pi, A]]=0,
$$

onde $[\cdot, \cdot]$ é o colchete de Schouten.

Demonstração. Pela identidade de Jacobi graduada temos

$$
(-1)^{(a-1)}[\pi,[\pi, A]]-[\pi,[A, \pi]]+(-1)^{(a-1)}[A,[\pi, \pi]]=0 .
$$

Observe que $[A, \pi]=-(-1)^{(a-1)}[\pi, A]$ e portanto $[\pi,[\pi, A]]=-\frac{1}{2}[A,[\pi, \pi]]$. Como $\pi$ é um bivetor de Poisson temos $[\pi, \pi]=0$ e portanto $[\pi,[\pi, A]]=0$.

Seja $(M, \pi)$ uma variedade de Poisson. Seja

$$
\delta_{\pi}: \Lambda^{*}(T M) \rightarrow \Lambda^{*}(T M)
$$

a aplicação dada por

$$
\delta_{\pi}(A)=[\pi, A]
$$

Do lema anterior segue que

$$
\delta_{\pi} \circ \delta_{\pi}=0
$$

O complexo

$$
\cdots \stackrel{\delta_{\pi}}{\longrightarrow} \Lambda^{k-1}(T M) \stackrel{\delta_{\pi}}{\longrightarrow} \Lambda^{k}(T M) \stackrel{\delta_{\pi}}{\longrightarrow} \Lambda^{k+1}(T M) \stackrel{\delta_{\pi}}{\longrightarrow} \cdots
$$

é chamado complexo de Lichnerowicz e a cohomologia do complexo é chamada cohomologia de Poisson de $(M, \pi)$. Denotaremos os grupos de cohomologia de Poisson de $(M, \pi)$ por $H_{\pi}^{p}(M)$.

O grupo de cohomologia de Poisson de grau zero $H_{\pi}^{0}(M)$ é o grupo de funções $f \in C^{\infty}(M)$ tais que $X_{f}=-[\pi, f]=0$, tais funções são chamadas funções de Casimir, observe que estas funções são as primeiras integrais de campos Hamiltonianos.

O grupo de cohomologia de Poisson de grau $1 H_{\pi}^{1}(M)$ é o quociente do espaço dos campos de vetores de Poisson, isto é, os campos de vetores $X$ que satisfazem $[\pi, X]=0$, pelos campos de vetores Hamiltonianos, isto é, campos $X$ da forma $X=[\pi, f]$ para alguma $f \in C^{\infty}(M)$. Dizemos que um campo vetorial $X$ é um campo de Poisson se, e somente se, satisfaz $X\{f, g\}=$ $\{X f, g\}+\{f, X g\}$, isto é, se $X$ é uma derivação do colchete de Poisson ou se $[\pi, X]=0$. Note também que os campos Hamiltonianos são derivações internas do colchete.

O segundo grupo de cohomologia de Poisson $H_{\pi}^{2}(M)$ é o quociente do espaço dos bivetores $\Lambda$ que satisfazem $[\pi, \Lambda]=0$ pelo espaço dos bivetores $\Lambda$ da forma $\Lambda=[\pi, X]$, onde $X$ é um campo vetorial. Se $[\pi, \Lambda]=0$ e $\epsilon$ é uma variável formal, então $\pi+\epsilon \Lambda$ satisfaz

$$
[\pi+\epsilon \Lambda, \pi+\epsilon \Lambda]=\epsilon^{2}[\Lambda, \Lambda]=0 \bmod \epsilon^{2} .
$$

Portanto podemos interpretar $\pi+\epsilon \Lambda$ como uma deformação infinitesimal de $\pi$ no espaço dos bivetores de Poisson. Por outro lado, temos que

$$
\left(\varphi_{Y}^{\epsilon}\right)_{*} \pi=\pi+\epsilon \frac{d}{d \epsilon} \varphi_{Y}^{\epsilon}+\cdots=\pi+\epsilon\left(-\mathcal{L}_{Y} \pi\right) \bmod \epsilon^{2}=\pi+\epsilon[\pi, Y] \bmod \epsilon^{2}
$$

onde $\left(\varphi_{Y}^{\epsilon}\right)$ é o fluxo no tempo $\epsilon$ de um campo de vetores $Y$. Logo $\pi+\epsilon[\pi, Y]$ é uma deformação infinitesimal dada por um difeomorfismo e chamaremos essas deformações de triviais. Logo $H_{\pi}^{2}(M)$ é o espaço de todas as deformações de $\pi$ quocientado pelas deformações triviais. 
Definição 1.5.1. Uma deformação de Poisson de um bivetor de Poisson $\pi$ é uma série de potências formal

$$
\pi_{\epsilon}=\pi+\sum_{i=1}^{\infty} \epsilon^{i} \pi_{i}
$$

tal que $\left[\pi_{\epsilon}, \pi_{\epsilon}\right]=0$, onde $\pi_{i}$, para cada $i$, é um bivetor em $M$. Chamamos $\pi_{1}$ de infinitesimal associado à deformação.

Tomando $\pi_{0}=\pi$ temos

$$
\left[\pi_{\epsilon}, \pi_{\epsilon}\right]=\sum_{n=0}^{\infty} \epsilon^{n}\left(\sum_{i+j=n}\left[\pi_{i}, \pi_{j}\right]\right)
$$

Logo

$$
\begin{aligned}
{\left[\pi_{\epsilon}, \pi_{\epsilon}\right]=0 } & \Longleftrightarrow \sum_{i+j=n}\left[\pi_{i}, \pi_{j}\right]=0, \text { para todo } n \\
& \Longleftrightarrow 2 \delta_{\pi} \pi_{n}=-\sum_{i+j=n, i \geq 1, j \geq 1}\left[\pi_{i}, \pi_{j}\right], \text { para todo } n
\end{aligned}
$$

Desta equação segue que $\delta_{\pi}\left(\pi_{1}\right)=0$ e temos a seguinte proposição que nos permite interpretar $H_{\pi}^{3}(M)$ como obstruções para existência de deformações de $\pi$

Proposição 1.5.2. Dado $\pi_{1}$ bivetor em $M$ tal que $\delta_{\pi}\left(\pi_{1}\right)=0$ a obstrução para existência de uma deformação de Poisson com infinitesimal $\pi_{1}$ é $H_{\pi}^{3}(M)$. Isto é, se $H_{\pi}^{3}(M)=0$ então existe uma deformação de Poisson com infinitesimal $\pi_{1}$.

Demonstração. Vamos mostrar a existência de uma sequência $\left\{\pi_{n}\right\}_{n=2}^{\infty}$ que satisfaz a equação $\llbracket 2$ por indução. Suponha que encontramos $\pi_{2}, \ldots, \pi_{k-1}$ que satifazem ए2. Seja

$$
E_{k}=-\sum_{i+j=k, i \geq 1, j \geq 1}\left[\pi_{i}, \pi_{j}\right]
$$

Para mostrar que existe um $\pi_{k}$ que satisfaz a equação $\amalg .2$ basta mostrar que $\delta_{\pi}\left(E_{k}\right)=0$ pois $H_{\pi}^{3}(M)=0$. Temos

$$
\begin{aligned}
\delta_{\pi}\left(E_{k}\right) & =\sum_{i+j=k, i \geq 1, j \geq 1}\left(\left[\pi_{i}, \delta_{\pi} \pi_{j}\right]+\left[\pi_{j}, \delta_{\pi} \pi_{i}\right]\right) \\
& =2 \sum_{i+j=k, i \geq 1, j \geq 1}\left[\pi_{j}, \delta_{\pi} \pi_{i}\right] \\
& =-\sum_{i+j=k, i \geq 1, j \geq 1}\left(\sum_{\alpha+\beta=i, \alpha \geq 1, \beta \geq 1}\left[\pi_{j},\left[\pi_{\alpha}, \pi_{\beta}\right]\right]\right) \\
& =-\sum_{\alpha+\beta+j=k, \alpha \geq 1, \beta \geq 1, j \geq 1}\left[\pi_{j},\left[\pi_{\alpha}, \pi_{\beta}\right]\right] \\
& =-\frac{1}{3} \sum_{\alpha+\beta+j=k, \alpha \geq 1, \beta \geq 1, j \geq 1}\left(\left[\pi_{j},\left[\pi_{\alpha}, \pi_{\beta}\right]\right]+\left[\pi_{\alpha},\left[\pi_{\beta}, \pi_{j}\right]\right]+\left[\pi_{\beta},\left[\pi_{j}, \pi_{\alpha}\right]\right]\right) \\
& =0 .
\end{aligned}
$$




\subsection{Cohomologia de Poisson e Cohomologia de de Rham}

Note que uma estrutura de Poisson $\pi$ em uma variedade $M$ nos dá um homomorfismo de fibrados vetoriais

$$
\sharp: T^{*} M \rightarrow T M
$$

que associa a cada forma $\alpha$ o vetor $\sharp(\alpha)$ que satisfaz a seguinte identidade para cada forma $\beta$

$$
\pi(\alpha, \beta)=\beta(\sharp(\alpha)) .
$$

Este homomorfismo é um isomorfismo se, e somente se, $\pi$ é não degenerada, isto é, se $\pi$ é uma estrutura simplética em $M$.

Podemos estender este homomorfismo a um homomorfismo

$$
\sharp: \Lambda^{k}\left(T^{*} M\right) \rightarrow \Lambda^{k}(T M)
$$

tomando potências exteriores e a uma aplicação $C^{\infty}(M)$-linear

$$
\sharp: \Omega^{k}(M) \rightarrow \mathcal{V}^{k}(M)
$$

de formas diferenciais suaves em $M$ para campos de multivetores suaves em $M$. Se $k=0 \sharp$ é a identidade.

Temos o seguinte lema que relaciona o complexo de de Rham com o complexo de Lichnerowicz

Lema 1.6.1. Dada uma forma diferencial $\omega$ em uma variedade de Poisson $(M, \pi)$ vale

$$
\sharp(d \omega)=-[\pi, \sharp(\omega)]=-\delta_{\pi}(\sharp(\omega)) .
$$

Demonstração. Provaremos este lema por indução no grau de $\omega$. Se $\omega$ é uma função temos $\sharp(\omega)=\omega$ e $\sharp(\mathrm{d} \omega)=X_{\omega}$, onde $X_{\omega}$ é o campo Hamiltoniano associado a $\omega$, logo $\sharp(\mathrm{d} \omega)=-[\pi, \omega]=$ $-[\pi, \sharp(\omega)]$ e portanto a equação $\llbracket .3$ é satisfeita. Se $\omega=\mathrm{d} f$ é uma 1-forma exata temos $\sharp(\mathrm{d} \omega)=0$ e $[\pi, \sharp(\omega)]=\left[\pi, X_{f}\right]=0$ e portanto satisfaz a equação [1.3.

Agora se a equação [.3] é satisfeita por uma $p$-forma $\eta$ e uma $q$-forma $\omega$ então $\eta \wedge \omega$ também satisfaz a equação. De fato, temos

$$
\begin{aligned}
\sharp(d(\eta \wedge \omega)) & =\sharp\left(\mathrm{d} \eta \wedge \omega+(-1)^{p} \eta \wedge \mathrm{d} \omega\right)=\sharp(\mathrm{d} \eta) \wedge \sharp(\omega)+(-1)^{p} \sharp(\eta) \wedge \sharp(\mathrm{d} \omega) \\
& =-[\pi, \sharp(\eta)] \wedge \sharp(\omega)-(-1)^{p} \sharp(\eta) \wedge[\pi, \sharp(\omega)]=-[\pi, \sharp(\eta) \wedge \sharp(\omega)] \\
& =-[\pi, \sharp(\eta \wedge \omega)] .
\end{aligned}
$$

O lema acima nos mostra que exceto por um sinal a aplicação $\sharp$ leva o operador diferencial d do complexo de de Rham no operador $\delta_{\pi}$ do complexo de Lichnerowicz. Em particular $\sharp$ induz um homomorfismo em cohomologia. Portanto temos

Teorema 1.6.2. Seja $(M, \pi)$ uma variedade de Poisson. Existe um homomorfismo natural

$$
\sharp^{*}: H_{d R}^{*}(M) \rightarrow H_{\pi}^{*}(M)
$$

da cohomologia de de Rham para a cohomologia de Poisson induzido pela aplicação \#. Se M for uma variedade simplética $\sharp^{*}$ é um isomorfismo.

Demonstração. Note que quando $M$ é simplética, $\sharp$ é um isomorfismo e portanto $\sharp^{*}$ também é. 


\section{Capítulo 2}

\section{Geometria Não Comutativa}

Do ponto de vista de conjuntos, para formar um espaço quociente $X / \sim$ precisamos apenas de uma relação de equivalência $\sim$ em um conjunto $X$. Se $X$ for um espaço topológico temos uma topologia canônicamente associada ao espaço quociente. Se $X$ possuir mais estrutura, por exemplo se $X$ for uma variedade suave, queremos que o espaço quociente também possua a mesma estrutura. Quando isso ocorre dizemos que temos um bom quociente, caso contrário temos um quociente ruim. O quociente de uma variedade suave facilmente pode não ser uma variedade suave, em particular quando este quociente é por uma ação de grupo. Então o problema que a geometria não comutativa procura atacar é como lidar com estes quocientes ruins.

A solução do problema, proposta por Connes (Connes, 1994), é aumentar a categoria de espaços clássicos para espaços não comutativos. Esta proposta é baseada no fato de que uma relação de equivalência é normalmente obtida de uma estrutura mais complexa esquecendo parte desta estrutura. Por exemplo, uma relação de equivalência $\sim$ vem de uma ação de um grupo $G$ em um espaço $X$ onde $x \sim y$ se, e somente se, $g x=y$ para algum $g \in G$. Note que podem existir muitos $g$ que satisfazem $g x=y$, em particular podem existir muitos $g$ que satisfazem $g x=x$. Quando formamos a relação de equivalência esta informação é perdida. A ideia para organizar toda esta informação é guardá-la em um grupoide, que pode ser topologico, suave, discreto. Desta forma o quociente não comutativo será dado pela álgebra do grupoide associado.

Dependendo da aplicação que temos em mente podemos utilizar a álgebra de grupoide puramente algébrica, suave, contínua, mensurável, $C^{*}$-algébrica, etc. A escolha será ditada pelo problema que temos em mente. No nosso caso estaremos interessados na álgebra de grupoide suave.

O motivo desta abordagem ser razoável é o fato de que quando temos um bom quociente, a álgebra de funções no quociente é Morita equivalente à álgebra de grupoide. Como sabemos que álgebras Morita equivalentes possuem cohomologia de Hochschild, cohomologia cíclica, $K$-teoria isomorfas temos que invariantes topológicos definidos usando geometria não comutativa são os mesmos para quocientes classicos e não comutativos.

\section{$2.1 \quad$ Grupoides}

Definição 2.1.1. Um grupoide é uma categoria pequena em que todos os morfismos são isomorfismos.

Lembre que dizemos que uma categoria é pequena quando seus objetos e morfismos formam um conjunto e não uma classe.

Seja $\mathscr{G}$ um grupoide, denotaremos o conjunto de objetos de $\mathscr{G}$ por $\mathscr{G}^{(0)}$ e também usaremos o símbolo $\mathscr{G}$ para denotar o conjunto de morfismos de $\mathscr{G}$.

Temos as funções

$$
s: \mathscr{G} \rightarrow \mathscr{G}^{(0)}, t: \mathscr{G} \rightarrow \mathscr{G}^{(0)} \text { e } i: \mathscr{G} \rightarrow \mathscr{G}
$$


que levam cada morfismo em sua origem, seu destino e seu inverso, respectivamente.

Também temos a função

$$
u: \mathscr{G}^{(0)} \rightarrow \mathscr{G}
$$

que leva cada objeto ao morfismo unitário deste objeto em si mesmo.

Note que a composição de morfismos só está definida no conjunto

$$
\mathscr{G}^{(2)}=\left\{\left(\gamma_{1}, \gamma_{2}\right) ; s\left(\gamma_{1}\right)=t\left(\gamma_{2}\right)\right\} .
$$

Exemplo 2.1.1. Seja $\mathscr{G}$ a categoria com um único objeto $*$ e com

$$
\operatorname{Hom}_{\mathscr{G}}(*, *)=G
$$

onde $G$ é um grupo e a composição de morfismos é dada pela multiplicação do grupo. Então temos que $\mathscr{G}$ é um grupoide.

Exemplo 2.1.2. Seja

$$
G \times X \rightarrow X, \quad(g, x) \mapsto g x
$$

a ação de um grupo $G$ em um conjunto $X$.

Defina o grupoide

$$
\mathscr{G}=X \rtimes G
$$

chamado grupoide de ação tomando

$$
\mathscr{G}^{(0)}=X \quad e \quad \operatorname{Hom}_{\mathscr{G}}(x, y)=\{g \in G ; g x=y\}
$$

e a composição de morfismos como a multiplicação do grupo.

Observe que podemos identificar o conjunto de morfismos de $\mathscr{G} \operatorname{com} X \times G$ com a composição de morfismos dada por

$$
(g x, h) \circ(x, g)=(x, h g) .
$$

Note que o exemplo [.].] corresponde à ação do grupo $G$ em um ponto.

Exemplo 2.1.3. Seja $X$ um conjunto e $\sim$ uma relação de equivalência em $X$. Definimos o grupoide $\mathscr{G}$ chamado gráfico de $\sim$ da seguinte maneira. Seja

$$
\mathscr{G}^{(0)}=X,
$$

e seja

$$
\operatorname{Hom}_{\mathscr{G}}(x, y)= \begin{cases}(x, y) & \text { se } x \sim y \\ \emptyset & \text { caso contrário }\end{cases}
$$

Note que o conjunto de morfismo se identifica com o gráfico da relação :

$$
\mathscr{G}=\{(x, y) ; x \sim y\} \subset X \times X
$$

Exemplo 2.1.4. Um caso particular do exemplo anterior é dado pelo grupoide dos pares. Seja $X$ um conjunto finito, definiremos o grupoide dos pares $\mathscr{G}$ da seguinte maneira: temos $\mathscr{G}^{(0)}=X$ e $\mathscr{G}=X \times X$, e as aplicações $s, t$ são dadas pelas projeções.

Para falar de exemplos interessantes como grupoides associados a ações contínuas de grupos e a folheações precisaremos falar de grupoides topológicos e suaves. 
Definição 2.1.2. Um grupoide $\mathscr{G}$ é um grupoide topológico se $\mathscr{G}^{(0)}$ e o conjunto de morfismos $\mathscr{G}$ são espaços topológicos e a composição, a função origem, a função destino e a função inversão são contínuas.

Dizemos que um grupoide topológico $\mathscr{G}$ é um grupoide étale se $\mathscr{G}^{(0)}$ é um espaço Hausdorff localmente compacto e a função origem $s$ é uma função étale, i.e. é um homeomorfismo local. Observe que se $s$ for étale então $t$ também é.

Um grupoide de Lie é um grupoide $\mathscr{G}$ tal que $\mathscr{G}^{(0)}$ e o conjunto de morfismos $\mathscr{G}$ são variedades suaves, as funções $u, s, t, i$ e a composição $\mathscr{G}^{(2)} \rightarrow \mathscr{G}$ são suaves e $s$ e $t$ são submersões. Note que a última condição garante que $\mathscr{G}^{(2)}$ é uma variedade suave.

Exemplo 2.1.5. Seja $G$ um grupo discreto agindo em um espaço Hausdorff localmente compacto $X$. Seja $\mathscr{G}=X \rtimes G$ o grupoide de transformação. Podemos colocar a topologia produto em $\mathscr{G}=X \times G$. Temos facilmente que $s, t$ e a composição $\circ$ são contínuas e as $s$-fibras são subconjuntos discretos de $X \times G$, portanto $s$ é étale e $\mathscr{G}$ é um grupoide étale.

Exemplo 2.1.6. Seja $X$ um espaço topológico. Definimos o grupoide $\Pi_{1}(X)$, chamado grupoide fundamental de $X$, da seguinte maneira. O conjunto de objetos de $\Pi_{1}(X)$ é $X$ e, para todo $x, y \in X$, os morfismos de $x$ para $y$ são as classes de homotopia de caminhos contínuos de $x$ para $y$. Isto define um grupoide com a composição sendo o concatenamento de caminhos. Usando a topologia compacto-aberto no conjunto das aplicações $[0,1] \rightarrow X$ podemos colocar uma topologia em $\Pi_{1}(X)$ tomando o quociente da topologia compacto-aberto pela relação de equivalência definida pela homotopia.

\section{2 Álgebras de grupoides}

À uma relação de equivalência em um espaço podemos associar um grupoide e a idéia da geometria não comutativa é tomar a álgebra de grupoide deste grupoide associado como a álgebra de funções no espaço quociente.

Nesta seção veremos o que é a álgebra de grupoide de um grupoide e calcular alguns exemplos.

Seja $\mathscr{G}$ um grupoide discreto e seja

$$
\mathbb{C} \mathscr{G}=\bigoplus_{\gamma \in \mathscr{G}} \mathbb{C} \gamma
$$

o espaço vetorial gerado por $\mathscr{G}$. Portanto um elemento de $\mathbb{C} \mathscr{G}$ é uma soma $\sum a_{\gamma} \gamma$ onde $\gamma \in \mathscr{G}$ e $a_{\gamma}=0$ para quase todo $\gamma$.

A fórmula

$$
\gamma_{1} \gamma_{2}= \begin{cases}\gamma_{1} \circ \gamma_{2} & \text { se } \gamma_{1} \circ \gamma_{2} \text { está definido, } \\ 0 & \text { caso contrário }\end{cases}
$$

define uma estrutura de álgebra associativa em $\mathbb{C} \mathscr{G}$.

Exemplo 2.2.1. Se o grupoide $\mathscr{G}$ possui apenas um objeto, com grupo de automorfismos $G$, temos que a álgebra de grupoide é apenas a álgebra de grupo

$$
\mathbb{C} \mathscr{G}=\mathbb{C} G \text {. }
$$

Exemplo 2.2.2. Seja $X$ um conjunto finito e $\mathscr{G}$ o grupoide dos pares sobre $X$. Neste caso temos

$$
\mathbb{C} \mathscr{G} \cong \text { End } V
$$

onde $V$ é o espaço vetorial gerado por $X$. Para ver isso, assuma que $X=\{1,2, \ldots, n\}$ é um conjunto de $n$ elementos. Podemos escrever os morfismos de $\mathscr{G}$ da seguinte forma

$$
\mathscr{G}=\{(i, j) ; i, j=1, \ldots, n\},
$$


com a composição dada por

$$
(l, k) \circ(j, i)=(l, i), \quad \text { se } j=k .
$$

A aplicação

$$
\sum a_{i, j}(i, j) \mapsto \sum a_{i, j} E_{i, j}
$$

onde $E_{i, j}$ é a matriz com 1 na entrada $(i, j)$ e 0 nas outras, define um isomorfismo de álgebras entre $\mathbb{C} \mathscr{G}$ e a álgebra de matrizes $M_{n}(\mathbb{C})$.

Exemplo 2.2.3. Neste exemplo veremos que a álgebra de grupoide de qualquer grupoide discreto é dada em termos dos dois exemplos anteriores.

Dizemos que um grupoide $\mathscr{G}$ é transitivo se para quaisquer dois objetos $x, y$ de $\mathscr{G}$ existe um morfismo de $x$ para $y$. Podemos ver facilmente que cada grupoide pode ser decomposto como uma união de grupoides transitivos

$$
\mathscr{G}=\bigcup_{i} \mathscr{G}_{i}
$$

Com essa decomposição obtemos

$$
\mathbb{C} \mathscr{G} \cong \bigoplus_{i} \mathbb{C}^{\mathscr{G}}
$$

Agora, seja $\mathscr{T}$ um grupoide transitivo e seja $x_{0}$ um objeto de $\mathscr{T}$. Tome

$$
G=\operatorname{Hom}_{\mathscr{T}}\left(x_{0}, x_{0}\right),
$$

o grupo de isotropia de $x_{0}$. Note que se $x_{1}$ é outro objeto de $\mathscr{T}$ e $f \in \operatorname{Hom}_{\mathscr{T}}\left(x_{0}, x_{1}\right)$ temos que a aplicação $\varphi \mapsto f \circ \varphi \circ f^{-1}$ é um isomorfismo entre $G$ e $\operatorname{Hom}_{\mathscr{T}}\left(x_{1}, x_{1}\right)$. Logo a classe de isomorfismo de $G$ independe do objeto $x_{0}$ escolhido.

Temos então um isomorfismo de grupoides

$$
\mathscr{T} \cong \mathscr{T}_{1} \times \mathscr{T}_{2}
$$

onde $\mathscr{T}_{1}$ é um grupoide com um objeto com grupo de automorfismo $G$ e $\mathscr{T}_{2}$ é o grupoide dos pares sobre o conjunto de objetos de $\mathscr{T}$. Assumindo que o conjunto de objetos de $\mathscr{T}$ é finito temos

$$
\mathbb{C} \mathscr{T} \cong \mathbb{C} \mathscr{T}_{1} \otimes \mathbb{C} \mathscr{T}_{2} \cong \mathbb{C} G \otimes M_{n}(\mathbb{C}),
$$

onde $n$ é o número de elementos de $T$.

Logo segue que dado um grupoide discreto $\mathscr{G}$ temos, quando cada componente transitiva de $\mathscr{G}$ é finita,

$$
\mathbb{C} \mathscr{G} \cong \bigoplus_{i} \mathbb{C} G_{i} \otimes M_{n_{i}}(\mathbb{C})
$$

onde a soma é tomada sobre as componentes transitivas de $\mathscr{G}, n_{i}$ é a cardinalidade da componente e $G_{i}$ é o grupo de isotropia correspondente.

A seguir daremos outra forma de descrever a álgebra de grupoide que é mais propícia à generalização ao caso topológico. Temos um isomorfismo

$$
\mathbb{C} \mathscr{G} \cong\{f: \mathscr{G} \rightarrow \mathbb{C} ; f \text { tem suporte finito }\}
$$

e sob este isomorfismo o produto será o produto de convolução

$$
(f * g)(\gamma)=\sum_{\gamma_{1} \gamma_{2}=\gamma} f\left(\gamma_{1}\right) g\left(\gamma_{2}\right)=\sum_{\gamma_{1} \in t^{-1}(\gamma)} f\left(\gamma_{1}\right) g\left(\gamma_{1}^{-1} \gamma\right)
$$

Com esta definição podemos, dependendo da estrutura do grupoide, tomar a álgebra de grupoide correspondente. Por exemplo, se $\mathscr{G}$ for um grupoide topológico podemos tomar a álgebra 
de grupoide $C_{c}(\mathbb{G})=\{f: \mathscr{G} \rightarrow \mathbb{C} ; f$ é contínua e tem suporte compacto $\}$. Onde o produto será dado por

$$
(f * g)(\gamma)=\int_{\gamma_{1} \gamma_{2}=\gamma} f\left(\gamma_{1}\right) g\left(\gamma_{2}\right):=\int_{\gamma_{1} \in t^{-1}(\gamma)} f\left(\gamma_{1}\right) g\left(\gamma_{1}^{-1} \gamma\right) d \mu^{t(\gamma)},
$$

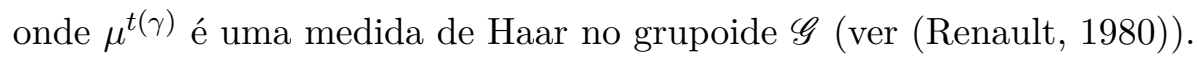

Quando $\mathscr{G}$ é o grupoide de ação associado à uma ação de grupo temos que a álgebra de grupoide é dada por uma álgebra de produto cruzado(ver (Khalkhali, 20109)). A seguir vamos ver como definimos esta álgebra. Primeiro vamos definir o que é o produto cruzado no caso puramente algébrico.

Seja $\operatorname{Aut}(A)$ o grupo de automorfismos da álgebra $A$. Uma ação de um grupo $G$ em $A$ é um homomorfismo de grupo

$$
\alpha: G \rightarrow \operatorname{Aut}(A)
$$

Usaremos a notação $g(a):=\alpha(g)(a)$.

A álgebra de produto cruzado $A \rtimes_{\alpha} G$ é definida da seguinte maneira. Como $\mathbb{C}$-espaço vetorial $A \rtimes_{\alpha} G$ é o espaço

$$
A \otimes \mathbb{C} G
$$

O produto é definido pela regra

$$
(a \otimes g)(b \otimes h)=a g(b) \otimes g h,
$$

para $a, b \in A$ e $g, h \in G$. Note que a associatividade desta álgebra segue diretamente da associatividade de $A$ e que $A \rtimes_{\alpha} G$ é unital se e somente se $A$ é unital e $G$ age preservando a unidade.

Equivalentemente podemos definir $A \rtimes_{\alpha} G$ como a álgebra universal gerada pelas álgebras $A$ e $\mathbb{C} G$ quocientada pela relação

$$
g a g^{-1}=g(a),
$$

para $a \in A$ e $g \in G$. De fato, observe que

$$
a g b h=a g b g^{-1} g h=a g(b) g h,
$$

para $a, b \in A$ e $g, h \in G$.

Note que dada uma representação $A \rtimes_{\alpha} G \rightarrow \operatorname{End}(V)$, onde $V$ é um $\mathbb{C}$-espaço vetorial, obtemos um par de representações $\pi: A \rightarrow \operatorname{End}(V)$ e $\rho: G \rightarrow \mathrm{GL}(V)$ que satisfazem

$$
\rho(g) \pi(a) \rho(g)^{-1}=\pi(g(a))
$$

onde $a \in A$ e $g \in G$.

Inversamente temos que dado um par de representações $\pi: A \rightarrow \operatorname{End}(V)$ e $\rho: G \rightarrow \operatorname{GL}(V)$ em um espaço vetorial $V$ que satisfazem a equação 2. obtemos uma representação $\rho \rtimes \pi$ de $A \rtimes_{\alpha} G$ em $V$ tomando

$$
(\rho \rtimes \pi)(a \otimes g)=\pi(a) \rho(g) .
$$

Desta forma obtemos uma correspondência entre representações de $A \rtimes_{\alpha} G$ e representações de $A$ e $G$ que satisfazem a equação 2.$]$.

Agora vamos descrever o análogo da construção acima para $C^{*}$-álgebras.

Definição 2.2.1. Um sistema covariante é uma tripla $(A, G, \alpha)$, onde $A$ é uma $C^{*}$-álgebra, $G$ é um grupo localmente compacto e $\alpha: G \rightarrow \operatorname{Aut}(A)$ é um homomorfismo contínuo, onde $\operatorname{Aut}(A)$ é o grupo de $*$-automorfismos de $A$ com a topologia da convergência pontual.

Note que a topologia em $\operatorname{Aut}(A)$ é tal que para cada $a \in A$ a aplicação

$$
G \ni g \mapsto g(a) \in A
$$

é contínua. 
Exemplo 2.2.4. Um exemplo de sistema covariante é obtido tomando $A=C_{0}(X)$ e a ação de $G$ induzida por uma ação em $X$ dada por $(g \cdot f)(x):=f\left(g^{-1} x\right)$.

Definição 2.2.2. Uma representação covariante de um sistema covariante $(A, G, \alpha)$ é uma tripla $(H, \pi, \rho)$, onde $(H, \pi)$ é uma $C^{*}$-representação de $A$ e $(H, \rho)$ é uma representação unitária de $G$ que satisfazem

$$
\rho(g) \pi(a) \rho(g)^{-1}=\pi(g(a))
$$

para todo $a \in A$ e $g \in G$.

A ideia para definir o produto cruzado $C^{*}$ é construir uma $C^{*}$-álgebra cujas representações estejam em correspondência com as representações covariantes de $(A, G, \alpha)$, de forma análoga ao caso puramente algébrico. Este problema possui uma solução que vamos indicar a seguir.

Seja $\mu$ uma medida de Haar à esquerda em $G$, vamos definir um produto e uma involução no espaço $C_{c}(G, A)$ de funções de $G$ para $A$ contínuas com suporte compacto. Dados $f, g \in C_{c}(G, A)$ e $t \in G$ defina

$$
(f \times g)(t)=\int_{G} f(s) \alpha(s) g\left(s^{-1} t\right) \mathrm{d} \mu(s)
$$

$\mathrm{e}$

$$
f^{*}(t)=\Delta(t)^{-1} \alpha(t)\left(f\left(t^{-1}\right)^{*}\right)
$$

Computando diretamente temos que essas definições nos dão um produto associativo e uma involução em $C_{c}(G, A)$.

Com a norma $\|f\|_{1}=\int_{G}\|f(t)\| \mathrm{d} \mu(t), C_{c}(G, A)$ é uma álgebra normada involutiva, denotaremos por $L^{1}(G, A)$ seu completamento que é uma álgebra de Banach, porém não é uma $C^{*}$-álgebra. Dada uma representação covariante $(H, \pi, \rho)$ de $(A, G, \alpha)$ podemos definir uma representação $\pi \rtimes \rho$ de $L^{1}(G, A)$ por

$$
(\pi \rtimes \rho)(f)=\int_{G} \pi(f(t)) \rho(t) \mathrm{d} \mu(t) .
$$

Definimos então a norma

$$
\|f\|=\sup \|(\pi \rtimes \rho)(f)\|,
$$

onde o supremo é tomado sobre todas representações covariantes $(H, \pi, \rho)$ de $(A, G, \alpha)$ e a norma à direita é a norma de operador. Esta norma é chamada de norma universal, o completamento de $C_{c}(G, A)$ com respeito à norma universal é chamado produto cruzado $C^{*}$ de $A$ por $G$ e é denotado por $A \rtimes_{\alpha} G$.

O seguinte teorema pode ser encontrado em (Williams, [2006)(Proposition 2.40).

Teorema 2.2.1. Seja $\alpha: G \rightarrow$ Aut $(A)$ uma ação de um grupo localmente compacto em uma $C^{*}$ álgebra $A$ e $H$ um espaço de Hilbert. Então temos uma bijeção entre representações de $A \rtimes_{\alpha} G$ em $H$ e representações covariantes de $(A, G, \alpha)$ em $H$. 


\section{Capítulo 3}

\section{Cohomologia de Hochschild}

\subsection{Definição de Homologia e Cohomologia de Hochschild}

Seja $A$ uma álgebra com 1 sobre um corpo $k$. Denotaremos por $A^{o p}$ a álgebra oposta de $A$, isto é, $A^{o p}=A$ como $k$-espaços vetoriais e $a \cdot{ }_{o p} b:=b a$ para todos $a, b$ em $A$. Caso não esteja indicado, os produtos tensoriais serão sobre $k$, isto é, $\otimes=\otimes_{k}$.

Seja $A^{e}=A \otimes A^{o p}$ com a multiplicação

$$
\left(a_{1} \otimes b_{1}\right) \cdot\left(a_{2} \otimes b_{2}\right)=a_{1} a_{2} \otimes b_{2} b_{1}
$$

para todos $a_{1}, a_{2}, b_{1}, b_{2}$ em $A$. Chamamos $A^{e}$ de álgebra envelopante de $A$.

Por $A$-bimódulo entendemos um espaço vetorial $M$ sobre $k$ que é ao mesmo tempo um $A$ módulo à esquerda e à direita de forma que vale

$$
\left(a_{1} m\right) a_{2}=a_{1}\left(m a_{2}\right)
$$

para todos $a_{1}, a_{2} \in A$ e $m \in M$, e as ações à esquerda e à direita de $k$ em $M$, induzidas pela aplicação $k \rightarrow A$ dada por $c \mapsto c \cdot 1$, coincidem.

Exemplo 3.1.1. A possui uma estrutura de $A$-bimódulo dada pelas multiplicações à direita e à esquerda.

Observe que um $A$-bimódulo $M$ pode ser identificado com um $A^{e}$-módulo à esquerda definindo

$$
(a \otimes b) \cdot m=a m b,
$$

também podemos identificar $M$ com um $A^{e}$-módulo à direita definindo

$$
m \cdot(a \otimes b)=b m a .
$$

A partir de agora quando nos referirmos a um módulo estaremos querendo dizer módulo à esquerda caso não seja especificado o contrário.

Note que a álgebra $A$ é um $A^{e}$-módulo à esquerda ou à direita, de fato se $A^{\otimes n}=A \otimes \cdots \otimes A$ temos que

$$
(a \otimes b) \cdot\left(c_{1} \otimes c_{2} \otimes \cdots \otimes c_{n-1} \otimes c_{n}\right)=a c_{1} \otimes c_{2} \otimes \cdots \otimes c_{n-1} \otimes c_{n} b
$$

para todo $a, b, c_{1}, \ldots, c_{n} \in A$ define uma estrutura de $A^{e}$-módulo em $A^{\otimes n}$.

Considere a seguinte sequência de $A$-bimódulos:

$$
\cdots \stackrel{d_{3}}{\longrightarrow} A^{\otimes 4} \stackrel{d_{2}}{\longrightarrow} A^{\otimes 3} \stackrel{d_{1}}{\longrightarrow} A \otimes A \stackrel{\pi}{\longrightarrow} A \longrightarrow 0
$$

onde $\pi$ é a multiplicação, $d_{1}(a \otimes b \otimes c)=a b \otimes c-a \otimes b c$ para $a, b, c \in A$ e em geral

$$
d_{n}\left(a_{0} \otimes \cdots \otimes a_{n+1}\right)=\sum_{i=0}^{n}(-1)^{i} a_{0} \otimes \cdots \otimes a_{i} a_{i+1} \otimes \cdots \otimes a_{n+1}
$$


para todos $a_{0}, \ldots, a_{n+1}$ em $A$. Pode-se verificar diretamente que esta sequencia é um complexo de $A$-bimódulos, isto é, $d_{n-1} d_{n}=0$ para todo $n$.

Usaremos o seguinte lema para mostrar que o complexo 3.0 é exato.

Lema 3.1.1. Considere o seguinte complexo

$$
\cdots \stackrel{d_{k+2}}{\longrightarrow} C_{k+1} \stackrel{d_{k+1}}{\longrightarrow} C_{k} \stackrel{d_{k}}{\longrightarrow} C_{k-1} \stackrel{d_{k-1}}{\longrightarrow} \cdots
$$

Agora suponha que exista uma contração do complexo, isto é, um conjunto de morfismos

$$
s_{n}: C_{n} \rightarrow C_{n+1}
$$

tais que

$$
d_{n+1} s_{n}+s_{n-1} d_{n}=i d .
$$

Então o complexo é exato.

Demonstração. Seja $a \in \operatorname{ker} d_{n-1}$. Temos $a=d_{n} s_{n-1}(a)+s_{n-2} d_{n-1}(a)=d_{n}\left(s_{n-1}(a)\right)$, portanto $\operatorname{Im} d_{n}=\operatorname{ker} d_{n-1}$

Agora tome a contração do complexo 3.0 dada por

$$
s_{n}\left(a_{0} \otimes \cdots \otimes a_{n+1}\right)=1 \otimes a_{0} \otimes \cdots \otimes a_{n+1}
$$

para todo $n$ e todos $a_{0}, \ldots, a_{n+1} \in A$. Observe que $s_{n}$ é de fato uma contração pois

$$
\begin{aligned}
d_{n+1} s_{n}\left(a_{o} \otimes \cdots \otimes a_{n+1}\right) & =d_{n+1}\left(1 \otimes a_{o} \otimes \cdots \otimes a_{n+1}\right) \\
& =a_{o} \otimes \cdots \otimes a_{n+1}-\sum_{i=0}^{n}(-1)^{i} 1 \otimes a_{0} \otimes \cdots \otimes a_{i} a_{i+1} \otimes \cdots \otimes a_{n+1}
\end{aligned}
$$

$\mathrm{e}$

$$
\begin{aligned}
s_{n-1} d_{n}\left(a_{o} \otimes \cdots \otimes a_{n+1}\right) & =s_{n-1}\left(\sum_{i=0}^{n}(-1)^{i} a_{0} \otimes \cdots \otimes a_{i} a_{i+1} \otimes \cdots \otimes a_{n+1}\right) \\
& =\sum_{i=0}^{n}(-1)^{i} 1 \otimes a_{0} \otimes \cdots \otimes a_{i} a_{i+1} \otimes \cdots \otimes a_{n+1} .
\end{aligned}
$$

Portanto $d_{n+1} s_{n}+s_{n-1} d_{n}=i d$ para todo $n$ e o complexo $3 . \rrbracket$ é exato.

Definimos $B_{n}(A):=A^{\otimes n+2}$ e chamaremos o complexo truncado

$$
B(A): \cdots \stackrel{d_{3}}{\longrightarrow} A^{\otimes 4} \stackrel{d_{2}}{\longrightarrow} A^{\otimes 3} \stackrel{d_{1}}{\longrightarrow} A \otimes A \longrightarrow 0
$$

o complexo bar associado ao $A^{e}$-módulo $A$.

Note que a homologia do complexo [.2. está concentrada em grau 0, onde ela é simplesmente A.

Ocasionalmente usaremos o isomorfismo de $A^{e}$-módulos à esquerda

$$
A^{\otimes(n+2)} \stackrel{\sim}{\rightarrow} A^{e} \otimes A^{n}
$$

dado por

$$
a_{0} \otimes a_{1} \otimes \cdots \otimes a_{n} \otimes a_{n+1} \mapsto\left(a_{0} \otimes a_{n+1}\right) \otimes\left(a_{1} \otimes \cdots \otimes a_{n}\right),
$$

para todo $a_{0}, \ldots, a_{n+1} \in A$, onde a ação de $A^{e}$ em $A^{e} \otimes A^{n}$ é dada pela multiplicação no fator à esquerda. 
Seja $M$ um $A$-bimódulo e considere o complexo

$$
M \otimes_{A^{e}} B(A)
$$

de $k$-espaços vetoriais com os diferenciais $1_{M} \otimes d_{n}$. Usaremos o isomorfismo de $k$-espaços vetoriais

$$
M \otimes_{A^{e}} B_{n}(A) \stackrel{\sim}{\rightarrow} M \otimes A^{\otimes n}
$$

dado por

$$
m \otimes_{A^{e}} a_{0} \otimes \cdots \otimes a_{n+1} \mapsto a_{n+1} m a_{0} \otimes a_{1} \otimes \cdots \otimes a_{n},
$$

para todos $m \in M$ e $a_{0}, \ldots, a_{n+1} \in A$. Com esse isomorfismo temos que o diferencial em $M \otimes A^{\otimes n}$ que corresponde a $1_{M} \otimes_{A^{e}} d_{n}$ em $M \otimes_{A^{e}} A^{\otimes(n+2)}$ é

$$
\begin{aligned}
\delta_{n}\left(m \otimes a_{1} \otimes a_{2} \otimes \cdots \otimes a_{n}\right) & =m a_{1} \otimes a_{2} \otimes \cdots \otimes a_{n} \\
& +\sum_{i=1}^{n-1}(-1)^{i} m \otimes a_{1} \otimes a_{2} \otimes \cdots \otimes a_{i} a_{i+1} \otimes \cdots \otimes a_{n} \\
& +(-1)^{n} a_{n} m \otimes a_{1} \otimes a_{2} \otimes \cdots \otimes a_{n-1} .
\end{aligned}
$$

Definição 3.1.1. A homologia de Hochschild $H H_{*}(A, M)$ de $A$ com coeficientes em um $A$ bimódulo $M$ é a homologia do complexo B.3. Equivalentemente

$$
H H_{n}(A, M)=H_{n}\left(M \otimes A^{\otimes \cdot}\right),
$$

isto é, $H H_{n}(A, M)=\operatorname{ker}\left(\delta_{n}\right) / \operatorname{Im}\left(\delta_{n+1}\right)$ para todo $n \geq 0$. Defina $H H_{*}(A, M)=\oplus_{n \geq 0} H H_{n}(A, M)$.

Agora considere o complexo

$$
\operatorname{Hom}_{A^{e}}(B(A), M)
$$

de $k$-módulos com os diferenciais $d_{n}^{*}$, onde $d_{n}^{*}(f)=f d_{n}$ para toda $f \in \operatorname{Hom}\left(A^{\otimes(n+2)}, M\right)$. Observe que temos o seguinte isomorfismo de $k$-módulos

$$
\operatorname{Hom}_{A^{e}}\left(B_{n}(A), M\right) \stackrel{\sim}{\rightarrow} \operatorname{Hom}_{k}\left(A^{\otimes n}, M\right)
$$

dado por

$$
g \mapsto\left(a_{1} \otimes \ldots \otimes a_{n} \mapsto g\left(1 \otimes a_{1} \otimes \cdots \otimes a_{n} \otimes 1\right)\right)
$$

para toda $g \in \operatorname{Hom}_{A^{e}}\left(B_{n}(A), M\right)$ e $a_{1}, \ldots, a_{n} \in A$.

Usando este isomorfismo temos que o diferencial em $\operatorname{Hom}_{k}\left(A^{\otimes n}, M\right)$ que corresponde a $d_{n}^{*}$ em $\operatorname{Hom}_{A^{e}}\left(B_{n}(A), M\right)$ é

$$
\begin{aligned}
\partial_{n}(f)\left(a_{1} \otimes \cdots \otimes a_{n}\right) & =a_{1} f\left(a_{2} \otimes \cdots \otimes a_{n}\right) \\
& +\sum_{i=1}^{n-1}(-1)^{i} f\left(a_{1} \otimes \cdots \otimes a_{i} a_{i+1} \otimes \cdots \otimes a_{n}\right) \\
& +(-1)^{n} f\left(a_{1} \otimes \cdots \otimes a_{n-1}\right) a_{n}
\end{aligned}
$$

Definição 3.1.2. A Cohomologia de Hochschild $H H^{*}(A, M)$ de $A$ com coeficientes no $A$ bimódulo $M$ é a cohomologia do complexo 3.4, equivalentemente

$$
H H^{n}(A, M)=H^{n}\left(\operatorname{Hom}_{k}\left(A^{\otimes \cdot}, M\right)\right),
$$

isto é, $H H^{n}(A, M)=\operatorname{ker}\left(\partial_{n+1}\right) / \operatorname{Im}\left(\partial_{n}\right)$ para todo $n \geq 0$. 
Quando tomamos $M=A$ usaremos a seguinte notação

$$
H H_{*}(A)=H H_{*}(A, A) \text { e } H H^{*}(A)=H H^{*}(A, A) .
$$

A proposição a seguir permite calcularmos a homologia e cohomologia de Hochschild usando outras resoluções da álgebra $A$ além do complexo bar. A vantagem disto é que dependendo da álgebra $A$ existem resoluções projetivas de $A$ como $A^{e}$-módulo que são mais convenientes que o complexo bar, que é muito grande.

Proposição 3.1.2. Seja $M$ um A-bimódulo, então

$$
H H_{*}(A, M) \cong \operatorname{Tor}_{n}^{A^{e}}(A, M) \text { e } H H^{*}(A, M) \cong \operatorname{Ext}_{n}^{A^{e}}(A, M) .
$$

Demonstração. Mostraremos apenas o isomorfismo $H H_{*}(A, M) \cong \operatorname{Tor}_{n}^{A^{e}}(A, M)$, a demonstração do outro é análoga.

Como $k$ é um corpo temos que todo $k$-módulo é livre, em particular $A^{\otimes n}$ é um $k$-módulo livre e $A^{\otimes(n+2)} \cong A^{e} \otimes A^{\otimes n}$ é um $A^{e}$-módulo livre. Logo o complexo bar é uma resolução livre de $A$ como um $A^{e}$-módulo.

Tomando o produto tensorial desta resolução livre com $M$ nos dá o complexo de Hochschild e portanto temos $H H_{*}(A, M) \cong \operatorname{Tor}_{n}^{A^{e}}(A, M)$.

O exemplo a seguir nos mostrará como o fato de podermos usar outras resoluções pode ajudar a calcular a cohomologia de Hochschild.

Exemplo 3.1.2. Seja $A=k[x]$. Considere a seguinte sequência

$$
0 \longrightarrow k[x] \otimes k[x] \stackrel{x \otimes 1-1 \otimes x}{\longrightarrow} k[x] \otimes k[x] \stackrel{\pi}{\longrightarrow} k[x] \longrightarrow 0
$$

onde $\pi$ é a multiplicação e $(x \otimes 1-1 \otimes x)$ é a multiplicação pelo elemento $x \otimes 1-1 \otimes x$.

Note que este complexo é isomorfo ao seguinte complexo

$$
0 \longrightarrow k[x, y] \stackrel{\epsilon}{\longrightarrow} k[x, y] \stackrel{\delta}{\longrightarrow} k[x] \longrightarrow 0
$$

onde $\epsilon(f(x, y))=(x-y) f(x, y)$ e $\delta(f(x, y))=f(x, x)$, e portanto [.] é uma resolução livre de $k[x]$.

Aplicando o funtor $\operatorname{Hom}_{k[x]^{e}}(-, k[x])$ na sequência obtida deletando o termo $k[x]$ em $[.6]$ obtemos o complexo

$$
0 \longleftarrow \operatorname{Hom}_{k[x]^{e}}(k[x] \otimes k[x], k[x]) \longleftarrow \operatorname{Hom}_{k[x]}(k[x] \otimes k[x], k[x]) \longleftarrow
$$

Tomando o isomorfismo $\operatorname{Hom}_{k[x]^{e}}(k[x] \otimes k[x], k[x]) \rightarrow k[x]$ dado por $f \mapsto f(1 \otimes 1)$ o complexo obtemos o complexo

$$
0 \longleftarrow k[x] \longleftarrow \psi k[x] \longleftarrow 0
$$

Agora basta calcularmos qual é o morfismo $\psi$. Seja $a \in k[x]$, identificado com a função $f_{a} \in$ $\operatorname{Hom}_{k[x]^{e}}(k[x] \otimes k[x], k[x])$, onde $f_{a}(1 \otimes 1)=a$. Compondo com $(x \otimes 1-1 \otimes x)$ temos

$$
f_{a}((x \otimes 1-1 \otimes x)(1 \otimes 1))=x f_{a}(1 \otimes 1)-f_{a}(1 \otimes 1) x=x a-a x=0,
$$

pois $k[x]$ é comutativo. Portanto $\psi=0 \mathrm{e}$

$$
H H^{0}(k[x]) \cong k[x], H H^{1}(k[x]) \cong k[x] \text { e } H H^{n}(k[x])=0
$$

para $n \geq 2$. 


\subsection{Interpretação em Graus Baixos}

As definições de homologia e cohomologia de Hochschild através do complexo bar nos permitem as seguintes observações em graus baixos.

Grau 0: Por definição temos $H H^{0}(A, M)=\operatorname{ker}\left(d_{1}^{*}\right)$. Suponha que $f \in \operatorname{Hom}_{A^{e}}(A \otimes A, M)$ é tal que $d_{1}^{*}(f)=0$, isto é, para cada $a \in A$ temos

$$
0=d_{1}^{*}(f)(1 \otimes a \otimes 1)=f\left(d_{1}(1 \otimes a \otimes 1)\right)=f(a \otimes 1)-f(1 \otimes a)=a f(1 \otimes 1)-f(1 \otimes 1) a .
$$

Portanto $f(1 \otimes 1)$ é um elemento $m \in M$ tal que $a m=m a$ para todo $a \in A$ e $f$ é determinada por esse elemento $m$ pois $f(b \otimes c)=b f(1 \otimes 1) c=b m c$ para todo $b, c \in A$. Reciprocamente, dado um $m \in M$ tal que $a m=m a$ para todo $a \in A$ podemos definir $f_{m} \in \operatorname{Hom}_{A^{e}}(A \otimes A, M)$ por $f_{m}(b \otimes c)=b m c$ para todo $b, c \in A$, temos que vale $d_{1}^{*}\left(f_{m}\right)=0$. Logo, como um espaço vetorial,

$$
H H^{0}(A, M) \cong\{m \in M ; a m=m a \text { para todo } a \in A\} .
$$

Observe que se $M=A$ então $H H^{0}(A) \cong Z(A)$, o centro de $A$. Note que isto está de acordo com o que calculamos no caso $A=k[x]$.

Grau 1: Por definição $H H^{1}(A, M)=\operatorname{ker}\left(d_{2}^{*}\right) / \operatorname{Im}\left(d_{1}^{*}\right)$. Seja $f \in \operatorname{ker}\left(d_{2}^{*}\right)$, isto é, $f \in \operatorname{Hom}_{A^{e}}\left(A^{\otimes 3}, M\right)$ e

$$
\begin{aligned}
0= & d_{2}^{*}(f)(1 \otimes a \otimes b \otimes 1) \\
& =f(a \otimes b \otimes 1-1 \otimes a b \otimes 1+1 \otimes a \otimes b) \\
& =a f(1 \otimes b \otimes 1)-f(1 \otimes a b \otimes 1)+f(1 \otimes a \otimes 1) b .
\end{aligned}
$$

Escrevendo $f$ para a imagem de $f$ sob o isomorfismo $\operatorname{Hom}_{A^{e}}\left(A^{\otimes 3}, M\right) \stackrel{\sim}{\rightarrow} \operatorname{Hom}_{k}(A, M)$ dado por $f \mapsto(a \mapsto f(1 \otimes a \otimes 1)$, temos que a equação acima fica

$$
0=a f(b)-f(a b)+f(a) b,
$$

$\mathrm{ou}$

$$
f(a b)=a f(b)+f(a) b
$$

para todos $a, b \in A$, que é a condição para $f$ ser uma $k$-derivação.

Suponha agora que $f \in \operatorname{Im}\left(d_{1}^{*}\right)$, isto é, $f=d_{1}^{*}(g)$ para alguma $g \in \operatorname{Hom}_{A^{e}}\left(A^{\otimes 2}, M\right)$. Como no exemplo anterior temos que $g$ é dada por seu valor em $1 \otimes 1$, seja $m=g(1 \otimes 1)$. Então

$$
\begin{aligned}
d_{1}^{*}(g)(1 \otimes a \otimes 1) & =g(a \otimes 1-1 \otimes a) \\
& =a g(1 \otimes 1)-g(1 \otimes 1) a=a m-m a .
\end{aligned}
$$

Logo $d_{1}^{*}(g)$ é a $k$-derivação interna dada por $m \in M$, e toda derivação interna é dada por um elemento de $\operatorname{Im}\left(d_{1}^{*}\right)$.

Denotando o espaço das $k$-derivações de $A$ para $M$ por

$$
\operatorname{Der}(A, M)
$$

e o espaço das $k$-derivações internas de $A$ para $M$ por

$$
\operatorname{InnDer}(A, M)
$$

temos

$$
H H^{1}(A, M) \cong \operatorname{Der}(A, M) / \operatorname{InnDer}(A, M) \text {. }
$$




\subsection{Produto Cup}

Nesta seção veremos que $H H^{*}(A)$ além de ser um espaço vetorial graduado, também possui um produto associativo que lhe dá a estrutura de uma álgebra graduada. Definiremos este produto no nivel de cadeias usando o complexo bar.

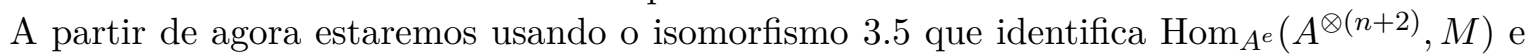
$\operatorname{Hom}_{k}\left(A^{\otimes n}, M\right)$. Seja

$$
C^{*}(A, M)=\bigoplus_{n \geq 0} \operatorname{Hom}_{k}\left(A^{\otimes n}, M\right),
$$

o espaço das cocadeias de Hochschild em A com coeficientes em M.

Definição 3.3.1. Sejam $f \in \operatorname{Hom}_{k}\left(A^{\otimes m}, M\right)$ e $g \in \operatorname{Hom}_{k}\left(A^{\otimes n}, M\right)$. O produto cup $f \smile g$ é o elemento de $\operatorname{Hom}_{k}\left(A^{\otimes(m+n)}, M\right)$ dado por

$$
(f \smile g)\left(a_{1} \otimes \cdots \otimes a_{m+n}\right)=f\left(a_{1} \otimes \cdots \otimes a_{m}\right) g\left(a_{m+1} \otimes \cdots \otimes a_{m+n}\right)
$$

para todos $a_{1}, \ldots, a_{m+n} \in A$. Se $m=0$ devemos interpretar essa fórmula da seguinte maneira

$$
(f \smile g)\left(a_{1} \otimes \cdots \otimes a_{n}\right)=f(1) g\left(a_{1} \otimes \cdots \otimes a_{n}\right)
$$

e análogamente caso $n=0$.

Da definição segue que o produto cup é associativo em cadeias e segue também o seguinte lema

\section{Lema 3.3.1.}

$$
d_{m+n+1}^{*}(f \smile g)=\left(d_{m+1}^{*}(f)\right) \smile g+(-1)^{m} f \smile\left(d_{n+1}(g)\right) .
$$

Deste lema segue que o produto cup definido em cadeias induz um produto bem definido ao nível de cohomologia que também chamaremos de produto cup e usaremos a mesma notação

$$
\smile: H H^{m}(A) \times H H^{n}(A) \rightarrow H H^{m+n}(A) .
$$

\subsection{Colchete de Gerstenhaber}

Além do produto cup, o anel de cohomologia de Hochschild $H H^{*}(A)$ possui outra operação que é uma derivação graduada com respeito ao produto cup. Definiremos este produto ao nível de cocadeias utilizando o complexo bar.

Nesta seção suponha que $k$ é um corpo e $A$ é uma álgebra sobre este corpo.

Definição 3.4.1. Sejam $f \in H_{o m}\left(A^{\otimes m}, A\right)$ e $g \in H o m_{k}\left(A^{\otimes n}, A\right)$. O colchete de Gerstenhaber $\llbracket f, g \rrbracket$ é definido como o elemento de $H_{o} m_{k}\left(A^{\otimes(m+n-1)}, A\right)$ dado por

$$
\llbracket f, g \rrbracket=f \circ g-(-1)^{(m-1)(n-1)} g \circ f
$$

onde $f \circ g$ é definido como

$f \circ g\left(a_{1} \otimes \cdots \otimes a_{m+n-1}\right)=\sum_{i=1}^{m}(-1)^{j} f\left(a_{1} \otimes \cdots \otimes a_{i-1} \otimes g\left(a_{i} \otimes \cdots \otimes a_{i+n-1}\right) \otimes a_{i+n} \otimes \cdots \otimes a_{m+n-1}\right)$,

onde $j=(i-1)(n-1)$. Se $m=0$ temos $f \circ g=0$ pois a soma será vazia e se $n=0$ a fórmula deve ser interpretada tomando o valor $g(1)$ no lugar de $g\left(a_{i} \otimes \cdots \otimes a_{i+n-1}\right)$.

Os lemas a seguir nos dão algumas propriedades que o colchete de Gerstenhaber satisfaz, todas as demonstrações podem ser feitas calculando diretamente no complexo bar. 
Lema 3.4.1. Sejam $f \in \operatorname{Hom}_{k}\left(A^{\otimes m}, A\right), g \in \operatorname{Hom}_{k}\left(A^{\otimes n}, A\right)$ e $h \in H_{o m}\left(A^{\otimes p}, A\right)$. Então valem as seguintes propriedades

1. $\llbracket f, g \rrbracket=-(-1)^{(m-1)(n-1)} \llbracket g, f \rrbracket$,

2. $(-1)^{(m-1)(p-1)} \llbracket f, \llbracket g, h \rrbracket \rrbracket+(-1)^{(n-1)(m-1)} \llbracket g, \llbracket h, f \rrbracket \rrbracket+(-1)^{(p-1)(n-1)} \llbracket h, \llbracket f, g \rrbracket \rrbracket=0$,

3. $d^{*}(\llbracket f, g \rrbracket)=(-1)^{(n-1)} \llbracket d^{*} f, g \rrbracket+\llbracket f, d^{*} g \rrbracket$.

Lema 3.4.2. Sejam $\pi: A \otimes A \rightarrow A$ a multiplicação, $f \in H_{k}\left(A^{\otimes m}, A\right)$ e $g \in H_{o} m_{k}\left(A^{\otimes n}, A\right)$. Então

1. $f \smile g-(-1)^{m n} g \smile f=\left(d^{*} g\right) \circ f+(-1)^{m} d^{*}(g \circ f)+(-1)^{m-1} g \circ\left(d^{*} f\right)$,

2. $[f, \pi]=-d^{*}(f)$.

Como consequência desses dois lemas temos os seguintes fatos

Teorema 3.4.3. $\quad$ 1. O produto cup é comutativo graduado em $H H^{*}(A)$,

2. O colchete de Gerstenhaber induz um colchete em $H H^{*}(A)$ que também chamaremos de colchete de Gerstenhaber

Demonstração. 1 segue do lema [3.4.2(1) observando que o lado direito da igualdade vale zero em cohomologia. 2 segue diretamente do lema 3.4.D(3).

O lema a seguir nos dá outra propriedade que o colchete de Gerstenhaber satisfaz a nivel de cohomologia, uma demonstração se encontra em (Gerstenhaber, 1963), onde é mostrado que a diferença dos lados esquerdo e direito da equação é igual a um certo cobordo.

Lema 3.4.4. Sejam $U \in H H^{u}(A), V \in H H^{v}(A)$ e $W \in H H^{w}(A)$. Então

$$
\llbracket U \smile V, W \rrbracket=\llbracket U, W \rrbracket \smile V+(-1)^{u(w-1)} U \smile \llbracket V, W \rrbracket
$$

\subsection{Versão Topológica}

Para aplicações da cohomologia de Hochschild em geometria será necessário que consideremos álgebras topológicas, bimódulos topológicos, cadeias e cocadeias contínuas e resoluções topológicas. De fato, enquanto os grupos de cohomologia algébrica de Hochschild da álgebra de funções suaves em uma variedade suave não foram calculados, os grupos de cohomologia contínua de Hochschild podem ser calculados.

A seguir faremos uma breve adaptação do conteúdo da seção anterior ao caso contínuo, seguindo (Connes, 11994). A classe correta de álgebras topológicas que nos dão uma teoria interessante é a classe de álgebras localmente convexas.

Assumiremos nesta seção que $A$ é uma álgebra localmente convexa tal que a multiplicação $A \times A \rightarrow A$ é contínua. Isto é, para cada seminorma contínua $\rho$ em $A$ existe uma seminorma contínua $\rho^{\prime}$ tal que $\rho(a b) \leq \rho^{\prime}(a) \rho^{\prime}(b)$ para $a, b \in A$.

Definição 3.5.1. Seja $A$ uma álgebra localmente convexa. Diremos que $M$ é um $A$-módulo topológico se $M$ é um espaço vetorial localmente convexo que também é um $A$-módulo tal que a multiplicação $(a, m) \mapsto a m$ é contínua.

Dizemos que uma álgebra localmente convexa $A$ é de Fréchet se sua topologia é metrizável e completa. Muitos exemplos que iremos encontrar são álgebras de Fréchet, como a álgebra de funções suaves em uma variedade.

Dados dois espaços vetoriais localmente convexos $V_{1}$ e $V_{2}$, o produto tensorial projetivo de $V_{1}$ e $V_{2}$ é o espaço vetorial localmente convexo $V_{1} \widehat{\otimes} V_{2}$ com uma aplicação contínua bilinear $V_{1} \times V_{2} \rightarrow V_{1} \widehat{\otimes} V_{2}$ que é universal no seguinte sentido, para cada espaço localmente convexo $W$ existe um isomorfismo entre aplicações bilineares contínuas $V_{1} \times V_{2} \rightarrow W$ e aplicações lineares contínuas $V_{1} \widehat{\otimes} V_{2} \rightarrow W$. 
Definição 3.5.2. Diremos que um $A$-módulo topológico $M$ é projetivo se existe um $A$-módulo topológico $N$ tal que $M \oplus N=A \widehat{\otimes} E$, onde $E$ é um espaço vetorial localmente convexo completo.

Para calcular a cohomologia de Hochschild no caso topológico há uma pequena diferença do caso algébrico no uso de resoluções projetivas. Para mostrar que a cohomologia independe da resolução usada precisaremos que as resoluções possuam uma contração contínua.

Usaremos a notação $A^{e}=A \widehat{\otimes} A^{o p}$.

Definição 3.5.3. Seja $A$ uma álgebra localmente convexa sobre $\mathbb{C}$ e $M$ um $A^{e}$-módulo topológico. Uma resolução projetiva topológica de $M$ é uma sequência exata de $A^{e}$-módulos projetivos e aplicações $A^{e}$-lineares contínuas

$$
\cdots \stackrel{b_{3}}{\longrightarrow} M_{2} \stackrel{b_{2}}{\longrightarrow} M_{1} \stackrel{b_{1}}{\rightarrow} M_{0} \stackrel{\epsilon}{\rightarrow} M
$$

que admite uma homotopia $\mathbb{C}$-linear contínua $s_{i}: M_{i} \rightarrow M_{i+1}$

$$
b_{i+1} s_{i}+s_{i-1} b_{i}=i d, \forall i .
$$

Definição 3.5.4. Seja $A$ uma álgebra localmente convexa sobre $\mathbb{C}$ e $M$ um $A^{e}$-módulo topológico. Seja

$$
\cdots \stackrel{b_{3}}{\longrightarrow} M_{2} \stackrel{b_{2}}{\longrightarrow} M_{1} \stackrel{b_{1}}{\longrightarrow} M_{0} \stackrel{\epsilon}{\longrightarrow} A
$$

uma resolução projetiva topológica de $A$.

A cohomologia de Hochschild contínua de $A$ com coeficientes em $M, H_{c}^{*}(A, M)$ é a cohomologia do complexo

$$
\operatorname{Hom}_{A^{e}}\left(M_{0}, M\right) \stackrel{b_{1}^{*}}{\longrightarrow} \operatorname{Hom}_{A^{e}}\left(M_{1}, M\right) \stackrel{b_{2}^{*}}{\longrightarrow} \operatorname{Hom}_{A^{e}}\left(M_{2}, M\right) \stackrel{b_{3}^{*}}{\longrightarrow} \cdots
$$

onde $\operatorname{Hom}_{A^{e}}$ quer dizer aplicações $A^{e}$-lineares e contínuas.

Observe que para esta definição fazer sentido é necessário que os grupos de cohomologia não dependam da resolução escolhida. De fato, em (Connes, 1994) temos o seguinte lema

Lema 3.5.1. Dadas duas resoluções projetivas topológicas $\left(M_{n}, b_{n}\right)$ e $N_{n}, c_{n}$ do módulo A sobre $A^{e}$ e um $A^{e}$-módulo $M$ temos que os grupos de cohomologia dos complexos

$$
\operatorname{Hom}_{A^{e}}\left(M_{0}, M\right) \stackrel{b_{1}^{*}}{\longrightarrow} \operatorname{Hom}_{A^{e}}\left(M_{1}, M\right) \stackrel{b_{2}^{*}}{\longrightarrow} \operatorname{Hom}_{A^{e}}\left(M_{2}, M\right) \stackrel{b_{3}^{*}}{\longrightarrow} \cdots
$$

$e$

$$
\operatorname{Hom}_{A^{e}}\left(N_{0}, M\right) \stackrel{c_{1}^{*}}{\longrightarrow} \operatorname{Hom}_{A^{e}}\left(N_{1}, M\right) \stackrel{c_{2}^{*}}{\longrightarrow} \operatorname{Hom}_{A^{e}}\left(N_{2}, M\right) \stackrel{c_{3}^{*}}{\longrightarrow} \cdots
$$

são isomorfos.

Exemplo 3.5.1. Observe que se $A$ é unital temos a seguinte resolução projetiva topológica.

- $M_{n}=A^{e} \widehat{\otimes} E_{n}$, com $E_{n}=\underbrace{A \widehat{\otimes} \cdots \widehat{\otimes} A}_{n \text { fatores }}$

- $\epsilon: M_{0} \rightarrow A$ é dado por $\epsilon(a \otimes b)=a b, \forall a, b \in A$

$$
\begin{aligned}
b_{n}\left((1 \otimes 1) \otimes\left(a_{1} \otimes \cdots \otimes a_{n}\right)\right) & =\left(a_{1} \otimes 1\right) \otimes\left(a_{2} \otimes \cdots \otimes a_{n}\right) \\
& +\sum_{j=1}^{n-1}(-1)^{j}(1 \otimes 1) \otimes\left(a_{1} \otimes \cdots \otimes a_{i} a_{i+1} \otimes \cdots \otimes a_{n}\right) \\
& +(-1)^{n}\left(1 \otimes a_{n}\right) \otimes\left(a_{1} \otimes \cdots \otimes a_{n-1}\right)
\end{aligned}
$$




$$
\text { - } s_{n}\left((a \otimes b) \otimes\left(a_{1} \otimes \cdots \otimes a_{n}\right)\right)=(1 \otimes b) \otimes\left(a \otimes a_{1} \otimes \cdots \otimes a_{n}\right)
$$

Exemplo 3.5.2. Seja $A=C^{\infty}\left(S^{1}\right)$ a álgebra de funções complexas no círculo $S^{1}$. Observe que $C^{\infty}\left(S^{1}\right) \widehat{\otimes} C^{\infty}\left(S^{1}\right)^{o p} \cong C^{\infty}\left(S^{1}\right) \widehat{\otimes} C^{\infty}\left(S^{1}\right)$. A é uma álgebra localmente convexa e temos a seguinte resolução topológica de $C^{\infty}\left(S^{1}\right)$

$$
0 \longleftarrow C^{\infty}\left(S^{1}\right) \overleftarrow{\epsilon} C^{\infty}\left(S^{1}\right) \widehat{\otimes} C^{\infty}\left(S^{1}\right) \overleftarrow{d} C^{\infty}\left(S^{1}\right) \widehat{\otimes} C^{\infty}\left(S^{1}\right) \otimes \mathbb{C} \longleftarrow 0
$$

onde $\epsilon(1 \otimes 1)=1$ e $d(1 \otimes 1 \otimes 1)=x \otimes 1-1 \otimes x$. Para verificar a exatidão deste complexo primeiramente observe que é trivial que $\operatorname{Im} d \subset \operatorname{ker} \epsilon$, $\log$ basta verificar que $\operatorname{ker} \epsilon \subset \operatorname{Im} d$. Note que

$$
C^{\infty}\left(S^{1}\right) \widehat{\otimes} C^{\infty}\left(S^{1}\right) \cong C^{\infty}\left(S^{1} \times S^{1}\right) .
$$

Usando este isomorfismo obtemos o complexo

$$
0 \longleftarrow C^{\infty}\left(S^{1}\right) \overleftarrow{\epsilon} C^{\infty}\left(S^{1} \times S^{1}\right) \overleftarrow{d} C^{\infty}\left(S^{1} \times S^{1}\right) \longleftarrow 0
$$

onde

$$
(\epsilon f)(x)=f(x, x) \quad \text { e } \quad(d f)(x, y)=(x-y) f(x, y) .
$$

A fórmula

$$
f(x, y)=f(x, x)-(x-y) \int_{0}^{1} \frac{\partial}{\partial y} f(x, y+t(x-y)) \mathrm{dt}
$$

nos mostra que $\operatorname{ker} \epsilon \subset \operatorname{Im} d$.

Aplicando o funtor $\operatorname{Hom}_{A \widehat{\otimes} A}(\cdot, A)$ à resolução anterior, onde estamos considerando apenas homomorfismos contínuos, obtemos o complexo

$$
0 \longrightarrow \operatorname{Hom}_{A \widehat{\otimes} A}\left(C^{\infty}\left(S^{1} \times S^{1}\right), A\right) \stackrel{d^{*}}{\rightarrow} \operatorname{Hom}_{A \widehat{\otimes} A}\left(C^{\infty}\left(S^{1} \times S^{1}\right), A\right) \longrightarrow 0,
$$

que é isomorfo ao complexo

$$
0 \longrightarrow C^{\infty}\left(S^{1}\right) \stackrel{d^{*}}{\rightarrow} C^{\infty}\left(S^{1}\right) \longrightarrow 0 .
$$

É facíl ver que neste complexo $d^{*}=0$ e portanto temos

$$
H H^{0}\left(C^{\infty}\left(S^{1}\right)\right)=C^{\infty}\left(S^{1}\right), H H^{1}\left(C^{\infty}\left(S^{1}\right)\right)=C^{\infty}\left(S^{1}\right) \text { e } H H^{i}\left(C^{\infty}\left(S^{1}\right)\right)=0 \text {, para } i \neq 0,1 .
$$

\subsection{Equivalência de Morita}

Com suas origens na teoria de representações de álgebras, a teoria de Morita também é útil em geometria não comutativa. As álgebras Morita equivalentes possuem $K$-teoria, cohomologia de Hochschild e cohomologia cíclica isomorfas. Além disso, para quocientes bons, a álgebra de funções no quociente é Morita equivalente ao quociente não comutativo.

Nesta seção toda álgebra $A$ será associativa e unital sobre $\mathbb{C}$. Todos os módulos serão unitários, isto é, a unidade de $A$ agirá nos módulos como o operador unitário. Sejam $A$ e $B$ álgebras, denotaremos por $\mathcal{M}_{A},{ }_{A} \mathcal{M}$ e ${ }_{A} \mathcal{M}_{B}$ as categorias de $A$-módulos à direita, $A$-módulos à esquerda e $A-B$-bimódulos, respectivamente.

Assumiremos a seguir que as equivalências de categorias são dadas por funtores aditivos.

Definição 3.6.1. As álgebras $A$ e $B$ são ditas Morita equivalentes se existe uma equivalência de categorias

$$
\mathcal{M}_{A} \simeq \mathcal{M}_{B}
$$


Note que se existe um funtor aditivo $F: \mathcal{M}_{A} \rightarrow \mathcal{M}_{B}$ que é exato à direita e comuta com somas diretas arbitrárias então existe um único $A-B$-bimódulo $X(X=F(A))$, tal que

$$
F(M)=M \otimes_{A} X, \quad \text { para todo } M \in \mathcal{M}_{A} .
$$

A afirmação oposta é verdade, isto é, o funtor $F: \mathcal{M}_{A} \rightarrow \mathcal{M}_{B}$ dado por $F(M)=M \otimes_{A} X$, para algum $A-B$-bimódulo $X$, é um funtor aditivo, exato à direita e que comuta com somas diretas arbitrárias. Portanto temos o seguinte lema.

Lema 3.6.1. As álgebras $A$ e $B$ são Morita equivalentes se, e somente se, existe um $A-B$ bimódulo $X$ e um $B-A$-bimódulo $Y$ tal que temos isomorfismos de bimódulos

$$
X \otimes_{B} Y \cong A, \quad Y \otimes_{A} X \cong B,
$$

onde a estrutura de A-bimódulo em $A$ é dada por $a(b) c=a b c$ e análogamente para $B$.

Os bimódulos do Lema anterior são chamados bimódulos de equivalência. Segue do Lema que $A$ e $B$ são Morita equivalentes se, e somente se, temos uma equivalência de categorias

$$
{ }_{A} \mathcal{M} \simeq{ }_{B} \mathcal{M}
$$

e também que $A$ e $B$ são Morita equivalentes se, e somente se, temos uma equivalência de categorias

$$
{ }_{A} \mathcal{M}_{A} \simeq{ }_{B} \mathcal{M}_{B} .
$$

Exemplo 3.6.1. Toda álgebra $A$ é Morita equivalente à $M_{n}(A)$, a álgebra de matrizes $n \times n$ com coeficientes em $A$. Os $A-M_{n}(A)$-bimódulos de equivalência são dados por espaços de vetores linha e vetores coluna. Seja $X=A^{n}$ considerado como espaço de vetores linha com a ação óbvia à esquerda de $A$ e a ação à direita de $M_{n}(A)$ a multiplicação matrizes e seja $Y=A^{n}$ considerado como espaço de vetores coluna com a ação usual de $M_{n}(A)$ à esquerda e a ação usual de $A$ à direita.

As aplicações

$$
\left(a_{1}, \ldots, a_{n}\right) \otimes\left(b_{1}, \ldots, b_{n}\right) \mapsto \sum a_{i} b_{i}
$$

e

$$
\left(a_{1}, \ldots, a_{n}\right) \otimes\left(b_{1}, \ldots, b_{n}\right) \mapsto\left(a_{i} b_{j}\right)
$$

induzem os isomorfismos

$$
X \otimes_{M_{n}(A)} Y \cong A, \quad Y \otimes_{A} X \cong M_{n}(A),
$$

respectivamente.

Observe que este exemplo nos mostra que uma álgebra comutativa pode ser Morita equivalente à uma álgebra não comutativa.

Precisaremos do seguinte lema:

Lema 3.6.2. Suponha que ${ }_{A} X_{B}$ e ${ }_{B} Y_{A}$ são bimódulos induzindo uma equivalência de Morita entre $A$ e $B$. Então $X$ é um A-módulo à esquerda projetivo finitamente gerado e um B-módulo à direita finitamente gerado..

Demonstração. Como $Y \otimes_{A} X \cong B$ podemos escrever $1=y_{1} \otimes x_{1}+\cdots+y_{m} \otimes x_{m}$ para algum $m$. Defina $e: X \rightarrow A^{m}$ por $e(x)=\left(x \otimes y_{1}, \ldots, x \otimes y_{m}\right)$ e $h: A^{m} \rightarrow X$ por $h\left(a_{1}, \ldots, a_{m}\right)=\sum a_{i} x_{i}$. As aplicações e e $h$ são homomorfismos de $A$-módulos à esquerda. Como he $(x)=\sum\left(x \otimes y_{i}\right) x_{i}=$ $\sum x\left(y_{i} \otimes x_{i}\right)=x$. Logo $X$ é um somando direto de $A^{m}$ em $A$ - mod. A demonstração de que $P$ é um somando direto de $B^{n}$ em mod $-S$ é análoga. 
Suponha agora que $A$ e $B$ são álgebras Morita equivalentes com $A-B$-bimódulo de equivalência $X$ e $B-A$-bimódulo de equivalência $Y$. Defina o funtor $\Phi$ da categoria de $A$-bimódulos para a categoria de $B$-bimódulos por

$$
\Phi(M)=Y \otimes_{A} M \otimes_{A} X
$$

e, analogamente, defina o funtor $\Psi$ da categoria de $B$-bimódulos para a categoria de $A$-bimódulos por

$$
\Phi(M)=X \otimes_{B} M \otimes_{B} Y .
$$

Pelo lema anterior temos que $X$ e $Y$ são projetivos como $A$-módulos e $B$-módulos, respectivamente. Vemos facilmente que este funtor leva módulos projetivos em módulos projetivos, portanto $\Phi$ leva resoluções projetivas de $A$-bimódulos em resoluções projetivas de $B$-bimódulos e $\Psi$ leva resoluções projetivas de $B$-bimódulos em resoluções projetivas de $A$-bimódulos.

O seguinte teorema nos dá a invariância da cohomologia de Hochschild por equivalência de Morita.

Teorema 3.6.3. Suponha que ${ }_{A} X_{B} e_{B} Y_{A}$ são bimódulos induzindo uma equivalência de Morita entre $A$ e B. Então para cada A-bimódulo $M$ temos

$$
H H_{n}(A, M) \cong H H_{n}\left(Y \otimes_{A} M \otimes_{A} X\right) \quad \text { e } H H^{n}(A, M) \cong H H^{n}\left(Y \otimes_{A} M \otimes_{A} X\right) .
$$

Em particular, $H H_{n}(A) \cong H H_{n}(B)$ e $H H^{n}(A) \cong H H^{n}(B)$.

Demonstração. Temos o seguinte isomorfismo

$$
\begin{aligned}
\operatorname{Hom}_{A}\left(X \otimes_{B} \bullet, \bullet\right) \cong \operatorname{Hom}_{B}\left(Y \otimes_{A} X \otimes_{B} \bullet, Y \otimes_{A} \bullet\right) & \cong \operatorname{Hom}_{B}\left(B \otimes_{B} \bullet, Y \otimes_{A} \bullet\right) \\
& \cong \operatorname{Hom}_{B}\left(\bullet, Y \otimes_{A} \bullet\right) .
\end{aligned}
$$

Da mesma forma obtemos

$$
\operatorname{Hom}_{A}\left(\bullet, X \otimes_{B} \bullet\right) \cong \operatorname{Hom}_{B}\left(Y \otimes_{A} \bullet, \bullet\right) .
$$

E, portanto,

$$
\begin{aligned}
& \operatorname{Ext}_{A}^{n}\left(X \otimes_{B} \bullet, \bullet\right) \cong \operatorname{Ext}_{B}^{n}\left(\bullet, Y \otimes_{A} \bullet\right), \\
& \operatorname{Ext}_{A}^{n}\left(\bullet, X \otimes_{B} \bullet\right) \cong \operatorname{Ext}_{B}^{n}\left(Y \otimes_{A} \bullet, \bullet\right) .
\end{aligned}
$$

Logo

$$
\begin{aligned}
H H^{n}(A, M) & \cong \operatorname{Ext}_{A^{e}}^{n}(A, M) \cong \operatorname{Ext}_{A^{e}}^{n}\left(X \otimes_{B} Y, M\right) \\
& \cong \operatorname{Ext}_{B \otimes A^{o p}}^{n}\left(Y, Y \otimes_{A} M\right) \cong \operatorname{Ext}_{B^{e}}^{n}\left(B, Y \otimes_{A} M \otimes_{A} X\right) \\
& \cong H H^{n}\left(B, Y \otimes_{A} M \otimes_{A} X\right) .
\end{aligned}
$$

A demonstração dual desta, isto é, usando o funtor Tor, funciona para demonstrar o mesmo para homologia.

Exemplo 3.6.2. Seja $M$ uma variedade suave compacta na qual o grupo de Lie compacto $G$ age suavemente e a ação é livre.

Vamos mostrar que as álgebras $A=C^{\infty}(M) \rtimes_{\alpha} G=C^{\infty}\left(G, C^{\infty}(M)\right)$ e $B=C^{\infty}(M / G)$ são Morita equivalentes.

Tome $X=C^{\infty}(M)$ o $A-B$-bimódulo onde a ação de $B$ é dada pela multiplicação ponto a ponto, usando o isomorfismo $C^{\infty}(M / G) \cong C^{\infty}(M)^{G}$. A ação de $A$ em $X$ será dada pela seguinte fórmula

$$
\varphi \cdot f=\int_{G} \varphi(s) \alpha_{s} f \mathrm{~d} s
$$


para $\varphi \in A$ e $f \in C^{\infty}(M)$. Observe que

$$
\begin{aligned}
\varphi \cdot(\psi \cdot f)=\int_{G} \varphi(s) \alpha_{s}(\psi \cdot f) \mathrm{d} s & =\int_{G} \int_{G} \varphi(s) \alpha_{s}\left(\psi(r) \alpha_{r} f\right) \mathrm{d} s \mathrm{~d} r \\
& =\int_{G} \int_{G} \varphi(s) \alpha_{s} \psi(r) \alpha_{s r} f \mathrm{~d} s \mathrm{~d} r
\end{aligned}
$$

e

$$
\begin{aligned}
(\varphi \psi) \cdot f=\int_{G}(\varphi \psi)(r) \alpha_{r} f \mathrm{~d} r & =\int_{G} \int_{G} \varphi(s) \alpha_{s} \psi\left(s^{-1} r\right) \alpha_{r} f \mathrm{~d} s \mathrm{~d} r \\
& =\int_{G} \int_{G} \varphi(s) \alpha_{s} \psi(r) \alpha_{s r} f \mathrm{~d} s \mathrm{~d} r
\end{aligned}
$$

Portanto esta ação à esquerda está bem definida.

Vamos tomar $Y=C^{\infty}(M)$ o $B-A$-bimódulo oposto de $X$, isto é, a ação à esquerda de $B$ é a multiplicação e a ação à direita de $A$ é dada por $f \cdot \varphi:=\psi \cdot f$, onde $\psi(g)=\varphi\left(g^{-1}\right), \psi, \varphi \in A$ e $f \in C^{\infty}(M)$.

Queremos mostrar que

$$
C^{\infty}(M) \otimes_{C^{\infty}(M) \rtimes_{\alpha} G} C^{\infty}(M) \cong C^{\infty}(M / G)
$$

e

$$
C^{\infty}(M) \otimes_{C^{\infty}(M / G)} C^{\infty}(M) \cong C^{\infty}(M) \rtimes_{\alpha} G .
$$

Primeiramente observe que

$$
C^{\infty}(M) \otimes_{C^{\infty}(M) \rtimes_{\alpha} G} C^{\infty}(M) \cong C^{\infty}(M) \otimes_{\mathbb{C} \rtimes_{\alpha} G} \mathbb{C}
$$

através da aplicação dada por $f \otimes h \mapsto f h \otimes 1$. Dado $h \in G$ temos que $f h \otimes 1=f(1 \otimes h) \otimes 1=$ $f \otimes(1 \otimes h) 1=f \otimes 1$, para $f \otimes 1 \in C^{\infty}(M) \otimes_{\mathbb{C} \rtimes_{\alpha} G} \mathbb{C}$, logo podemos identificar $C^{\infty}(M) \otimes_{\mathbb{C} \rtimes_{\alpha} G} \mathbb{C}$ com o espaço de coinvariantes $H_{0}\left(G, C^{\infty}(M)\right)$. Como podemos identificar $H_{0}\left(G, C^{\infty}(M)\right)$ com $C^{\infty}(M / G)$ temos

$$
C^{\infty}(M) \otimes_{C^{\infty}(M) \rtimes_{\alpha} G} C^{\infty}(M) \cong C^{\infty}(M / G) .
$$

Como $M \times_{M / G} M \cong M \times G$ temos

$$
C^{\infty}(M) \otimes_{C^{\infty}(M / G)} C^{\infty}(M) \cong C^{\infty}(M) \otimes C^{\infty}(G)
$$

e $C^{\infty}(M) \otimes C^{\infty}(G) \cong C^{\infty}(M) \rtimes_{\alpha} G$ como $C^{\infty}(M) \rtimes_{\alpha} G$-módulo. Portanto

$$
C^{\infty}(M) \otimes_{C^{\infty}(M / G)} C^{\infty}(M) \cong C^{\infty}(M) \rtimes_{\alpha} G .
$$

\subsection{Hochschild-Kostant-Rosenberg}

O teorema de Hochschild-Kostant-Rosenberg identifica o anel de cohomologia de Hochschild da álgebra de funções suaves em uma variedade suave $M, H H^{*}\left(C^{\infty}(M)\right)$, com o anel dos campos multivetoriais em $M, \Gamma\left(\Lambda^{*} T M\right)$.

Teorema 3.7.1. Seja $M$ uma variedade suave compacta sem bordo. A aplicação $\Phi: \Gamma\left(\Lambda^{*} T M\right) \stackrel{\wedge}{\rightarrow}$ $H H^{*}\left(C^{\infty}(M)\right)$ dada por

$$
X_{1} \wedge \cdots \wedge X_{n} \mapsto \frac{1}{n !} \sum_{\sigma \in S_{n}} \operatorname{sgn}(\sigma) \widehat{X}_{\sigma}(1) \smile \cdots \smile \widehat{X}_{\sigma}(n),
$$

onde $\widehat{X}(f)=d f(X)$ para $f \in C^{\infty}(M)$ e $X \in \Gamma\left(\Lambda^{1} T M\right)$, é um isomorfismo de anéis graduados e leva o produto wedge ao produto cup e o colchete de Schouten ao colchete de Gerstenhaber. 
Demonstração. Do lema ए.2. segue que a aplicação $\Phi$ é uma bijeção.

Sejam $X_{1}, \ldots, X_{n} n$ campos de vetores em $M$. Temos que se $U \in H H^{u}\left(C^{\infty}(M)\right)$ e $V \in H H^{v}\left(C^{\infty}(M)\right)$ então $U \smile V=(-1)^{u v} V \smile U$, disto segue que

$$
\begin{aligned}
\frac{1}{n !} \sum_{\sigma \in S_{n}} \operatorname{sgn}(\sigma) \widehat{X}_{\sigma}(1) \smile \cdots \smile \widehat{X}_{\sigma}(n) & =\frac{1}{n !} \sum_{\sigma \in S_{n}} \operatorname{sgn}\left(\sigma^{-1}\right) \operatorname{sgn}(\sigma) \widehat{X}_{1} \smile \cdots \wedge \widehat{X}_{n} \\
& =\widehat{X}_{1} \smile \cdots \smile \widehat{X}_{n},
\end{aligned}
$$

portanto $\Phi$ preserva os produtos.

Da regra de Leibniz

$$
[A, B \wedge C]=B \wedge[A, C]+(-1)^{(a-1) b}[A, B] \wedge C
$$

e do fato que $\Phi$ preserva produtos segue que para verificar que $\Phi$ preserva os colchetes só precisamos mostrar que $\Phi([A, B])=\llbracket \widehat{A}, \widehat{B} \rrbracket$, para $A, B$ campos vetoriais em $M$.

Sejam

$$
A=\sum a_{i} \frac{\partial}{\partial x_{i}} \text { e } B=\sum b_{i} \frac{\partial}{\partial x_{i}}
$$

dois campos vetoriais em $M$. Temos que

$$
\Phi([A, B])(f)=\sum_{j} a_{j} \sum_{i} \frac{\partial b_{i}}{\partial x_{j}} \frac{\partial f}{\partial x_{i}}-\sum_{j} b_{j} \sum_{i} \frac{\partial a_{i}}{\partial x_{j}} \frac{\partial f}{\partial x_{i}}
$$

$\mathrm{e}$

$$
\llbracket \widehat{A}, \widehat{B} \rrbracket=\widehat{A} * \widehat{B}-\widehat{B} * \widehat{A}
$$

De

$$
\widehat{A} * \widehat{B}(f)=\widehat{A}\left(\sum_{i} b_{i} \frac{\partial f}{\partial x_{i}}\right)=\sum_{j} a_{j} \frac{\partial}{\partial x_{j}}\left(\sum_{i} b_{i} \frac{\partial f}{\partial x_{i}}\right)=\sum_{j} a_{j} \sum_{i} \frac{\partial b_{i}}{\partial x_{j}} \frac{\partial f}{\partial x_{i}}+\sum_{j} a_{j} \sum_{i} b_{i} \frac{\partial^{2} f}{\partial x_{j} x_{i}}
$$

$\mathrm{e}$

$$
\widehat{B} * \widehat{A}(f)=\sum_{j} b_{j} \sum_{i} \frac{\partial a_{i}}{\partial x_{j}} \frac{\partial f}{\partial x_{i}}+\sum_{j} b_{j} \sum_{i} a_{i} \frac{\partial^{2} f}{\partial x_{j} x_{i}}
$$

segue que

$$
\llbracket \widehat{A}, \widehat{B} \rrbracket(f)=\sum_{j} a_{j} \sum_{i} \frac{\partial b_{i}}{\partial x_{j}} \frac{\partial f}{\partial x_{i}}-\sum_{j} b_{j} \sum_{i} \frac{\partial a_{i}}{\partial x_{j}} \frac{\partial f}{\partial x_{i}}=\Phi([A, B])(f)
$$

Observação. Note que se considerarmos $C^{\infty}(M)$ como um espaço de Fréchet temos que os campos de multivetores que correspondem a uma aplicação contínua são campos suaves. Desta forma temos o seguinte isomorfismo

$$
H H_{\text {cont }}^{*}\left(C^{\infty}(M)\right) \stackrel{\sim}{\rightarrow} \Gamma^{\infty}\left(\Lambda^{*}(T M)\right) .
$$




\section{Capítulo 4}

\section{Geometria de Poisson Não Comutativa}

\subsection{Estruturas de Poisson em Álgebras}

No presente capítulo introduzimos os objetos de estudo principais deste trabalho, as estruturas de Poisson não comutativas. Os resultados principais do capítulo são os teoremas 4.3.3 e 4.4.2, os quais nos mostram que temos uma estrutura de Poisson não comutativa nos quocientes de variedades de Poisson e no toro não comutativo, respectivamente.

Do teorema 3.7. segue que podemos identificar um bivetor em uma variedade $M$ com uma classe de grau 2 da cohomologia de Hochschild de $C^{\infty}(M)$, também segue que se $\pi$ é um bivetor de Poisson e $\Pi$ é sua imagem pela aplicação $\Phi$ do teorema então $\llbracket \Pi, \Pi \rrbracket=0$.

Desta forma podemos identificar bivetores de Poisson em uma variedade $M$ com classes em $H H^{2}\left(C^{\infty}(M)\right)$ que satisfazem uma certa condição. Baseados neste fato fazemos a seguinte definição

Definição 4.1.1. Seja $A$ uma álgebra topológica localmente convexa. Uma estrutura de Poisson não comutativa em $A$ é um elemento $\Pi$ em $H H^{2}(A)$ que satisfaz

$$
\llbracket \Pi, \Pi \rrbracket=0 .
$$

Com essa definição podemos introduzir muitos dos objetos que vimos no Capítulo 1 em álgebras associativas.

Dada uma estrutura de Poisson não comutativa $\Pi$ em uma álgebra associativa $A$ definimos o campo Hamiltoniano associado a um elemento $c \in H H^{0}(A)$ como

$$
X_{c}=\llbracket \Pi, c \rrbracket \in H H^{1}(A) .
$$

Definimos um colchete de Poisson $H H^{0}(A)$ da seguinte forma

$$
\{c, e\}:=\llbracket X_{e}, c \rrbracket \in H H^{0}(A)
$$

Proposição 4.1.1. Sejam $c, e \in H H^{0}(A)$. Valem as seguintes propriedades

1. $\llbracket X_{c}, \Pi \rrbracket=0$

2. $\llbracket X_{c}, X_{e} \rrbracket=-X_{\{c, e\}}$

3. $H H^{0}(A)$ com o colchete definido anteriormente é uma álgebra de Poisson no sentido usual.

Demonstração. Pela identidade de Jacobi temos

$$
(-1)^{(2-1)(0-1)} \llbracket \Pi, \llbracket \Pi, c \rrbracket \rrbracket+(-1)^{(2-1)(2-1)} \llbracket \Pi, \llbracket c, \Pi \rrbracket \rrbracket+(-1)^{(0-1)(2-1)} \llbracket c, \llbracket \Pi, \Pi \rrbracket \rrbracket=0,
$$


$\log 0$

$$
-\llbracket \Pi, \llbracket \Pi, c \rrbracket \rrbracket-\llbracket \Pi, \llbracket c, \Pi \rrbracket \rrbracket=0 .
$$

Como $\llbracket c, \Pi \rrbracket=-(-1)^{(2-1)(0-1)} \llbracket \Pi, c \rrbracket=\llbracket \Pi, c \rrbracket$, temos

$$
\llbracket \Pi, \llbracket \Pi, c \rrbracket \rrbracket=0 \Rightarrow \llbracket X_{c}, \Pi \rrbracket=0 .
$$

Novamente pela identidade de Jacobi, temos

$$
(-1)^{(2-1)(0-1)} \llbracket \Pi, \llbracket X_{e}, c \rrbracket \rrbracket+(-1)^{(1-1)(2-1)} \llbracket X_{e}, \llbracket c, \Pi \rrbracket \rrbracket+(-1)^{(0-1)(1-1)} \llbracket c, \llbracket \Pi, X_{e} \rrbracket \rrbracket=0 .
$$

Como $\llbracket X_{e}, \Pi \rrbracket=0$, temos

$$
-\llbracket \Pi, \llbracket X_{e}, c \rrbracket \rrbracket+\llbracket X_{e}, \llbracket c, \Pi \rrbracket \rrbracket=0 \Rightarrow-\llbracket \Pi,\{e, c\} \rrbracket=\llbracket X_{e}, X_{c} \rrbracket \Rightarrow \llbracket X_{c}, X_{e} \rrbracket=-X_{\{c, e\}} .
$$

Com estas definições podemos falar de dinâmica Hamiltoniana não comutativa em álgebras associativas de forma análoga ao caso comutativo.

A seguir vamos dar alguns exemplos de álgebras de Poisson não comutativas.

Exemplo 4.1.1. Seja $A=C^{\infty}(M)$ a álgebra de funções de uma variedade de Poisson $M$. Pelo teorema 5.7. o bivetor de Poisson pode ser identificado com um elemento $\Pi \in H H^{2}(A)$ que nos dá uma estrutura de Poisson não comutativa em $A$.

Exemplo 4.1.2. Seja $M$ uma variedade de Poisson e tome $A=C^{\infty}(M) \otimes M_{k}(\mathbb{C})$. Podemos definir П,uma estrutura de Poisson não comutativa em $A$ da seguinte forma

$$
\Pi(f \otimes a, g \otimes b)=\{f, g\} \otimes a b .
$$

Exemplo 4.1.3. Nas seções 4.3 e 4.4 veremos mais dois exemplos, uma estrutura de Poisson não comutativa em um quociente de Poisson e no toro não comutativo, respectivamente.

\subsection{Cohomologia de Poisson}

Podemos definir cohomologia de Poisson para estruturas de Poisson não comutativas de forma muito similar ao caso clássico, usando o colchete de Gerstenhaber.

O seguinte lema nos permite definir um complexo associado a uma estrutura de Poisson não comutativa.

Lema 4.2.1. Seja $U \in H H^{u}(A)$, então

$$
\llbracket \Pi, \llbracket \Pi, U \rrbracket \rrbracket=0 .
$$

Demonstração. Segue diretamente da identidade de Jacobi e do fato que $\llbracket \Pi, \Pi \llbracket=0$, assim como no caso clássico.

Seja

$$
d_{\Pi}: H H^{i}(A) \rightarrow H H^{i+1}(A)
$$

a aplicação dada por

$$
d_{\Pi}(U)=\llbracket \Pi, U \rrbracket .
$$

Do lema 4.2.$]$ segue que $d_{\Pi} \circ d_{\Pi}=0$. Chamaremos a cohomologia do complexo $\left(H H^{*}(A), d_{\Pi}\right)$ de cohomologia de Poisson de $(A, \Pi)$ e vamos denotá-la por $H_{\Pi}^{*}(A)$.

Podemos interpretar a cohomologia de Poisson de uma álgebra de Poisson $(A, \Pi)$ de forma análoga à cohomologia de Poisson de variedades de Poisson. 
Definição 4.2.1. Uma deformação de Poisson de uma álgebra de Poisson $(A, \Pi)$ é uma série formal

$$
\Pi_{\lambda}=\sum_{i=0}^{\infty} \lambda^{i} \Pi_{i}
$$

tal que $\llbracket \Pi_{\lambda}, \Pi_{\lambda} \rrbracket=0$, onde $\Pi_{i} \in H H^{2}(A)$ para todo $i$ e $\Pi_{0}=\Pi$.

Temos

$$
\llbracket \Pi_{\lambda}, \Pi_{\lambda} \rrbracket=\sum_{j=0}^{\infty} \lambda^{j}\left(\sum_{i+j=n} \llbracket \Pi_{i}, \Pi_{j} \rrbracket\right) .
$$

Logo

$$
\begin{aligned}
\llbracket \Pi_{\lambda}, \Pi_{\lambda} \rrbracket=0 & \Longleftrightarrow \sum_{i+j=n} \llbracket \Pi_{i}, \Pi_{j} \rrbracket=0, \text { para todo } n \\
& \Longleftrightarrow 2 d_{\Pi} \Pi_{n}=-\sum_{i+j=k, i \geq 1, j \geq 1} \llbracket \Pi_{i}, \Pi_{j} \rrbracket, \text { para todo } n
\end{aligned}
$$

Da equação $\Pi_{\text {L. }}$ segue que $d_{\Pi}\left(\Pi_{1}\right)=0$ e, portanto, $\Pi_{1}$ é um cociclo em $H_{\Pi}^{2}(A)$ que chamaremos de infinitesimal da deformação $\Pi_{\lambda}$. Temos a seguinte proposição

Proposição 4.2.2. Dado $\Pi_{1} \in H H^{2}(A)$ tal que $d_{\Pi}\left(\Pi_{1}\right)=0$ a obstrução para existência de uma deformação de Poisson com infinitesimal $\pi_{1}$ é $H_{\Pi}^{3}(M)$. Isto é, se $H_{\Pi}^{3}(M)=0$ então existe uma deformação de Poisson com infinitesimal $\Pi_{1}$.

Demonstração. A demonstração é a mesma da proposição ए.5.2, basta apenas trocar o colchete de Schouten pelo colchete de Gerstenhaber.

Vamos mostrar a existência de uma sequência $\left\{\Pi_{n}\right\}_{n=2}^{\infty}$ que satisfaz a equação 4.$]$ por indução. Suponha que encontramos $\Pi_{2}, \ldots, \Pi_{k-1}$ que satifazem $\|_{\text {. }}$. Seja

$$
E_{k}=-\sum_{i+j=k, i \geq 1, j \geq 1} \llbracket \Pi_{i}, \Pi_{j} \rrbracket .
$$

Para mostrar que existe um $\Pi_{k}$ que satisfaz a equação $\mathbb{L}_{\text {. }}$ basta mostrar que $d_{\Pi}\left(E_{k}\right)=0$ pois $H_{\Pi}^{3}(M)=0$. Temos

$$
\begin{aligned}
d_{\Pi}\left(E_{k}\right) & =\sum_{i+j=k, i \geq 1, j \geq 1}\left(\llbracket \Pi_{i}, d_{\Pi} \Pi_{j} \rrbracket+\llbracket \Pi_{j}, d_{\Pi} \Pi_{i} \rrbracket\right) \\
& =2 \sum_{i+j=k, i \geq 1, j \geq 1} \llbracket \Pi_{j}, d_{\Pi} \Pi_{i} \rrbracket \\
& =-\sum_{i+j=k, i \geq 1, j \geq 1}\left(\sum_{\alpha+\beta=i, \alpha \geq 1, \beta \geq 1} \llbracket \Pi_{j}, \llbracket \Pi_{\alpha}, \Pi_{\beta} \rrbracket \rrbracket\right) \\
& =-\sum_{\alpha+\beta+j=k, \alpha \geq 1, \beta \geq 1, j \geq 1}^{\llbracket \Pi_{j}, \llbracket \Pi_{\alpha}, \Pi_{\beta} \rrbracket \rrbracket} \\
& =-\frac{1}{3} \sum_{\alpha+\beta+j=k, \alpha \geq 1, \beta \geq 1, j \geq 1}\left(\llbracket \Pi_{j}, \llbracket \Pi_{\alpha}, \Pi_{\beta} \rrbracket \rrbracket+\llbracket \Pi_{\alpha}, \llbracket \Pi_{\beta}, \Pi_{j} \rrbracket \rrbracket+\llbracket \Pi_{\beta}, \llbracket \Pi_{j}, \Pi_{\alpha} \rrbracket \rrbracket\right) \\
& =0 .
\end{aligned}
$$

Observação. Deformações de estruturas de Poisson aparecem em problemas de quantização. Veja (Tang, 20104). 


\subsection{Redução de Poisson}

Um dos principais motivos para nós introduzirmos estas estruturas de Poisson não comutativas é estudar a redução quando temos um quociente ruim. Como vimos no Capítulo 2 quando temos uma ação de um grupo de Lie $G$ em uma variedade suave $M$ devemos substituir o quociente usual pela álgebra $C_{c}^{\infty}\left(G, C_{c}^{\infty}(M)\right)$ com o produto

$$
(f \times g)(t)=\int_{G} f(s) \alpha_{s} g\left(s^{-1} t\right) d s .
$$

A partir de agora vamos assumir que $(M, \pi)$ é uma variedade de Poisson compacta e $G$ é um grupo de Lie que age em $M$ preservando a estrutura de Poisson. Seja $A=C_{c}^{\infty}\left(G, C^{\infty}(M)\right)$.

Temos a seguinte proposição sobre a álgebra $A$.

Proposição 4.3.1. O complexo bar associado à álgebra $A$ é exato.

Demonstração. Observe que podemos identificar o complexo bar com o seguinte complexo

$$
0 \longleftarrow C_{c}^{\infty}\left(G, C_{c}^{\infty}(M)\right) \overleftarrow{b} C_{c}^{\infty}\left(G \times G, C_{c}^{\infty}(M)\right) \overleftarrow{b} C_{c}^{\infty}\left(G^{3}, C_{c}^{\infty}(M)\right) \longleftarrow \cdots
$$

onde $b$ é dado por

$$
(b f)\left(g_{1}, \ldots, g_{n}\right)=\sum_{i=1}^{n}(-1)^{i-1} f\left(g_{1}, \ldots, g_{i}, g_{i}, \ldots, g_{n}\right),
$$

para $f \in C_{c}^{\infty}\left(G^{n+1}, C_{c}^{\infty}(M)\right)$.

Defina a aplicação $s: C_{c}^{\infty}\left(G^{n}, C_{c}^{\infty}(M)\right) \rightarrow C_{c}^{\infty}\left(G^{n+1}, C_{c}^{\infty}(M)\right)$ da seguinte maneira

$$
(s f)\left(g_{1}, \ldots, g_{n+1}\right)=f\left(g_{2}, \ldots, g_{n+1}\right) \text {. }
$$

Temos

$$
\begin{aligned}
b(s f)\left(g_{1}, \ldots, g_{n}\right) & =\sum_{i=1}^{n}(-1)^{i-1}(s f)\left(g_{1}, \ldots, g_{i}, g_{i}, \ldots, g_{n}\right) \\
& =f\left(g_{1}, \ldots, g_{n}\right)+\sum_{i=2}^{n}(-1)^{i-1} f\left(g_{2}, \ldots, g_{i}, g_{i}, \ldots, g_{n}\right)
\end{aligned}
$$

e

$$
s(b f)\left(g_{1}, \ldots, g_{n}\right)=(b f)\left(g_{2}, \ldots, g_{n}\right)=\sum_{i=2}^{n}(-1)^{i} f\left(g_{2}, \ldots, g_{i}, g_{i}, \ldots, g_{n}\right),
$$

$\operatorname{logo} s b+b s=1$ e portanto $s$ é uma contração do complexo. Concluímos assim que o complexo é exato e portanto o complexo bar associado à álgebra $A$ é exato.

Defina a seguinte aplicação bilinear contínua $\Pi: A \times A \rightarrow A$ por

$$
\Pi(f, g)(t)=\int_{G}\left\{f(s), \alpha_{s} g\left(s^{-1} t\right)\right\} d s
$$

onde $f, g \in A, t \in G$.

Proposição 4.3.2. П é um cociclo de Hochschild.

Demonstração. Temos

$$
\partial \Pi(f, g, h)=f \times \Pi(g, h)-\Pi(f \times g, h)+\Pi(f, g \times h)-\Pi(f, g) \times h
$$




$$
\begin{aligned}
f \times \Pi(g, h) & =\int_{G} \int_{G} f(s)\left\{\alpha_{s} g\left(s^{-1} u\right), \alpha_{u} h\left(u^{-1} t\right)\right\} d u d s \\
\Pi(f \times g, h) & =\int_{G} \int_{G} f(s)\left\{\alpha_{s} g\left(s^{-1} u\right), \alpha_{u} h\left(u^{-1} t\right)\right\} d u d s \\
& +\int_{G} \int_{G} \alpha_{s} g\left(s^{-1} u\right)\left\{f(s), \alpha_{u} h\left(u^{-1} t\right)\right\} d u d s \\
\Pi(f, g \times h) & =\int_{G} \int_{G}\left\{f(s), \alpha_{s} g\left(s^{-1} u\right)\right\} \alpha_{u} h\left(u^{-1} t\right) d u d s \\
& +\int_{G} \int_{G}\left\{f(s), \alpha_{u} h\left(u^{-1} t\right)\right\} \alpha_{s} g\left(s^{-1} u\right) d u d s \\
\Pi(f, g) \times h= & \int_{G} \int_{G}\left\{f(s), \alpha_{s} g\left(s^{-1} u\right)\right\} \alpha_{u} h\left(u^{-1} t\right) d u d s .
\end{aligned}
$$

Logo $\partial \Pi=0$.

Para este $\Pi$ definido anteriormente ser uma estrutura de Poisson em $A$ falta verificar que $\llbracket \Pi, \Pi \rrbracket=0$. Veremos que sob uma certa condição isto de fato acontece.

Como $\pi$ é uma estrutura de Poisson em $M$ temos que $[\pi, \pi]=0$, pelo Teorema $5.7 .-1$ segue que $D: C^{\infty}(M) \otimes C^{\infty}(M) \rightarrow C^{\infty}(M)$ dada por $D(f \otimes g)=\pi(\mathrm{d} f \wedge \mathrm{d} g)=\{f, g\}$ satisfaz $\llbracket D, D \rrbracket=0$ em cohomologia. Isto é, existe uma 2-cocadeia de Hochschild $B_{2}$ tal que $\llbracket D, D \rrbracket=\partial B_{2}$.

Teorema 4.3.3. Seja $(M, \pi)$ uma variedade de Poisson, $G$ um grupo de Lie que age em $M$ preservando a estrutura de Poisson e $D: C^{\infty}(M) \otimes C^{\infty}(M) \rightarrow C^{\infty}(M)$ dada por $D(f \otimes g)=$ $\pi(d f \wedge d g)=\{f, g\}$. Seja $A=C_{c}^{\infty}\left(G, C^{\infty}(M)\right)$ e $\Pi: A \times A \rightarrow A$ a seguinte aplicação bilinear contínua

$$
\Pi(f, g)(t)=\int_{G}\left\{f(s), \alpha_{s} g\left(s^{-1} t\right)\right\} d s .
$$

Se existir uma 2-cocadeia $G$-invariante $B_{2}: C^{\infty}(M) \otimes C^{\infty}(M) \rightarrow C^{\infty}(M)$ tal que $\llbracket D, D \rrbracket=\partial B_{2}$ então $\llbracket \Pi, \Pi \rrbracket=0$. Isto é, sob esta condição $\Pi$ é uma estrutura de Poisson não comutativa em A.

Demonstração. Observe que

$$
\begin{aligned}
\llbracket D, D \rrbracket(f, g, h) & =2 D(D(f, g), h)-2 D(f, D(g, h)) \\
& =2\{\{f, g\}, h\}-2\{f,\{g, h\}\} .
\end{aligned}
$$

Temos

$$
\begin{aligned}
\llbracket \Pi, \Pi \rrbracket(f, g, h)(t)= & 2 \Pi(\Pi(f, g), h)(t)-2 \Pi(f, \Pi(g, h))(t) \\
= & \int_{G} \int_{G} 2\left\{\left\{f(s), \alpha_{s} g\left(s^{-1} u\right)\right\}, \alpha_{u} h\left(u^{-1} t\right)\right\} d s d u \\
& -\int_{G} \int_{G} 2\left\{f(s),\left\{\alpha_{s} g\left(s^{-1} u\right), \alpha_{u} h\left(u^{-1} t\right)\right\}\right\} d s d u \\
= & \int_{G} \int_{G} \llbracket D, D \rrbracket\left(f(s), \alpha_{s} g\left(s^{-1} u\right), \alpha_{u} h\left(u^{-1} t\right)\right) d s d u \\
= & \int_{G} \int_{G}\left(\partial B_{2}\right)\left(f(s), \alpha_{s} g\left(s^{-1} u\right), \alpha_{u} h\left(u^{-1} t\right)\right) d s d u
\end{aligned}
$$

Defina

$$
A_{2}(f, g)(t)=\int_{G} B_{2}\left(f(s), \alpha_{s} g\left(s^{-1} t\right)\right) d s
$$

Temos

$$
\left(\partial A_{2}\right)(f, g, h)(t)=\left(f \times A_{2}(g, h)\right)(t)-\left(A_{2}(f \times g, h)\right)(t)+\left(A_{2}(f, g \times h)\right)(t)-\left(A_{2}(f, g) \times h\right)(t)
$$


e como $B_{2}$ é $G$-invariante segue que

$$
\begin{gathered}
\left(f \times A_{2}(g, h)\right)(t)=\int_{G} f(s) \alpha_{s} A_{2}(g, h)\left(s^{-1} t\right) d s=\int_{G} \int_{G} f(s) B_{2}\left(\alpha_{s} g(u), \alpha_{s u} h\left(u^{-1} s^{-1} t\right) d u d s\right. \\
A_{2}(f \times g, h)(t)=\int_{G} B_{2}(f \times g(u)) \alpha_{u} h\left(u^{-1} t\right) d u=\int_{G} \int_{G} B_{2}\left(f(s) \alpha_{s} g\left(s^{-1} u\right), \alpha_{u} h\left(u^{-1} t\right)\right) d u d s \\
A_{2}(f, g \times h)(t)=\int_{G} B_{2}\left(f(s), \alpha_{s}(g \times h)\left(s^{-1} t\right)\right) d s=\int_{G} \int_{G} B_{2}\left(f(s), \alpha_{s} g(u) \alpha_{u} h\left(u^{-1} s^{-1} t\right)\right) d u d s \\
\left(A_{2}(f, g) \times h\right)(t)=\int_{G} A_{2}(f, g)(u) \alpha_{u} h\left(u^{-1} t\right) d u=\int_{G} \int_{G} B_{2}\left(f(s), \alpha_{s} g\left(s^{-1} u\right)\right) \alpha_{u} h\left(u^{-1} t\right) d u d s .
\end{gathered}
$$

Como $d u$ é uma medida invariante à esquerda temos

$$
\begin{aligned}
\left(f \times A_{2}(g, h)\right)(t) & =\int_{G} \int_{G} f(s) B_{2}\left(\alpha_{s} g(u), \alpha_{s u} h\left(u^{-1} s^{-1} t\right) d u d s\right. \\
& =\int_{G} \int_{G} f(s) B_{2}\left(\alpha_{s} g\left(s^{-1} u\right), \alpha_{u} h\left(u^{-1} t\right) d u d s\right.
\end{aligned}
$$

e

$$
\begin{aligned}
A_{2}(f, g \times h)(t) & =\int_{G} \int_{G} B_{2}\left(f(s), \alpha_{s} g(u) \alpha_{u} h\left(u^{-1} s^{-1} t\right)\right) d u d s \\
& =\int_{G} \int_{G} B_{2}\left(f(s), \alpha_{s} g\left(s^{-1} u\right) \alpha_{u} h\left(u^{-1} t\right) d u d s .\right.
\end{aligned}
$$

Disto segue que

$$
\left(\partial A_{2}\right)(f, g, h)(t)=\int_{G} \int_{G}\left(\partial B_{2}\right)\left(f(s), \alpha_{s} g\left(s^{-1} u\right), \alpha_{u} h\left(u^{-1} t\right) d u d s\right.
$$

e portanto $\llbracket \Pi, \Pi \rrbracket=\partial A_{2}$.

Se $G$ é um grupo compacto, sempre existe uma 2-cocadeia $\hat{B}_{2} G$-invariante tal que $\llbracket D, D \rrbracket=$ $\partial \hat{B}_{2}$. De fato, sabemos que existe uma 2-cadeia $B_{2}$ tal que $\llbracket D, D \rrbracket=\partial B_{2}$. Seja $\tilde{B}_{2}: C^{\infty}(M) \otimes$ $C^{\infty}(M) \rightarrow C^{\infty}(M)$ dada por

$$
\tilde{B}_{2}(f, g)=\int_{G} \alpha_{u} B_{2}\left(\alpha_{u^{-1}} f, \alpha_{u^{-1}} g\right) d u
$$

onde $d u$ é a medida unimodular em $G$ que satisfaz $\int_{G} d u=1$. Temos

$$
\begin{aligned}
\alpha_{s} \tilde{B}_{2}(f, g) & =\int_{G} \alpha_{s u} B_{2}\left(\alpha_{u^{-1}} f, \alpha_{u^{-1}} g\right) d u \\
& =\int_{G} \alpha_{u} B_{2}\left(\alpha_{u^{-1} s} f, \alpha_{u^{-1} s} g\right) d u
\end{aligned}
$$

$\mathrm{e}$

$$
\tilde{B}_{2}\left(\alpha_{s} f, \alpha_{s} g\right)=\int_{G} \alpha_{u} B_{2}\left(\alpha_{u^{-1} s} f, \alpha_{u^{-1} s} g\right) d u .
$$

Logo $\tilde{B}_{2}$ é $G$-invariante.

Como $D$ é $G$-invariante temos

$$
\begin{aligned}
\partial\left(\tilde{B}_{2}\right)(f, g, h)= & \int_{G} f \alpha_{u} B_{2}\left(\alpha_{u^{-1}} g, \alpha_{u^{-1}} h\right) d u-\int_{G} \alpha_{u} B_{2}\left(\alpha_{u^{-1}} f g, \alpha_{u^{-1}} h\right) d u \\
& +\int_{G} \alpha_{u} B_{2}\left(\alpha_{u^{-1}} f, \alpha_{u^{-1}} g h\right) d u-\int_{G} \alpha_{u} B_{2}\left(\alpha_{u^{-1}} f, \alpha_{u^{-1}} g\right) h d u \\
= & \int_{G} \alpha_{u}\left(\partial B_{2}\right)\left(\alpha_{u^{-1}} f, \alpha_{u^{-1}} g, \alpha_{u^{-1}} h\right) d u \\
= & \int_{G} \alpha_{u} \llbracket D, D \rrbracket\left(\alpha_{u^{-1}} f, \alpha_{u^{-1}} g, \alpha_{u^{-1}} h\right) d u \\
= & \int_{G} \llbracket D, D \rrbracket(f, g, h) d u \\
= & \llbracket D, D \rrbracket(f, g, h) .
\end{aligned}
$$


Logo $\llbracket D, D \rrbracket=\partial\left(\tilde{B}_{2}\right)$.

Desta forma concluimos

Corolário 4.3.4. Se G é compacto então П é uma estrutura de Poisson em A.

Demonstração. Segue diretamente da proposição 4.3 .3 que $\Pi$ é uma estrutura de Poisson não comutativa em $A$.

No caso em que $M / G$ é suave, temos o seguinte

Corolário 4.3.5. Se a ação de $G$ em $M$ for livre e própria, II é uma estrutura de Poisson não comutativa em A.

Demonstração. Sob estas condições temos que $M / G$ é uma variedade de Poisson com a estrutura $\hat{D}$ induzida pela estrutura $D$ em $M$. Logo existe uma 2-cadeia $\hat{B}_{2}: C_{c}^{\infty}(M / G) \otimes C_{c}^{\infty}(M / G) \rightarrow$ $C_{c}^{\infty}(M / G)$, tal que $\llbracket \hat{D}, \hat{D} \rrbracket=\partial \hat{B}_{2}$.

Seja $\left\{U_{\alpha}\right\}_{\alpha \in \Lambda}$ um atlas em $M / G$ que trivializa o fibrado principal $\pi: M \rightarrow M / G$, isto é, para cada $\alpha \in \Lambda$ temos $\pi^{-1}\left(U_{\alpha}\right) \cong U_{\alpha} \times G$. Suponha que $\left\{\varphi_{\alpha}\right\}_{\alpha \in \Lambda}$ seja uma partição da unidade subordinada à cobertura $\left\{U_{\alpha}\right\}_{\alpha \in \Lambda}$. Para cada $\alpha \in \Lambda$ defina $B_{2}^{\alpha}: C^{\infty}(M) \otimes C^{\infty}(M) \rightarrow C^{\infty}(M)$ da seguinte maneira. Para cada $x \in \pi^{-1}\left(U_{\alpha}\right)$ seja $(y, s) \in U_{\alpha} \times G$ o elemento que corresponde a $x$. Defina

$$
B_{2}^{\alpha}(f, g)(x)=\hat{B}_{2}\left(f \circ \xi_{s}^{\alpha}, g \circ \xi_{s}^{\alpha}\right)(\pi(x)), \quad \forall f, g \in C_{c}^{\infty}(M),
$$

onde $\xi_{s}^{\alpha}: U_{\alpha} \rightarrow \pi^{-1}\left(U_{\alpha}\right) \cong U_{\alpha} \times G$, para cada $s \in G$, é a seção dada por $\xi_{s}^{\alpha}(z)=(z, s)$, $\forall z \in U_{\alpha}$. Observe que $f \circ \xi_{s}^{\alpha}, g \circ \xi_{s}^{\alpha}$ são funções suaves em $U_{\alpha}$. Usaremos a mesma notação para denotar uma extensão suave de $f \circ \xi_{s}^{\alpha}, g \circ \xi_{s}^{\alpha}$ para $C_{c}^{\infty}(M / G)$.

Defina $B_{2}$ da seguinte forma

$$
B_{2}(f, g)(x)=\sum_{\alpha \in \Lambda} \varphi_{\alpha}(\pi(x)) B_{2}^{\alpha}(f, g)(x), \quad \forall f, g \in C_{c}^{\infty}(M) .
$$

Como $B_{2}^{\alpha}$ é $G$-invariante segue pela construção anterior que $B_{2}$ é $G$-invariante e

$$
\begin{aligned}
\left(\partial B_{2}^{\alpha}\right)(f, g, h)(x)= & f(x) \hat{B}_{2}\left(g \circ \xi_{s}^{\alpha}, h \circ \xi_{s}^{\alpha}\right)(\pi(x))-\hat{B}_{2}\left(f g \circ \xi_{s}^{\alpha}, h \circ \xi_{s}^{\alpha}\right)(\pi(x)) \\
& +\hat{B}_{2}\left(f \circ \xi_{s}^{\alpha}, g h \circ \xi_{s}^{\alpha}\right)(\pi(x))-\hat{B}_{2}\left(f \circ \xi_{s}^{\alpha}, g \circ \xi_{s}^{\alpha}\right)(\pi(x)) h(x) \\
= & \partial \hat{B}_{2}\left(f \circ \xi_{s}^{\alpha}, g \circ \xi_{s}^{\alpha}, h \circ \xi_{s}^{\alpha}\right)(\pi(x)) \\
= & \llbracket \hat{D}, \hat{D} \rrbracket \hat{B}_{2}\left(f \circ \xi_{s}^{\alpha}, g \circ \xi_{s}^{\alpha}, h \circ \xi_{s}^{\alpha}\right)(\pi(x)) \\
= & \llbracket D, D \rrbracket(f, g, h)(x) .
\end{aligned}
$$

Portanto,

$$
\begin{aligned}
\left(\partial B_{2}\right)(f, g, h)(x) & =\sum_{\alpha \in \Lambda} \varphi_{\alpha}(\pi(x))\left(\partial B_{2}^{\alpha}\right)(f, g, h)(x) \\
& =\sum_{\alpha \in \Lambda} \varphi_{\alpha}(\pi(x)) \llbracket D, D \rrbracket(f, g, h)(x) \\
& =\llbracket D, D \rrbracket(f, g, h)(x) .
\end{aligned}
$$

Logo $\llbracket D, D \rrbracket=\partial B_{2}$ e como $\partial B_{2}$ é $G$-invariante a conclusão segue da proposição 4.3 .3 .3 .

No que segue iremos assumir que $G$ é um grupo compacto com uma ação de Poisson de $G$ em uma variedade de Poisson compacta $M$ e que tal ação é livre.

De acordo com o corolário 4.3 .5 a álgebra de produto cruzado suave $A=C^{\infty}(M) \rtimes_{\alpha} G$ possui uma estrutura de Poisson não comutativa dada pelo cociclo $\Pi \in H H^{2}(A)$ do Teorema 4.3.31. Por outro lado, neste caso temos que $M / G$ é uma variedade de Poisson, como vimos no capítulo 四. 
Temos que, como álgebras topológicas, $A$ é Morita equivalente a $C^{\infty}(M / G)$, onde a equivalência é dada pelo bimódulo $C^{\infty}(M)$. Portanto seus grupos de cohomologia de Hochschild são isomorfos, mais do que isso eles são isomorfos a $\Gamma^{\infty}\left(\Lambda^{*}(M / G)\right)$, o espaço de campos de multivetores suaves em $M / G$.

O teorema a seguir de (Xn1, [1994) diz que $\Pi \in H H^{2}(A)$ corresponde ao tensor de Poisson reduzido $\hat{\pi} \in H H^{2}\left(C^{\infty}(M / G)\right)$ sob o isomorfismo induzido pela equivalência de Morita.

Teorema 4.3.6. Sob o isomorfismo entre $H H^{2}(A)$ e $H H^{2}\left(C^{\infty}(M / G)\right)$ induzido pela equivalência de Morita, a estrutura de Poisson $\Pi$ em A corresponde ao tensor de Poisson reduzido $\hat{\pi}$, considerado como um elemento em $\mathrm{HH}^{2}\left(C^{\infty}(M / G)\right)$.

Demonstração. A demonstração pode ser vista em ( $\left(\mathrm{X}_{\mathbf{1}}\right.$, , [994) $)$ e se baseia no fato de podermos calcular tudo localmente pois $\Pi$ e $\hat{\pi}$ são seções de um fibrado vetorial. Desta forma podemos assumir que $M \cong M / G \times G$ e com isso a álgebra de produto cruzado assume uma forma mais simples e assim podemos fazer os cálculos necessários.

Desta forma temos que os dois processos de redução, clássico e o não comutativo, se equivalem de certo modo.

\subsection{Toro não comutativo}

Um dos exemplos mais importantes em geometria não comutativa é o toro não comutativo. Ele surge no estudo da folheação de Kronecker do 2-toro usual. Nesta seção introduziremos uma estrutura de Poisson não comutativa no toro não comutativo e computar sua cohomologia de Poisson.

Denotaremos por $S\left(Z^{2}\right)$ o espaço das sequências $\left(a_{n, m}\right)$ que decaem rapidamente, isto é, $(|n|+|m|)^{q}\left|a_{n, m}\right|$ é limitada para cada $q \in \mathbb{N}$.

Definição 4.4.1. Para cada $\theta \notin \mathbb{Q}$, seja $\lambda=\exp (2 \pi i \theta)$. Dado $\theta \notin \mathbb{Q}$, o toro não comutativo associado a $\theta$ é a álgebra $A_{\theta}$ que é definida da seguinte forma. $A_{\theta}$ é a álgebra cujo elemento genérico é a soma formal

$$
\sum_{n, m} a_{n, m} U_{1}^{n} U_{2}^{m}
$$

$\operatorname{com}\left(a_{n, m}\right) \in S\left(Z^{2}\right)$ e o produto dado pela relação

$$
U_{2} U_{1}=\lambda U_{1} U_{2}
$$

$A_{\theta}$ possui uma topologia gerada pelas seminormas

$$
P_{q}(a)=\sup _{n, m \in \mathbb{N}}\left((1+|n|+|m|)^{q}\left|a_{n, m}\right|\right)
$$

que a torna uma álgebra localmente convexa.

Podemos definir duas derivações $\delta_{1}$ e $\delta_{2}$ em $A_{\theta}$ da seguinte maneira

$$
\delta_{1}\left(U_{1}^{n} U_{2}^{m}\right)=2 \pi i n U_{1}^{n} U_{2}^{m}
$$

e

$$
\delta_{2}\left(U_{1}^{n} U_{2}^{m}\right)=2 \pi i m U_{1}^{n} U_{2}^{m} .
$$

Lema 4.4.1. $\llbracket \delta_{1}, \delta_{2} \rrbracket=0, \llbracket \delta_{1}, \delta_{1} \rrbracket=0 e \llbracket \delta_{2}, \delta_{2} \rrbracket=0$.

Demonstração.

$$
\llbracket \delta_{1}, \delta_{2} \rrbracket\left(U_{1}^{n} U_{2}^{m}\right)=\delta_{1} \delta_{2}\left(U_{1}^{n} U_{2}^{m}\right)-\delta_{2} \delta_{1}\left(U_{1}^{n} U_{2}^{m}\right)=-4 \pi m n\left(U_{1}^{n} U_{2}^{m}\right)+4 \pi m n\left(U_{1}^{n} U_{2}^{m}\right)=0 .
$$

As outras identidades seguem da super-comutatividade do colchete de Gerstenhaber. 
Teorema 4.4.2. Seja $\Pi=\delta_{1} \wedge \delta_{2} \in H H^{2}\left(A_{\theta}\right)$. Temos que $\Pi$ é uma estrutura de Poisson em $A_{\theta}$.

Demonstração. Utilizando a regra de Leibniz temos

$$
\begin{aligned}
\llbracket \delta_{1} \wedge \delta_{2}, \delta_{1} \wedge \delta_{2} \rrbracket & =-\llbracket \delta_{1} \wedge \delta_{2}, \delta_{1} \rrbracket \wedge \delta_{2}+\delta_{1} \wedge \llbracket \delta_{2}, \delta_{1} \wedge \delta_{2} \rrbracket \\
& =\llbracket \delta_{1}, \delta_{1} \rrbracket \wedge \delta_{2} \wedge \delta_{2}+\delta_{1} \wedge \llbracket \delta_{1}, \delta_{2} \rrbracket \wedge \delta_{2}-\delta_{1} \wedge \llbracket \delta_{2}, \delta_{1} \rrbracket \wedge \delta_{2}-\delta_{1} \wedge \delta_{1} \wedge \llbracket \delta_{2}, \delta_{2} \rrbracket \\
& =0 .
\end{aligned}
$$

Agora vamos calcular a cohomologia de Hochschild topológica de $A_{\theta}$. Para isso vamos precisar de uma resolução projetiva topológica de $A_{\theta}$.

Lema 4.4.3. Seja $\Omega=\Omega_{0} \otimes \Omega_{1} \otimes \Omega_{2}$ a álgebra exterior gerada pelo conjunto $\left\{e_{1}, e_{2}\right\}$. Então

$$
A_{\theta} \overleftarrow{\epsilon} A_{\theta}^{e} \widehat{\otimes} \Omega_{0}\left(=M_{0}\right) \overleftarrow{b_{1}} A_{\theta}^{e} \widehat{\otimes} \Omega_{1}\left(=M_{1}\right) \overleftarrow{b_{2}} A_{\theta}^{e} \widehat{\otimes} \Omega_{2}\left(=M_{2}\right) \longleftarrow 0
$$

é uma resolução projetiva topológica de $A_{\theta}$, onde $\epsilon(a \otimes b)=a b$,

$$
b_{1}\left(1 \otimes e_{j}\right)=1 \otimes U_{j}-U_{j} \otimes 1
$$

$e$

$$
b_{2}\left(1 \otimes\left(e_{1} \wedge e_{2}\right)\right)=\left(U_{2} \otimes 1-\lambda \otimes U_{2}\right) \otimes e_{1}-\left(\lambda U_{1} \otimes 1-1 \otimes U_{1}\right) \otimes e_{2} .
$$

Demonstração. Para $\nu=\left(n_{1}, n_{2}\right) \in Z^{2}$, seja $U^{\nu}=U_{1}^{n_{1}} U_{2}^{n_{2}} \in A_{\theta}, X^{\nu}=U^{\nu} \otimes 1 \in A_{\theta}^{e} \mathrm{e}$ $Y^{\nu}=1 \otimes U^{\nu} \in A_{\theta}^{e}$. Então $X^{\nu}$ e $Y^{\nu^{\prime}}$ comutam para todo $\nu, \nu^{\prime}$ e cada elemento de $A_{\theta}^{e}$ é da forma $a=\sum a_{\nu, \nu^{\prime}} X^{\nu} Y^{\nu^{\prime}}$, onde a sequência $\left(a_{\nu, \nu^{\prime}}\right)$ é um elemento arbitrário de $S\left(Z^{4}\right)$.

Temos $X^{\nu} X^{\nu^{\prime}}=\lambda^{n_{2} n_{1}^{\prime}} X^{\nu+\nu^{\prime}}$ e $Y^{\nu} Y^{\nu^{\prime}}=\lambda^{n_{2}^{\prime} n_{1}} Y^{\nu+\nu^{\prime}}$.

Vamos verificar que $\operatorname{ker} \epsilon=\operatorname{Im} b_{1}$. A inclusão $\operatorname{Im} b_{1} \subset \operatorname{ker} \epsilon$ segue diretamente da definição. Para $a=\sum a_{\nu, \nu^{\prime}} X^{\nu} Y^{\nu^{\prime}}, \epsilon(a)=0$ implica que vale $\sum a_{\nu, \nu^{\prime}} X^{\nu} X^{\nu^{\prime}}=0$ e portanto $a=\sum a_{\nu, \nu^{\prime}} X^{\nu}\left(Y^{\nu^{\prime}}-X^{\nu^{\prime}}\right)$. Usando a igualdade

$$
\begin{aligned}
\left(1 \otimes U_{2}^{n_{2}}\right)\left(1 \otimes U_{1}^{n_{1}}\right)-\left(U_{1}^{n_{1}} \otimes 1\right)\left(U_{2}^{n_{2}} \otimes 1\right) & =\left(1 \otimes U_{2}^{n_{2}}\right)\left(\sum_{j=0}^{n_{1}-1} U_{1}^{j} \otimes U_{1}^{n_{1}-1-j}\right)\left(1 \otimes U_{1}-U_{1} \otimes 1\right) \\
& +\left(U_{1}^{n_{1}} \otimes 1\right)\left(\sum_{j=0}^{n_{2}-1} U_{2}^{j} \otimes U_{2}^{n_{2}-1-j}\right)\left(1 \otimes U_{2}-U_{2} \otimes 1\right)
\end{aligned}
$$

e observando que

$$
X^{\nu}\left(Y^{\nu^{\prime}}-X^{\nu^{\prime}}\right)=X^{\nu}\left(1 \otimes U_{1}^{n_{1}^{\prime}} U_{2}^{n_{2}^{\prime}}-U_{1}^{n_{1}^{\prime}} U_{2}^{n_{2}^{\prime}} \otimes 1\right)=X^{\nu}\left(\left(1 \otimes U_{2}^{n_{2}^{\prime}}\right)\left(1 \otimes U_{1}^{n_{1}^{\prime}}\right)-\left(U_{1}^{n_{1}^{\prime}} \otimes 1\right)\left(U_{2}^{n_{2}^{\prime}} \otimes 1\right)\right),
$$

temos que ker $\epsilon$ é gerado por $1 \otimes U_{1}-U_{1} \otimes 1$ e $1 \otimes U_{2}-U_{2} \otimes 1$ e portanto é igual a $\operatorname{Im} b_{1}$.

Checamos diretamente que $b_{1} b_{2}=0$. Dado $a=a_{1} \otimes e_{1}-a_{2} \otimes e_{2} \in \operatorname{ker} b_{1}$, temos $a_{1}\left(1 \otimes U_{1}-\right.$ $\left.U_{1} \otimes 1\right)=a_{2}\left(1 \otimes U_{2}-U_{2} \otimes 1\right)$. Observe que para $a$ pertencer a $\operatorname{Im} b_{2}$ basta encontrar $y \in A_{\theta}^{e}$ que satisfaz $a_{1}=y\left(U_{2} \otimes 1-\lambda \otimes U_{2}\right)$.

Tomando $Z=\lambda U_{2}^{-1} \otimes U_{2}$, mostramos que $a_{1}\left(\sum_{-\infty}^{\infty} Z^{k}\right)=0$ usando a identidade

$$
\begin{aligned}
a_{1}\left(\sum_{-\infty}^{\infty} Z^{k}\right)\left(1 \otimes U_{1}-U_{1} \otimes 1\right) & =a_{1}\left(1 \otimes U_{1}-U_{1} \otimes 1\right)\left(\sum_{-\infty}^{\infty}\left(U_{2}^{-1} \otimes U_{2}\right)^{k}\right) \\
& =a_{2}\left(1 \otimes U_{2}-U_{2} \otimes 1\right)\left(\sum_{-\infty}^{\infty}\left(U_{2}^{-1} \otimes U_{2}\right)^{k}\right) \\
& =0 .
\end{aligned}
$$


Escrevendo $a_{1}=\sum x_{k} Z^{k}$, onde $x_{k}$ é um elemento da subálgebra de $A_{\theta}^{e}$ gerada pelos elementos $1 \otimes U_{1}, U_{1} \otimes 1$ e $U_{2} \otimes 1$, temos $\sum x_{k}=0$ e portanto

$$
a_{1}=\sum x_{k}\left(Z^{k}-1\right)=\sum x_{k}\left(\sum_{j=0}^{k-1} Z^{j}\right)(Z-1)=y_{1}(Z-1) .
$$

Segue que

$$
a_{1}=-y_{1}\left(U_{2}^{-1} \otimes 1\right)\left(U_{2} \otimes 1\right)(Z-1)=y_{1}\left(U_{2}^{-1} \otimes 1\right)\left(U_{2} \otimes 1-\lambda \otimes U_{2}\right) .
$$

Por fim basta apenas verificar que $b_{2}$ é injetora, porém isto segue diretamente da definição.

Usando a resolução anterior podemos calcular $H H^{*}\left(A_{\theta}\right)$. Diremos a seguir que $\theta$ satisfaz uma condição Diofantina se a sequência $\left(\left|1-\lambda^{n}\right|^{-1}\right)$ é $O\left(n^{k}\right)$ para algum $k$ (lembre que dizemos que uma sequência $\left(a_{n}\right)$ é $O\left(n^{k}\right)$ se existe $n_{0}$ tal que para cada $n \geq n_{0}$ vale $\left|a_{n}\right| \leq M n^{k}$ para alguma constante $M)$.

Proposição 4.4.4. Seja $\theta \notin \mathbb{Q}$.

- $H H^{0}\left(A_{\theta}\right)=\mathbb{C}$ e $H H^{i}\left(A_{\theta}\right)=0$, para $i \geq 3$.

- Se $\theta$ satisfaz uma condição Diofantina, então $H H^{1}\left(A_{\theta}\right)$ tem dimensão 2 e $H H^{2}\left(A_{\theta}\right)$ tem dimensão 1.

- Se $\theta$ não satisfaz uma condição Diofantina, então $H H^{1}\left(A_{\theta}\right)$ e $H H^{2}\left(A_{\theta}\right)$ possuem dimensão infinita.

Demonstração. Primeiramente observe que é óbvio que $H H^{i}\left(A_{\theta}\right)=0$ para $i \geq 0$ devido ao tamanho da resolução de $A_{\theta}$ que temos.

Queremos calcular o cohomologia do complexo

$$
0 \longrightarrow \operatorname{Hom}_{A^{e}}\left(M_{0}, A_{\theta}\right) \stackrel{b_{1}^{*}}{\longrightarrow} \operatorname{Hom}_{A^{e}}\left(M_{1}, A_{\theta}\right) \stackrel{b_{2}^{*}}{\longrightarrow} \operatorname{Hom}_{A^{e}}\left(M_{2}, A_{\theta}\right) \longrightarrow 0 .
$$

Sabemos que

$$
\begin{gathered}
\operatorname{Hom}_{A^{e}}\left(M_{0}, A_{\theta}\right) \cong \operatorname{Hom}\left(\Omega_{0}, A_{\theta}\right) \cong \Omega_{0}^{*} \otimes A_{\theta} \cong \mathbb{C}^{*} \otimes A_{\theta} \cong A_{\theta} ; \\
\operatorname{Hom}_{A^{e}}\left(M_{1}, A_{\theta}\right) \cong \operatorname{Hom}\left(\Omega_{1}, A_{\theta}\right) \cong \Omega_{0}^{*} \otimes A_{\theta} \cong\left(\mathbb{C}^{2}\right)^{*} \otimes A_{\theta} \cong A_{\theta} \oplus A_{\theta} ;
\end{gathered}
$$

e

$$
\operatorname{Hom}_{A^{e}}\left(M_{2}, A_{\theta}\right) \cong \operatorname{Hom}\left(\Omega_{2}, A_{\theta}\right) \cong \Omega_{2}^{*} \otimes A_{\theta} \cong \mathbb{C}^{*} \otimes A_{\theta} \cong A_{\theta} .
$$

Sob estes isomorfismos o complexo 4.2 fica

$$
0 \longrightarrow A_{\theta} \stackrel{\alpha_{1}}{\longrightarrow} A_{\theta} \oplus A_{\theta} \stackrel{\alpha_{2}}{\longrightarrow} A_{\theta} \longrightarrow 0 .
$$

Calculamos diretamente que $\alpha_{1}(a)=\left(a U_{1}-U_{1} a, a U_{2}-U_{2} a\right)$ e $\alpha_{2}\left(a_{1}, a_{2}\right)=U_{2} a_{1}-\lambda a_{1} U_{2}-$ $\lambda U_{1} a_{2}+a_{2} U_{1}$. Temos que $\mathbb{C} \subset \operatorname{ker} \alpha_{1}$ e dado $a \in A_{\theta}$ tal que $a \notin \mathbb{C}$ temos

$$
\alpha_{1}(a)=0 \Longleftrightarrow a U_{1}=U_{1} a \text { e } a U_{2}=U_{2} a \Longleftrightarrow \lambda U_{1} a=U_{1} a \text { e } \lambda U_{2} a=U_{2} a \Longleftrightarrow \lambda=1 .
$$

Porém, como $\theta \notin \mathbb{Q}$ temos que $\lambda \neq 1$ e portanto $\operatorname{ker}\left(\alpha_{1}\right)=\mathbb{C}$. Logo $H H^{0}\left(A_{\theta}\right)=\mathbb{C}$.

A partir de agora usaremos a notação $f_{i}=\sum a_{m, n}^{i} U_{1}^{m} U_{2}^{n}$, para $i=1,2$, e $f=\sum a_{m, n} U_{1}^{m} U_{2}^{n}$. Suponha que $\left(f_{1}, f_{2}\right) \in \operatorname{ker}\left(\alpha_{2}\right)$, temos $U_{2} f_{1}-\lambda f_{1} U_{2}=\lambda U_{1} f_{2}-f_{2} U_{1}$ e disto segue que

$$
\left(1-\lambda^{n_{2}}\right) a_{n_{1}+1, n_{2}}^{1}=\left(\lambda^{n_{1}}-1\right) a_{n_{1}, n_{2}+1}^{2} \quad \forall n_{1}, n_{2} .
$$


Se $\alpha_{1}(f)=\left(f_{1}, f_{2}\right)$ temos que $f$ satisfaz $f U_{1}-U_{1} f=f_{1}$ e $f U_{2}-U_{2} f=f_{2}$ e portanto

$$
\left(1-\lambda^{n_{2}}\right) a_{n_{1}, n_{2}}=a_{n_{1}+1, n_{2}}^{1} \text { e }\left(\lambda^{n_{1}}-1\right) a_{n_{1}, n_{2}}=a_{n_{1}, n_{2}+1}^{2} .
$$

Essas condições são compatíveis se, e somente se, $a_{1,0}^{1}=a_{0,1}^{2}=0$.

Logo se $\left(f_{1}, f_{2}\right) \in \operatorname{ker}\left(\alpha_{2}\right)$ e satisfaz as condições anteriores podemos definir $f$ utilizando a identidade $\left(1-\lambda^{n_{2}}\right) a_{n_{1}, n_{2}}=a_{n_{1}+1, n_{2}}^{1}$. Se $\theta$ satisfaz uma condição Diofantina a sequência $a_{n, m}$ definida de fato está em $A_{\theta}$ e portanto $H H^{1}\left(A_{\theta}\right)=\mathbb{C}^{2}$. Se $\theta$ não satisfaz uma condição Diofantina podemos encontrar infinitas sequências $a_{n, m}^{1}$ que decrescem rapidamente enquanto $a_{n, m}=a_{n, m}^{1}(1-\lambda)^{-1}$ não decresce rapidamente, neste caso $H H^{1}\left(A_{\theta}\right)$ tem dimensão infinita. A demonstração para $H H^{2}$ é similar.

Com isso podemos calcular a cohomologia de Poisson de $\left(A_{\theta}, \Pi\right)$.

Proposição 4.4.5. • $H_{\Pi}^{0}\left(A_{\theta}\right)=\mathbb{C}$ e $H_{\Pi}^{i}\left(A_{\theta}\right)=0$ para $i \geq 3$.

- Se $\theta$ satisfaz uma condição Diofantina, então

$$
H_{\Pi}^{1}\left(A_{\theta}\right)=\mathbb{C} \oplus \mathbb{C} \text { e } H_{\Pi}^{2}\left(A_{\theta}\right)=\mathbb{C} .
$$

- Se $\theta$ não satisfaz uma condição Diofantina, então $H_{\Pi}^{1}\left(A_{\theta}\right)$ e $H_{\Pi}^{2}\left(A_{\theta}\right)$ possuem dimensão infinita.

Observe que a estrutura de Poisson em $A_{\theta}$ está definida utilizando a resolução usual $M_{k}^{\prime}=$ $A_{\theta}^{e} \widehat{\otimes} A_{\theta}^{\otimes k}$. Para ver com o que esta estrutura de Poisson se corresponde na resolução $M_{k}=$ $A_{\theta}^{e} \widehat{\otimes} \Omega_{k}$ precisamos comparar as duas resoluções, isto é, precisamos encontrar dois morfismos de complexos de $A^{e}$-módulos $h: M \rightarrow M^{\prime}$ e $k: M^{\prime} \rightarrow M$ que são a identidade em grau 0.

Lema 4.4.6. As aplicações de complexos de $A^{e}$-módulos $h: M \rightarrow M^{\prime}$ e $k: M^{\prime} \rightarrow M$ descritas acima podem ser escolhidas de forma que

$$
\begin{gathered}
h_{1}\left(1 \otimes e_{j}\right)=-1 \otimes U_{j}, j=1,2 ; \\
h_{2}\left(1 \otimes\left(e_{1} \wedge e_{2}\right)\right)=\lambda 1 \otimes U_{1} \otimes U_{2}-1 \otimes U_{2} \otimes U_{1} ;
\end{gathered}
$$

$e$

$$
k_{1}\left(1 \otimes U_{j}\right)=-1 \otimes e_{j}, j=1,2 .
$$

Demonstração. Lembre que

$$
\begin{aligned}
b_{n}^{\prime}\left(1 \otimes a_{1} \otimes \cdots \otimes a_{n}\right) & =\left(a_{1} \otimes 1\right) \otimes\left(a_{2} \otimes \cdots \otimes a_{n}\right) \\
& +\sum_{j=1}^{n-1}(-1)^{j} 1 \otimes a_{1} \otimes \cdots \otimes a_{j} a_{j+1} \otimes \cdots \otimes a_{n} \\
& +(-1)^{n}\left(1 \otimes a_{n}\right) \otimes\left(a_{1} \otimes \cdots \otimes a_{n-1}\right) .
\end{aligned}
$$

A aplicação $h_{1}$ é determinada por $h_{1}\left(1 \otimes e_{j}\right)$ e deve satisfazer

$$
b_{1}^{\prime} h_{1}\left(1 \otimes e_{j}\right)=b_{1}\left(1 \otimes e_{j}\right)=1 \otimes U_{j}-U_{j} \otimes 1,
$$

portanto podemos tomar $h_{1}\left(1 \otimes e_{j}\right)=-1 \otimes U_{j}$.

Temos que $h_{2}$ é determinada por $h_{2}\left(1 \otimes\left(e_{1} \wedge e_{2}\right)\right)$ e satisfaz

$$
\begin{aligned}
b_{2}^{\prime} h_{2}\left(1 \otimes\left(e_{1} \wedge e_{2}\right)\right) & =h_{1} b_{2}\left(1 \otimes\left(e_{1} \wedge e_{2}\right)\right) \\
& =h_{1}\left(\left(U_{2} \otimes 1-\lambda \otimes U_{2}\right) \otimes e_{1}-\left(\lambda U_{1} \otimes 1-1 \otimes U_{1}\right) \otimes e_{2}\right) \\
& =-\left(U_{2} \otimes 1-\lambda \otimes U_{2}\right)\left(1 \otimes U_{1}\right)+\left(\lambda U_{1} \otimes 1-1 \otimes U_{1}\right)\left(1 \otimes U_{2}\right) .
\end{aligned}
$$


Logo podemos tomar $h_{2}\left(1 \otimes\left(e_{1} \wedge e_{2}\right)\right)=\lambda 1 \otimes U_{1} \otimes U_{2}-1 \otimes U_{2} \otimes U_{1}$.

Podemos escolher $k_{1}$ de forma análoga. Temos que $k_{1}$ é determinado pelo valor de $k_{1}\left(1 \otimes U_{j}\right)$ e deve satisfazer

$$
b_{1} k_{1}\left(1 \otimes U_{j}\right)=b_{1}^{\prime}\left(1 \otimes U_{j}\right)=U_{j} \otimes 1-1 \otimes U_{j},
$$

portanto podemos tomar $k_{1}\left(1 \otimes U_{j}\right)=-1 \otimes e_{j}$.

Usando o complexo 4.3 temos:

Lema 4.4.7. Para cada $\left(a_{1}, a_{2}\right) \in \operatorname{ker} \alpha_{2} / \operatorname{Im} \alpha_{1}=H H^{1}\left(A_{\theta}\right)$, temos que $d_{\Pi}\left(a_{1}, a_{2}\right)$ é a classe do elemento $a \in H H^{2}\left(A^{\theta}\right)=A_{\theta} / \operatorname{Im} \alpha_{2}$ dado por

$$
a=2 \pi i \lambda\left[\left(2 \pi i a_{1}-\delta_{1} a_{1}\right) U_{2}+U_{1}\left(2 \pi i a_{2}-\delta_{2} a_{2}\right)\right]
$$

Demonstração. Temos as identificações $\left(a_{1}, a_{2}\right) \mapsto T \mapsto \tilde{\delta} \mapsto \delta$, onde $\left(a_{1}, a_{2}\right) \in A_{\theta} \oplus A_{\theta}, T \in$ $\operatorname{Hom}_{A^{e}}\left(A^{e} \otimes \Omega_{1}, A_{\theta}\right), \tilde{\delta} \in \operatorname{Hom}_{A^{e}}\left(A^{e} \otimes A_{\theta}, A_{\theta}\right)$ e $\delta \in \operatorname{Hom}\left(A_{\theta}, A_{\theta}\right)$. Estas aplicações estão relacionadas da seguinte maneira: $T\left(1 \otimes e_{i}\right)=a_{i}, \delta\left(U_{i}\right)=\tilde{\delta}\left(1 \otimes U_{i}\right)$ e $\tilde{\delta}=T \circ k_{1}$. Sob essas identificações temos

$$
\begin{aligned}
d_{\Pi}\left(a_{1}, a_{2}\right) & =d_{\Pi}(T)\left(1 \otimes\left(e_{1} \wedge e_{2}\right)\right) \\
& =d_{\Pi}(\tilde{\delta})\left(h_{2}\left(1 \otimes\left(e_{1} \wedge e_{2}\right)\right)\right) \\
& =d_{\Pi}(\tilde{\delta})\left(\lambda 1 \otimes U_{1} \otimes U_{2}-1 \otimes U_{2} \otimes U_{1}\right) \\
& =\lambda d_{\pi}(\delta)\left(U_{1}, U_{2}\right)-d_{\Pi}\left(U_{2}, U_{1}\right) \\
& =\lambda \llbracket \delta_{1} \wedge \delta_{2}, \delta \rrbracket\left(U_{1}, U_{2}\right)-\llbracket \delta_{1} \wedge \delta_{2}, \delta \rrbracket\left(U_{2}, U_{1}\right) \\
& =-\lambda\left(\llbracket \delta, \delta_{1} \rrbracket \wedge \delta_{2}\left(U_{1}, U_{2}\right)+\delta_{1} \wedge \llbracket \delta, \delta_{2} \rrbracket\left(U_{1}, U_{2}\right)\right) \\
& =-\lambda\left[\left(\delta \delta_{1} U_{1}-\delta_{1} \delta U_{1}\right)\left(\delta_{2} U_{2}\right)+\left(\delta_{1} U_{1}\right)\left(\delta \delta_{2} U_{2}-\delta_{2} \delta U_{2}\right)\right] \\
& =-\lambda\left[\left(2 \pi i \delta U_{1}-\delta_{1} \delta U_{1}\right)\left(2 \pi i U_{2}\right)+\left(2 \pi i U_{1}\right)\left(2 \pi i \delta U_{2}-\delta_{2} \delta U_{2}\right)\right]
\end{aligned}
$$

e

$$
\delta U_{i}=\tilde{\delta}\left(1 \otimes U_{i}\right)=T \circ k_{1}\left(1 \otimes U_{i}\right)=T\left(-1 \otimes e_{i}\right)=-a_{i} .
$$

Portanto

$$
d_{\Pi}\left(a_{1}, a_{2}\right)=2 \pi i \lambda\left[\left(2 \pi i a_{1}-\delta_{1} a_{1}\right) U_{2}+U_{1}\left(2 \pi i a_{2}-\delta_{2} a_{2}\right)\right] .
$$

Demonstração da proposição t4.4.5. Note que os dois primeiros itens da proposição segue diretamente do cálculo da cohomologia de Hochschild de $A_{\theta}$.

Perceba que $H_{\Pi}^{2}\left(A_{\theta}\right)=A_{\theta} /\left(\operatorname{Im} \alpha_{2}+\mathrm{d}_{\Pi}\left(\operatorname{ker} \alpha_{2}\right)\right)$. Seja

$$
a^{i}=\sum_{n, m} a_{n, m}^{i} U_{1}^{n} U_{2}^{m}, \quad i=1,2 .
$$

Então,

$$
d_{\Pi}\left(a^{1}, a^{2}\right)=\sum_{n, m} 4 \pi^{2} \lambda\left(a_{n, m-1}^{1}(n-1)+a_{n-1, m}^{2}(m-1)\right) U_{1}^{n} U_{2}^{m} .
$$

Dado $b \in\left(\operatorname{Im} \alpha_{2}+\mathrm{d}_{\Pi}\left(\operatorname{ker} \alpha_{2}\right)\right)$, assuma que $b=\sum_{n, m} b_{n, m} U_{1}^{n} U_{2}^{m}$. Então $b_{n, m}$ pode ser escrito da seguinte forma

$$
\begin{aligned}
b_{n, m} & =\tilde{a}_{n, m-1}^{1}\left(\lambda^{n}-\lambda\right)-\tilde{a}_{n-1, m}^{2}\left(\lambda-\lambda^{m}\right) \\
& +a_{n, m-1}^{1}(n-1)-a_{n-1, m}^{2}(m-1),
\end{aligned}
$$


para algum $\left(\tilde{a}^{1}, \tilde{a}^{2}\right) \in A_{\theta} \oplus A_{\theta}$ e $\left(a^{1}, a^{2}\right) \in \operatorname{ker} \alpha_{2}$. De $\alpha_{2}\left(a^{1}, a^{2}\right)=0$ segue que

$$
a_{n, m-1}^{1}\left(\lambda^{n}-\lambda\right)=a_{n-1, m}^{2}\left(\lambda-\lambda^{m}\right) .
$$

Tomando $m=1$ temos $a_{n, 0}^{1}\left(\lambda^{n}-\lambda\right)=0$. Portanto, $a_{n, 0}^{1}=0$ se $n \neq 1$. Tomando $m=1$ temos $b_{n, 1}=\tilde{a}_{n, 0}^{1}\left(\lambda^{n}-\lambda\right)$, para $n \neq 1$.

Logo uma condição necessária para $b \in\left(\operatorname{Im} \alpha_{2}+\mathrm{d}_{\Pi}\left(\operatorname{ker} \alpha_{2}\right)\right)$ é que $b_{n, 1}\left(\lambda^{n}-\lambda\right)^{-1}$ decresce rapidamente. Como $\theta$ não satisfaz uma condição Diofantina, podemos encontrar um número infinito de sequências $\left(b_{n, m}\right)$ com a propriedade que $\left(b_{n, 1}\right)$ decresce rapidamente e $b_{n, 1}\left(\lambda^{n}-\lambda\right)^{-1}$ não. Disto segue que $H^{2}\left(A_{\theta}\right)$ possui dimensão infinita. A demonstração para $H^{1}\left(A_{\theta}\right)$ é similar.

Seja

$$
\omega=U_{2}^{-1} U_{1}^{-1} \otimes U_{1} \otimes U_{2}-U_{1}^{-1} U_{2}^{-1} \otimes U_{2} \otimes U_{1}
$$

Temos

$\partial(\omega)=U_{2}^{-1} \otimes U_{2}-U_{2}^{-1} U_{1}^{-1} \otimes U_{1} U_{2}+U_{1}^{-1} \otimes U_{1}-U_{1}^{-1} \otimes U_{1}+U_{1}^{-1} U_{2}^{-1} \otimes U_{2} U_{1}-U_{2}^{-1} \otimes U_{2}=0$,

$\operatorname{logo} \omega$ é um 2-ciclo de Hochschild na álgebra $A_{\theta}$ e portanto define um elemento em $\mathrm{HH}_{2}\left(A_{\theta}\right)$.

A seguir vamos ver que este elemento $\omega$ em muitos aspectos se comporta como uma forma simplética em uma variedade.

Note que a contração induz uma aplicação $\omega^{b}: H H^{1}\left(A_{\theta}\right) \rightarrow H H_{1}\left(A_{\theta}\right)$ dada por

$$
\omega^{b}(\delta)=i_{\delta} \omega=-\delta\left(U_{2}\right) U_{2}^{-1} U_{1}^{-1} \otimes U_{1}+\delta\left(U_{1}\right) U_{1}^{-1} U_{2}^{-1} \otimes U_{2} .
$$

Temos o seguinte resultado análogo ao que temos em geometria simplética:

Proposição 4.4.8. Para $i=0,1,2$, as aplicações

$$
\begin{aligned}
\left(\omega^{b}\right)_{i}: H H^{i}\left(A_{\theta}\right) & \rightarrow H H_{2-i}\left(A_{\theta}\right) \\
U & \rightarrow i_{U} \omega
\end{aligned}
$$

são isomorfismos.

Para demonstrar essa proposição precisamos computar a homologia de Hochschild de $A_{\theta}$. Aplicando o funtor $A_{\theta} \widehat{\otimes}_{A_{\theta}^{e}}$. na resolução projetiva topológica $\left(M_{k}, b_{k}\right)$ que vimos anteriormente obtemos o complexo

$$
0 \longrightarrow A_{\theta} \widehat{\otimes}_{A_{\theta}^{e}} M_{2} \stackrel{\alpha_{2}}{\longrightarrow} A_{\theta} \widehat{\otimes}_{A_{\theta}^{e}} M_{1} \stackrel{\alpha_{1}}{\rightarrow} A_{\theta} \widehat{\otimes}_{A_{\theta}^{e}} M_{0} \longrightarrow 0
$$

$\operatorname{com} \alpha_{i}=1 \otimes b_{i}$.

Temos

$$
\alpha_{1}\left(a \otimes\left(1_{A^{e}} \otimes e_{i}\right)\right)=a \otimes\left(1 \otimes U_{i}-U_{i} \otimes 1\right)
$$

e

$$
\left.\alpha_{2}\left(a \otimes\left(1_{A^{e}} \otimes\left(e_{1} \wedge e_{2}\right)\right)\right)=a \otimes\left(U_{2} \otimes 1-\lambda \otimes U_{2}\right) \otimes e_{1}-\left(\lambda U_{1} \otimes 1-1 \otimes U_{1}\right) \otimes e_{2}\right) .
$$

Sob as identificações $A_{\theta} \widehat{\otimes}_{A^{e}} A^{e} \cong A_{\theta}$ dada por $a \otimes(b \otimes c) \mapsto c a b, A_{\theta} \otimes \Omega_{i} \cong A_{\theta}$, para $i=0,2$ e $A_{\theta} \otimes \Omega_{1} \cong A_{\theta} \oplus A_{\theta}$ o complexo anterior é mapeado para o complexo a seguir

$$
0 \longrightarrow A_{\theta} \stackrel{\beta_{2}}{\longrightarrow} A_{\theta} \oplus A_{\theta} \stackrel{\beta_{1}}{\longrightarrow} A_{\theta} \longrightarrow 0,
$$

onde

$$
\beta_{1}\left(a_{1}, a_{2}\right)=U_{1} a_{1}-a_{1} U_{1}+U_{2} a_{2}-a_{2} U_{2}
$$




$$
\beta_{2}(a)=\left(a U_{2}-\lambda U_{2} a, U_{1} a-\lambda a U_{1}\right) .
$$

Portanto a homologia de Hochschild $H H_{*}\left(A_{\theta}\right)$ pode ser computada como a homologia do complexo anterior.

Como as aplicações $\left(\omega^{b}\right)_{i}$ estão definidas utilizando a resolução bar $\left(M_{k}^{\prime}, b_{k}^{\prime}\right)$ precisaremos comparar as duas resoluções para escrever as aplicações $\left(\omega^{b}\right)_{i}$ com respeito à resolução $\left(M_{k}, b_{k}\right)$.

Denotando as aplicações correspondentes a $\left(\omega^{b}\right)_{i}$ na resolução $\left(M_{k}, b_{k}\right)$ por $h_{i}$ obtemos o seguinte diagrama

$$
\begin{aligned}
& 0 \longrightarrow A_{\theta} \stackrel{\alpha_{1}}{\longrightarrow} A_{\theta} \oplus A_{\theta} \stackrel{\alpha_{2}}{\longrightarrow} A_{\theta} \longrightarrow 0 \\
& \downarrow h_{0} \quad \downarrow h_{1} \quad \downarrow h_{2} \\
& 0 \longrightarrow A_{\theta} \stackrel{\beta_{2}}{\longrightarrow} A_{\theta} \stackrel{\downarrow}{\oplus} A_{\theta} \stackrel{\beta_{1}}{\longrightarrow} \stackrel{\downarrow}{A_{\theta}} \longrightarrow 0
\end{aligned}
$$

onde o complexo na linha superior é o complexo 4.3 que usamos para computar a cohomologia $H H^{*}\left(A_{\theta}\right)$.

Temos o seguinte lema:

Lema 4.4.9. $h_{0}, h_{1}$ e $h_{2}$ são isomorfismos. Além disso, temos

1. $h_{0}(a)=a U_{1}^{-1} U_{2}^{-1}$;

2. $h_{1}\left(a_{1}, a_{2}\right)=\left(a_{2} U_{2}^{-1} U_{1}^{-1},-a_{1} U_{1}^{-1} U_{2}^{-1}\right)$;

3. $h_{2}(a)=-a U_{1}^{-1} U_{2}^{-1}$.

E temos também que o diagrama 4.5 comuta.

Demonstração. Suponha que $\left(a_{1}, a_{2}\right) \in A_{\theta} \oplus A_{\theta}$ se corresponde com $\delta \in \operatorname{Hom}\left(A_{\theta}, A_{\theta}\right)$ sob as identificações usadas no 4.4.7. Então temos $\delta\left(U_{1}\right)=-a_{1}$ e $\delta\left(U_{2}\right)=-a_{2}$. Além disso temos que $h_{1}\left(a_{1}, a_{2}\right) \in A_{\theta} \oplus A_{\theta}$ corresponde a $\left(\omega^{b}\right)_{1}(\delta) \in A_{\theta} \widehat{\otimes} A_{\theta}$ pela definição de $h_{1}$. Como $\left(\omega^{b}\right)_{1}(\delta)=\delta\left(U_{2}\right) U_{2}^{-1} U_{1}^{-1} \otimes U_{1}-\delta\left(U_{1}\right) U_{1}^{-1} U_{2}^{-1} \otimes U_{2}$ corresponde a

$$
\delta\left(U_{2}\right) U_{2}^{-1} U_{1}^{-1} \otimes\left(1 \otimes U_{1}\right)-\delta\left(U_{1}\right) U_{1}^{-1} U_{2}^{-1} \otimes\left(1 \otimes U_{2}\right),
$$

temos

$$
\begin{aligned}
h\left(a_{1}, a_{2}\right) & =-\delta\left(U_{2}\right) U_{2}^{-1} U_{1}^{-1} \otimes\left(1 \otimes e_{1}\right)+\delta\left(U_{1}\right) U_{1}^{-1} U_{2}^{-1} \otimes\left(1 \otimes e_{2}\right) \\
& =-\delta\left(U_{2}\right) U_{2}^{-1} U_{1}^{-1} \otimes e_{1}+\delta\left(U_{1}\right) U_{1}^{-1} U_{2}^{-1} \otimes e_{2} \\
& =\left(-\delta\left(U_{2}\right) U_{2}^{-1} U_{1}^{-1}, \delta\left(U_{1}\right) U_{1}^{-1} U_{2}^{-1}\right) \\
& =\left(a_{2} U_{2}^{-1} U_{1}^{-1},-a_{1} U_{1}^{-1} U_{2}^{-1}\right) .
\end{aligned}
$$

Observe que $h_{1}$ possui uma inversa dada por

$$
\left(a_{1}, a_{2}\right) \mapsto\left(-a_{2} U_{2} U_{1}, a_{1} U_{1} U_{2}\right)
$$

portanto $h_{1}$ é um isomorfismo.

Podemos obter $h_{0}$ e $h_{2}$ de forma similar e segue que o diagrama comuta com um cálculo direto usando as fórmulas encontradas.

Demonstração da Proposição 4.4 .8 . A demonstração da proposição segue diretamente do lema anterior, isto é, do fato das aplicações $h_{i}$ serem isomorfismos.

Seja $d: H H_{n}(A) \rightarrow H H_{n+1}(A)$ a aplicação definida a nível de cadeias por

$$
d\left(a_{0} \otimes \cdots \otimes a_{n}\right)=\sum_{\sigma \in S_{n}}(\operatorname{sgn} \sigma)\left(1 \otimes a_{\sigma(1)} \otimes \cdots \otimes a_{\sigma(n)}-(-1)^{n+1} a_{\sigma(1)} \otimes \cdots \otimes a_{\sigma(n)} \otimes 1\right) .
$$


Lema 4.4.10. Vale $d\left(U_{i}^{-1} \otimes U_{i}\right)=0$, para $i=1,2$.

Demonstração. Temos

$$
\partial\left(U_{i}^{-1} \otimes U_{i} \otimes 1 \otimes 1\right)=1 \otimes 1 \otimes 1-U_{i}^{-1} \otimes U_{i} \otimes 1+U_{i}^{-1} \otimes U_{i} \otimes 1-U_{i}^{-1} \otimes U_{i} \otimes 1,
$$

da mesma forma temos $\partial\left(U_{i} \otimes U_{i}^{-1} \otimes 1 \otimes 1\right)=1 \otimes 1 \otimes 1-U_{i} \otimes U_{i}^{-1} \otimes 1$. Logo a nível de homologia temos

$$
U_{i} \otimes U_{i}^{-1} \otimes 1-U_{i}^{-1} \otimes U_{i} \otimes 1=0
$$

Da mesma maneira temos

$$
\partial\left(U_{i}^{-1} \otimes U_{i} \otimes U_{i}^{-1} \otimes U_{i}\right)=1 \otimes U_{i}^{-1} \otimes U_{i}-U_{i}^{-1} \otimes 1 \otimes U_{i}+U_{i}^{-1} \otimes U_{i} \otimes 1-1 \otimes U_{i} \otimes U_{i}^{-1},
$$

e

$$
\partial\left(U_{i}^{-1} \otimes U_{i} \otimes U_{i}^{-1} \otimes U_{i}\right)=U_{i}^{-1} \otimes 1 \otimes U_{i}+U_{i} \otimes U_{i}^{-1} \otimes 1 .
$$

Portanto, a nível de homologia temos

$$
1 \otimes U_{i}^{-1} \otimes U_{i}-U_{i} \otimes U_{i}^{-1} \otimes 1+U_{i}^{-1} \otimes U_{i} \otimes 1-1 \otimes U_{i} \otimes U_{i}^{-1}=0 .
$$

Das equações anteriores temos $1 \otimes U_{i}^{-1} \otimes U_{i}-1 \otimes U_{i} \otimes U_{i}^{-1}=0$. Logo,

$$
d\left(U_{i}^{-1} \otimes U_{i}\right)=1 \otimes U_{i}^{-1} \otimes U_{i}-U_{i}^{-1} \otimes U_{i} \otimes 1-1 \otimes U_{i} \otimes U_{i}^{-1}+U_{i} \otimes U_{i}^{-1} \otimes 1=0 .
$$

Proposição 4.4.11. Temos as seguintes identidades

$$
L_{\delta_{1}} \omega=0 \quad \text { e } \quad L_{\delta_{2}} \omega=0 .
$$

Demonstração. Como $d \omega=0$, temos $L_{\delta_{1}}=\left(d i_{\delta_{1}}+i_{\text {delta }_{j}} d\right) \omega=d i_{\delta_{1}} \omega$. Por outro lado,

$$
\begin{aligned}
i_{\delta_{1}} \omega & =i_{\delta_{1}}\left(U_{2}^{-1} U_{1}^{-1} \otimes U_{1} \otimes U_{2}-U_{1}^{-1} U_{2}^{-1} \otimes U_{2} \otimes U_{1}\right) \\
& =\delta_{1}\left(U_{2}\right) U_{2}^{-1} U_{1}^{-1} \otimes U_{1}-\delta_{1}\left(U_{1}\right) U_{1}^{-1} U_{2}^{-1} \otimes U_{2} \\
& =-2 \pi i U_{2}^{-1} \otimes U_{2} .
\end{aligned}
$$

Logo, pelo lema 4.4.10, vale $L_{\delta_{1}}=-2 \pi i d\left(U_{2}^{-1} \otimes U_{2}\right)=0$. Da mesma forma obtemos $L_{\delta_{2}}=0$.

Observação. Apesar das similaridades que vimos agora entre $\omega$ e uma estrutura simplética, ainda assim temos que a cohomologia de Poisson não comutativa é diferente da cohomologia de de Rham não comutativa(cohomologia cíclica, ver (Connes, 1994)), diferente do fato que vimos no capítulo $\square$ que a cohomologia de Poisson de variedades simpléticas coincide com a cohomologia de de Rham.

\subsection{Outras Aplicações}

\subsubsection{Folheações}

Suponha que $(M, \omega)$ é uma variedade pré-simplética. Isto é, $M$ é uma variedade suave equipada com uma 2-forma $\omega$. Sabemos que $\omega$ define uma folheação em $M$ dada pelo seu kernel e também que à cada folheação podemos associar uma álgebra de convolução que pode ser pensada como a álgebra de funções do espaço quociente da folheação.

Em (Block \& Getzler, 19.92) é introduzida uma estrutura de Poisson não comutativa nesta álgebra de convolução associada à uma variedade pré-simplética $(M, \omega)$. Um exemplo de variedade pré-simplética é dado por variedades de contato, neste caso temos que ker $\omega$ tem dimensão 1 e a álgebra de convolução é uma álgebra de produto cruzado onde a ação é dada por um fluxo. 


\subsubsection{Orbifolds}

Suponha que um grupo finito $G$ age em uma variedade suave $M$. Sabemos que quando esta ação não é livre o espaço $M / G$ não é uma variedade mas sim um orbifold, assim como substituto da álgebra de funções no quociente tomamos o produto cruzado $C^{\infty}(M) \rtimes G$.

Em (Halbout \& Tang, 2UIV) é calculado o colchete de Gerstenhaber na cohomologia de Hochschild $H H^{*}\left(C^{\infty}(M) \rtimes G\right)$ neste caso em que o grupo $G$ é finito. Com isso é feita uma descrição de todas as estruturas de Poisson não comutativas em $C^{\infty}(M) \rtimes G$ no caso em que $M$ é uma variedade simplética.

\subsubsection{Dinâmica Hamiltoniana}

Em (Kordyukov, 2010) é discutida a noção de fluxo Hamiltoniano no espaço de folhas de uma folheação transversalmente simplética de uma variedade compacta $M$ e é construída uma classe de campos Hamiltonianos. 


\section{Referências Bibliográficas}

Bass, H. (1968). Algebraic K-theory (1st ed.). W. A. Benjamin.

Block, J., \& Getzler, E. (1992). Quantization of Foliations. Proceedings of the XXth International Conference on Differential Geometric Methods in Physics (New York, 1991), 471-487.

Block, J., \& Getzler, E. (1994). Equivariant cyclic homology and equivariant differential forms. Ann. Sci. Éc. Norm. Supér. (4), 27(4), 493-527. doi: 10.24033/asens.1699

Connes, A. (1994). Noncommutative Geometry. Academic Press.

Dufour, J.-P., \& Zung, N. T. (2005). Poisson Structures and Their Normal Forms (1st ed.). Birkhäuser Basel.

Gerstenhaber, M. (1963). The cohomology structure of an associative ring. Ann. of Math. (2), 78, 267-288. Retrieved from https://doi.org/10.2307/1970343 doi: 10.2307/1970343

Halbout, G., \& Tang, X. (2010). Noncommutative Poisson Structures on Orbifolds. Transactions of the American Mathematical Society, 362(5), 2249-2277. Retrieved from http:// www.jstor.org/stable/25677784

Khalkhali, M. (2009). Basic Noncommutative Geometry. European Mathematical Society.

Kordyukov, Y. A. (2010). Noncommutative Hamiltonian dynamics on foliated manifolds. J. Fixed Point Theory Appl., 7.

Renault, J. (1980). A groupoid approach to $C^{*}$ - algebras (1st ed.).

Tang, X. (2004, 06). Deformation Quantization of Pseudo Symplectic(Poisson) Groupoids. Geometric and Functional Analysis, 16. doi: 10.1007/s00039-006-0567-6

Weibel, C. A. (1994). An Introduction to Homological Algebra. Cambridge University Press.

Williams, D. P. (2006). Crossed Products of $C^{*}$-algebras. Book Draft. Retrieved from https:// math.dartmouth.edu/ dana/cpcsa/draft-31Jan06.pdf

Witherspoon, S. (2010). An Introduction to Hochschild Cohomology. Book Draft. Retrieved from http://www.math.tamu.edu/ sjw/pub/HH-18May2018.pdf

Xu, P. (1994). Noncommutative Poisson Algebras. American Journal of Mathematics, 116(1), 101-125. Retrieved from http://www.jstor.org/stable/2374983 